\title{
Energy levels and transition probabilities for boron-like Fe $\mathrm{XxII}^{\star, \star \star}$
}

\author{
V. Jonauskas ${ }^{1,2}$, P. Bogdanovich ${ }^{2}$, F. P. Keenan ${ }^{1}$, R. Kisielius ${ }^{1,2}$, M. E. Foord ${ }^{3}$, R. F. Heeter ${ }^{3}$, S. J. Rose ${ }^{4}$, \\ G. J. Ferland ${ }^{5}$, and P. H. Norrington ${ }^{6}$
}

1 Department of Physics and Astronomy, The Queen's University of Belfast, Belfast BT7 1NN, Northern Ireland, UK e-mail: V.Jonauskas@qub.ac.uk

2 Institute of Theoretical Physics and Astronomy of Vilnius University, A. Goštauto 12, 01108 Vilnius, Lithuania

3 University of California, Lawrence Livermore National Laboratory, Livermore, CA 94551, USA

4 Department of Physics, Clarendon Laboratory, Parks Road, Oxford OX1 3PU, UK

5 Department of Physics, University of Kentucky, Lexington, KY 40506, USA

${ }^{6}$ Department of Applied Mathematics and Theoretical Physics, The Queen's University of Belfast, Belfast BT7 1NN, Northern Ireland, UK

Received 6 January 2006 / Accepted 17 May 2006

\section{ABSTRACT}

\begin{abstract}
The Multiconfiguration Dirac-Fock method is used to calculate the energies of the 407 lowest levels in Fe XXII. These results are cross-checked using a suite of codes which employ the configuration interaction method on the basis set of transformed radial orbitals with variable parameters, and takes into account relativistic corrections in the Breit-Pauli approximation. Transition probabilities, oscillator and line strengths are presented for electric dipole (E1), electric quadrupole (E2) and magnetic dipole (M1) transitions among these levels. The total radiative transition probabilities, as well as the five largest values from each level be it of E1, M1, E2, M2, or E3 type, are also provided. Finally, the results are compared with data compiled by NIST.
\end{abstract}

Key words. atomic data

\section{Introduction}

Physical processes and conditions in astrophysical sources can be understood through the use of theoretical models to analyse high resolution spectral observations. In particular, X-ray spectra from iron L-shell ions are prominent candidates for astrophysics plasma diagnostics, as their emission lines are observed in the wavelength range from 6-18 $\AA$, covered with large effective areas by X-ray telescopes on board the space observatories Chandra and XMM-Newton. Behar et al. (2001) identified Fe XXII lines in a spectrum of the Capella binary system obtained by the High Energy Transmission Grating Spectrometer on board Chandra, while Kinkhabwala et al. (2002) observed Fe XXII in the Seyfert 2 galaxy NGC 1068. To reliably analyse such spectra requires an accurate knowledge of wavelengths and radiative transition rates, obtained from calculations or experiment.

Numerous observations of boron-like iron have been previously reported in the X-ray spectra of the Sun (Doschek et al. 1973, 1981; Phillips et al. 1982; Fawcett et al. 1987; Phillips et al. 1996; McKenzie et al. 1985). Wargelin et al. (1998) have presented an analysis of the density sensitivity of Fe XXII L-shell lines in plasma obtained in the Princeton Large Torus tokamak. They analysed the intensity ratios of $4 d_{5 / 2}-2 p_{3 / 2}$ and $4 \mathrm{~d}_{3 / 2}-2 \mathrm{p}_{1 / 2}$ lines using the HULLAC atomic physics package (Bar-Shalom et al. 2001). Recently, Chen et al. (2004)

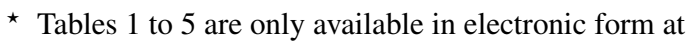
http://www . edpsciences.org

$\star \star$ Tables 6 to 8 are only available in electronic form at the CDS via anonymous ftp to cdsarc.u-strasbg.fr $(130.79 .128 .5)$ or via http://cdsweb.u-strasbg.fr/cgi-bin/qcat?J/A+A/455/1157
}

used a radiative-collisional model to predict the density of a plasma produced using the Electron Beam Ion Trap at Lawrence Livermore National Laboratory. They showed that the line ratios of the $2 p-3 d$ transitions in boron-like iron are sensitive to the electron density in the range $n_{\mathrm{e}}=$ $10^{13}-10^{15} \mathrm{~cm}^{-3}$. Earlier, electron-excitation rate calculations for 45 fine-structure levels, using the Breit-Pauli formulation of the R-matrix method, were performed by Zhang \& Pradhan (1997). Badnell et al. (2001) used the AUTOSTRUCTURE code (Badnell $1986,1997)$ to generate radial orbitals and atomic structure for 204-level close-coupling calculations in Fe XXII. A large set of energy levels is presented in the CHIANTI database at http://wwwsolar.nrl.navy .mil/chianti.html.

The aim of the present paper is extend our series of calculations for iron ions (Jonauskas et al. 2004a,b, 2005), by providing highly reliable energy levels and radiative transition rates for Fe XXII up to the $n=5$ complex. We note that in photoionized plasmas these high-lying levels will not in general be populated via electron impact (although collisional redistribution among the levels may play a role), but rather by a range of processes including recombination and charge transfer (Savin 2001). Transitions in Fe XXII from high-lying levels having 5d electrons were observed by Fawcett et al. (1987) in a solar flare spectrum. Wargelin et al. (1998) identified transitions from $2 s^{2} 4 d^{1}, 2 s^{1} 2 p^{1} 4 p^{1}, 2 s^{1} 2 p^{1} 5 p^{1}, 2 s^{2} 5 d^{1}, 2 s^{1} 2 p^{1} 5 d^{1}$ and $2 s^{2} 6 d^{1}$ configurations which are populated in a radiativecollisional model by electron collisions from metastable state.

In the present paper calculations are performed using the multiconfiguration Dirac-Fock (MCDF) approach. Energies of the lowest 407 levels, plus E1, E2 and M1 type radiative transition probabilities, as well as line and oscillator strengths among 
the levels of Fe XXII, are provided. The calculated results are compared with data compiled by NIST, as well as results obtained from other codes. The agreement between the length and velocity forms of electric transition operators is checked for the strongest transitions as an additional measure of the accuracy for the data obtained. The total radiative probabilities and five strongest probabilities required for calculating branching ratios of levels are presented as well.

\section{Method of calculation}

Results were obtained using the multiconfiguration DiracFock (MCDF) method employed in the GRASP code of Grant et al. (1980) and Parpia et al. (1996) (http://www.am.qub.ac.uk/DARC). To cross-check our MCDF data, we used a code developed by Bogdanovich \& Karpuškienè (1999), which adopts the configuration interaction (CI) method on the basis of transformed radial orbitals (TROs) with variable parameters, including relativistic effects in the Breit-Pauli approximation (CITRO).

\subsection{MCDF approach}

In the MCDF approach, intermediate coupling wavefunctions $\Psi_{\gamma}(J)$ are constructed by using an expansion of the form:

$\Psi_{\gamma}(J)=\sum_{\alpha} c_{\gamma}(\alpha J) \Phi(\alpha J)$

where configuration state functions (CSFs) $\Phi(\alpha J)$ are expressed as antisymmetrized products of two-component orbitals

$\phi(r)=\frac{1}{r}\left(\begin{array}{rr}P_{n l j}(r) & \chi_{l j m}(\hat{r}) \\ \mathrm{i} Q_{n \bar{l} j}(r) & \chi_{\bar{l} j m}(\hat{r})\end{array}\right)$.

Here $P_{n l j}(r)$ and $Q_{n \bar{l} j}(r)$ are the large and small radial components of one-electron wavefunctions and $\chi_{l j m}(\hat{r})$ are the two component Pauli spherical spinors. Two-component orbitals are obtained as the self-consistent solutions of the Dirac-Fock equations systematically increasing CI basis. Direct and indirect relativistic effects, when the contraction of inner orbitals leads to more effective screening of the nucleus for valence orbitals, are included in the relativistic wavefunctions by solving MCDF equations.

The configuration mixing coefficients $c_{\gamma}$ are obtained through diagonalization of the Dirac-Coulomb-Breit Hamiltonian:

$H^{\mathrm{DF}}=\sum_{i} h_{i}^{\mathrm{D}}+\sum_{i<j} h_{i j}^{\mathrm{e}}+\sum_{i<j} h_{i j}^{\mathrm{trans}}$,

where $h_{i}^{\mathrm{D}}$ is one-electron Dirac Hamiltonian, $h_{i j}^{\mathrm{e}}$ is the instantaneous Coulomb repulsion. The effects of the transverse interaction $h_{i j}^{\text {trans }}$ that corresponds to the Breit interaction:

$h_{i j}^{\text {Breit }}=-\frac{\alpha_{i} \cdot \alpha_{j}}{2 r_{i j}}-\frac{\left(\alpha_{i} \cdot r_{i j}\right)\left(\alpha_{j} \cdot r_{i j}\right)}{2 r_{i j}^{3}}$

in the low-frequency limit were evaluated in the first order of perturbation expansion. QED corrections, which include vacuum polarization and self-energy (known as the Lamb shift), are considered in the first order of perturbation theory.

One-electron excitations from the $2 \mathrm{~s}$ and $2 \mathrm{p}$ orbitals of the $1 \mathrm{~s}^{2} 2 \mathrm{~s}^{2} 2 \mathrm{p}^{1}, 1 \mathrm{~s}^{2} 2 \mathrm{~s}^{1} 2 \mathrm{p}^{2}$ and $1 \mathrm{~s}^{2} 2 \mathrm{p}^{3}$ configurations up to the $8 \mathrm{k}$ orbital, as well as two- and three-electron excitations from orbitals with $n=2$ to all possible combinations of two or three electrons in the shells up to $n=3$, were employed to generate one-electron wavefunctions as a basis set for CSFs in the MCDF method. Additionally, to extend the CI basis and obtain higher accuracy, an additional 14 configurations with electrons in $n=4$ are included, namely: $1 s^{2} 2 s^{1} 4 s^{2}, 1 s^{2} 2 s^{1} 4 p^{2}, 1 s^{2} 2 s^{1} 4 d^{2}, 1 s^{2} 2 s^{1} 4 f^{2}, 1 s^{2} 2 p^{1} 4 s^{2}$, $1 \mathrm{~s}^{2} 2 \mathrm{p}^{1} 4 \mathrm{p}^{2}, 1 \mathrm{~s}^{2} 2 \mathrm{p}^{1} 4 \mathrm{~d}^{2}, 1 \mathrm{~s}^{2} 2 \mathrm{p}^{1} 4 \mathrm{f}^{2}, 1 \mathrm{~s}^{2} 2 \mathrm{~s}^{1} 3 \mathrm{p}^{1} 4 \mathrm{p}^{1}, 1 \mathrm{~s}^{2} 2 \mathrm{~s}^{1} 3 \mathrm{p}^{1} 4 \mathrm{f}^{1}$, $1 s^{2} 3 s^{1} 3 p^{1} 4 p^{1}, 1 s^{2} 3 s^{1} 3 p^{1} 4 f^{1}, 1 s^{2} 2 p^{1} 3 p^{1} 4 s^{1}, 1 s^{2} 2 p^{1} 3 p^{1} 4 d^{1}$. The total number of CSFs in our MCDF calculations is 2253 .

\subsection{CITRO method}

In the nonrelativistic, multiconfiguration Hartree-Fock approach, intermediate coupling wavefunctions $\Psi_{\gamma}(J)$ being eigenfunctions of Coulomb-Breit-Pauli Hamiltonian are expanded in terms of CSFs $\Phi(\alpha L S J)$ obtained in the $L S J$-coupling scheme:

$\Psi_{\gamma}(J)=\sum_{\alpha L S} c_{\gamma}(\alpha L S J) \Phi(\alpha L S J)$

CSFs are formed from a basis of one-electron spin orbitals:

$\phi(r)=\frac{1}{r} P_{n l}(r) Y_{l m_{l}}(\vartheta, \phi) \chi_{m_{s}}$.

The number of CSFs in the intermediate coupling wavefunction expansion (5) is limited. Hence transformed radial orbitals with variable parameters were proposed by Bogdanovich \& Karpuškienè (1999) to mimic the correlation effects from omitted CSFs:

$P_{n l}^{\mathrm{T}}(r)=N\left\{f(r) P_{n l}(r)-\sum_{n^{\prime \prime}<n} c_{n^{\prime \prime}, n} P_{n^{\prime \prime} l}(r)\right\}$.

Here $N$ is a normalization factor, $c_{n^{\prime \prime}, n}$ denotes the corresponding overlap integral, and $f(r)$ is a transforming function:

$f(r)=r^{k} \exp \left(-A r^{m}\right)$

with variable parameters $k, m$ and $A\left(k \geq 0, k \geq l-l^{\prime}, m>\right.$ $0, A>0)$. The variation of all parameters ensure the largest corrections of correlation energies obtained in the second order of perturbation theory using admixed configurations with excited electrons. A Schmidt orthogonalization procedure is employed for TROs in (7).

The Coulomb-Breit-Pauli Hamiltonian is used for our configuration interaction calculations which lead to the expansion coefficients $c_{\gamma}(\alpha L S J)$ and the corresponding energies for levels. This Hamiltonian includes spin-orbit $h_{i}^{\text {so }}$, spin-other-orbit $h_{i j}^{\text {soo }}$ and spin-spin $h_{i j}^{\text {ss }}$ corrections as well as orbit-orbit corrections $h_{i j}^{\text {oo }}$ within a shell of equivalent electrons:

$$
\begin{aligned}
H^{\mathrm{BP}}= & \sum_{i}\left(-\frac{1}{2} \nabla_{i}^{2}-Z / r_{i}\right)+\sum_{i<j} \frac{1}{r_{i j}}+\sum_{i} h_{i}^{\mathrm{so}} \\
& +\sum_{i<j} h_{i j}^{\mathrm{soo}}+\sum_{i<j} h_{i j}^{\mathrm{ss}}+\sum_{i<j} h_{i j}^{\mathrm{oo}},
\end{aligned}
$$

where $H^{\mathrm{BP}}$ is expressed in atomic units. Orbit-orbit interactions between shells omitted in our CITRO calculations are usually smaller than within shells.

The frozen core approximation is used to obtain HartreeFock radial orbitals with $n \leq 7$. To include correlation effects from higher CSFs, the obtained basis is supplemented by TROs with principal quantum numbers $8 \leq n \leq 10$ and orbital 
quantum numbers $l \leq 7$. The large number of admixed configurations is reduced, leaving only configurations with significant influence on the energies of the adjusted configurations. For this, the method presented by Bogdanovich et al. (2002) and Bogdanovich \& Momkauskaitè (2004) is adopted. The total number of CSFs with odd parity is 2115 , and the number with even parity is 1987 in our CITRO calculations.

\section{Results and discussion}

In Table 1 we list energies of the 407 lowest levels of Fe XXII obtained with the GRASP code. These levels correspond to $1 \mathrm{~s}^{2} 2 \mathrm{~s}^{2} 2 \mathrm{p}^{1}, \quad 1 \mathrm{~s}^{2} 2 \mathrm{~s}^{1} 2 \mathrm{p}^{2}, \quad 1 \mathrm{~s}^{2} 2 \mathrm{p}^{3}, \quad 1 \mathrm{~s}^{2} 2 \mathrm{~s}^{2} 3 l, \quad 1 \mathrm{~s}^{2} 2 \mathrm{~s}^{1} 2 \mathrm{p}^{1} 3 l$, $1 \mathrm{~s}^{2} 2 \mathrm{p}^{2} 3 l, \quad 1 \mathrm{~s}^{2} 2 \mathrm{~s}^{2} 4 l^{\prime}, \quad 1 \mathrm{~s}^{2} 2 \mathrm{~s}^{1} 2 \mathrm{p}^{1} 4 l^{\prime}, \quad 1 \mathrm{~s}^{2} 2 \mathrm{p}^{2} 4 l^{\prime}, \quad 1 \mathrm{~s}^{2} 2 \mathrm{~s}^{2} 5 l^{\prime \prime}$, $1 \mathrm{~s}^{2} 2 \mathrm{~s}^{1} 2 \mathrm{p}^{1} 5 l^{\prime \prime}, 1 \mathrm{~s}^{2} 2 \mathrm{~s}^{2} 6 l^{\prime \prime \prime} \quad\left(l=0,1,2, l^{\prime}=0,1,2,3, l^{\prime \prime}=\right.$ $0,1,2,3,4$ and $\left.l^{\prime \prime \prime}=0,1,2,3,4,5\right)$ configurations. Energy levels are given in $\mathrm{cm}^{-1}$ relative to the ground state $1 \mathrm{~s}^{2} 2 \mathrm{~s}^{2} 2 \mathrm{p}^{1}{ }^{2} \mathrm{P}_{0.5}$, along with the leading percentage compositions $c_{\gamma}(\alpha J)^{2}$ (where these exceed 10\%) from the expansion relation Eq. (1) for intermediate coupling wavefunctions. The expansion coefficients for the intermediate coupling wavefunctions with CSFs presented in the $L S J$-coupling scheme (5) are obtained from the expansion terms of intermediate coupling wavefunctions with CSFs presented in $j j$-coupling scheme (1). Diagonalization of Dirac-Coulomb-Breit matrix gives the expansion coefficients for intermediate coupling wavefunctions with CSFs constructed from two-component orbitals. The indices for the levels provided in the first column of Table 1 are used in all subsequent tables.

Some excited levels due to strong intermediate coupling are assigned to the same CSFs. In Table 2 new $L S J$-coupling spectroscopic notations are proposed for the levels which have the same CSFs with primary contributions to the intermediate coupling wavefunctions. For this, the technique presented in our earlier paper for Fe XIX (Jonauskas et al. 2004b) is used.

In Table 3 we compare our calculated energy levels with values compiled by NIST (National Institute for Standards and Technology: www.physics.nist.gov) whose data are commonly used as a reference set. Fairly good agreement is obtained for the MCDF calculations, with the many energy levels agreeing to better than within $1 \%$ with the NIST recommended values. However, some excited energy levels (indices 29, 55, $59,69,74,174$ and 195) differ by up to $2 \%$ from NIST data. As well, our absolute values of energies for two levels (55 and 174) show the largest deviations from the NIST recommended values, while another five levels $(29,59,69,74$ and 195) have MCDF absolute energies lower than those from NIST. To cross-check our results for these levels, we present data from CITRO calculations. For both our calculations, the highly excited levels show the similar discrepancies from NIST values. On the other hand, the MCDF energies of levels $174\left(1 \mathrm{~s}^{2} 2 \mathrm{~s}^{1} 2 \mathrm{p}^{1}\right.$ $\left.\left({ }^{3} \mathrm{P}\right) 4 \mathrm{~d}^{1}{ }^{2} \mathrm{~F}_{3.5}\right)$ and $195\left(1 \mathrm{~s}^{2} 2 \mathrm{~s}^{1} 2 \mathrm{p}^{1}\left({ }^{1} \mathrm{P}\right) 4 \mathrm{~d}^{1}{ }^{2} \mathrm{~F}_{3.5}\right)$ show good agreement with NIST data after their values are interchanged. This suggests that there are some typographical errors in the NIST data for these levels. The calculated energies of level 29 $\left(1 \mathrm{~s}^{2} 2 \mathrm{~s}^{1} 2 \mathrm{p}^{1}\left({ }^{3} \mathrm{P}\right) 3 \mathrm{p}^{1}{ }^{2} \mathrm{P}_{0.5}\right)$ has similar discrepancies $(\sim 1.5 \%)$ for both calculations presented in Table 3 , but our MCDF result is closer to the NIST recommended value. As well, our CITRO and MCDF calculations show the same percentage composition for the intermediate coupling wavefunction of the level. The energy of the $1 s^{2} 2 s^{1} 2 p^{1}\left({ }^{3} \mathrm{P}\right) 3 \mathrm{p}^{1}{ }^{2} \mathrm{P}_{0.5}$ level (29) is lower than that of $1 \mathrm{~s}^{2} 2 \mathrm{~s}^{1} 2 \mathrm{p}^{1}\left({ }^{3} \mathrm{P}\right) 3 \mathrm{p}^{1}{ }^{2} \mathrm{P}_{1.5}$ (28) in the NIST data, while the ordering of both levels in our calculations is opposite to the NIST order. It suggests that a bigger CI basis may be required for the MCDF calculations to obtain better agreement with the NIST values for the level. However, the energy for the level obtained by Zhang \& Pradhan (1997) with SUPERSTRUCTURE (Eissner et al. 1974), and the value provided by Badnell et al. (2001) from their AUTOSTRUCTURE (Badnell 1986, 1997) calculations show similar differences from NIST, and the same order as in our data.

For another five levels $(55,59,69$ and 74) with the largest discrepancies from NIST values, our MCDF and CITRO energies are in close agreement with the AUTOSTRUCTURE values of Badnell et al. (2001) and, in general, are slightly closer to the NIST data. Furthermore, as will be seen later, some transitions from those levels have largest discrepancies for line strengths compared with the NIST values. Additionally, our calculated lifetimes for the levels are presented in Table 3, to have an additional way to estimate the accuracy of our calculations. Differences between the calculated lifetimes for those levels is less than $10 \%$, which confirm the accuracy of our results.

Energy levels and intermediate coupling wavefunctions calculated with the configuration interaction method have been employed to compute matrix elements of transition operators, which subsequently are adopted for the calculation of transition probabilities, line and oscillator strengths. The wavelengths and line strengths are presented in Table 4, where our transition data are compared with NIST results. All line strengths belong to E1 type transitions except one from the first excited level which corresponds to M1 type transition. E2 type transition from the level is much weaker (MCDF and NIST line strengths equal to $2.10 \times 10^{-3}$ and $2.15 \times 10^{-3}$ correspondingly). The length form is used for electric transitions, as they are less sensitive to the accuracy of wavefunction compared with results obtained in the velocity form of the transition operator. The wavelengths for many transitions agree to better than within $1 \%$ with the NIST data. The largest differences for the wavelengths are obtained for transitions between energy levels which showed largest discrepancies from NIST energies. The largest discrepancy for line strengths is observed for the weak transition $\left(f=3 \times 10^{-5}\right)$ from the $2 \mathrm{~s}^{1} 2 \mathrm{p}^{2}{ }^{2} \mathrm{P}_{0.5}$ level (8) to $2 \mathrm{~s}^{2} 2 \mathrm{p}^{12} \mathrm{P}_{1.5}$ (2). The length and velocity forms differ by a factor of 3.5, while our calculated MCDF and CITRO line strengths coincide within $20 \%$ for the transition. Furthermore, the contribution of the transition to the lifetime of the level is very small. The line strengths for the transitions from $2 \mathrm{~s}^{2} 4 \mathrm{~s}^{1}{ }^{2} \mathrm{~S}_{0.5}$ (126) to the first and second levels of the ground configuration differ by $80 \%$ and $29 \%$, respectively, from the NIST values, but are in the limits of uncertainties provided by NIST.

Additionally, large discrepancies are observed for some transitions from the above mentioned levels which showed the largest deviations for energy levels compared with the NIST values. The line strengths for transitions $55 \rightarrow 9,55 \rightarrow 10,59 \rightarrow 8$, $69 \rightarrow 8,74 \rightarrow 8,174 \rightarrow 7$ and $195 \rightarrow 7$ differ by $29 \%$ to up to a factor of 4 . The contributions to the lifetimes of those dipole allowed transitions vary from $7 \%(74 \rightarrow 8)$ to $84 \%(195 \rightarrow 7)$ (Table 5). The large discrepancies observed for transitions from levels 174 and 195 can be explained by a typographical error in the NIST data. As in a case of energy levels, our line strengths and wavelengths for those transitions are in good agreement with the NIST line strengths, after the NIST values are interchanged. Finally, our MCDF results for all those transitions with large discrepancies with NIST line strengths are in close agreement with the CITRO ones. It is possible that the determination of all those energy levels is uncertain in the NIST database. Additionally, the fairly good agreement between length and velocity forms for all 
those transitions indicate that our transition characteristics are quite accurate and reliable.

Transition probabilities, line and oscillator strengths are calculated for radiative E1, E2, E3, M1 and M2 type transitions among the presented 407 energy levels of Fe XXII. We provide the transition wavelengths, probabilities, oscillator strengths and line strengths of E1, E2 and M1 type for Fe XXII in Tables 6-8. Only the strongest transitions are presented here for every type of transition: electric dipole transitions with $f \geq 0.001$, electric quadrupole with $f \geq 10^{-8}$ and magnetic dipole with $f \geq 10^{-8}$. The relation between length (Babushkin gauge in the relativistic approach) and velocity (Coulomb gauge) forms are provided for both electric transitions to have an additional indicator of accuracy of our MCDF results. The difference between both forms for many strong E1 type transitions $(f \geq 0.1)$ does not exceed $20 \%$. Only 4 transitions from highly excited levels have differences larger than $20 \%$ but less than $40 \%$. On the other hand, the contribution of these transitions to the lifetimes of the levels is much smaller than $1 \%$. Finally, the length and velocity forms of the strongest electric dipole transitions $(f \geq 0.1)$ agree to better than within $10 \%$, with an average deviation of only $1.8 \%$ for 457 transitions. For many E2 transitions, the two forms agree to better than within $10 \%$.

In Table 5 we provide the five largest spontaneous radiative transition probabilities from each level, and the sum of all E1, E2, E3, M1 and M2 radiative transition probabilities from the corresponding level to all lower levels. The sums of radiative transition probabilities are important for branching ratios, while their inverse values are equal to the lifetimes of the levels. The contribution to the lifetimes of levels for many forbidden electric quadrupole and magnetic dipole transitions is negligible and does not exceed $1 \%$. However, the highly excited $2 s^{1} 2 p^{1}\left({ }^{3} \mathrm{P}\right)$ $3 \mathrm{p}^{1}{ }^{4} \mathrm{D}_{0.5}$ (33) and $2 \mathrm{p}^{2}\left({ }^{1} \mathrm{D}\right) 3 \mathrm{~d}^{1}{ }^{2} \mathrm{G}_{0.5}$ (114) levels decay primarily through $\mathrm{E} 2$ type transitions. For another two levels, i.e. $2 \mathrm{p}^{2}\left({ }^{3} \mathrm{P}\right) 3 \mathrm{~d}^{1}{ }^{4} \mathrm{D}_{0.5}(97)$ and $2 \mathrm{p}^{2}\left({ }^{3} \mathrm{P}\right) 3 \mathrm{~d}^{1}{ }^{4} \mathrm{~F}_{0.5}(96)$, E2 type transitions contribute $48 \%$ and $46 \%$, respectively. As well, the first excited level decays mainly due to magnetic dipole transition.

\section{Conclusions}

In this paper, energy levels, electric dipole, electric quadrupole and magnetic dipole radiative transition rates, oscillator and line strengths have been reported for the lowest 407 levels in Fe XXII in the MCDF approximation. The characteristics of the levels in the $L S J$-coupling scheme have been provided and checked for their completeness. Calculated values have been compared with NIST recommended values. Breit-Pauli energy levels and electric dipole transition characteristics in the basis set of transformed radial orbitals with variable parameters were used to cross-check the accuracy of our results. Likely typographical errors for Fe XXII have been found in both the NIST energy levels and transition characteristics for the highly excited $2 \mathrm{~s}^{1} 2 \mathrm{p}^{1}\left({ }^{3} \mathrm{P}\right) 4 \mathrm{~d}^{1}{ }^{2} \mathrm{~F}_{3.5}$ and $2 \mathrm{~s}^{1} 2 \mathrm{p}^{1}\left({ }^{1} \mathrm{P}\right) 4 \mathrm{~d}^{1}{ }^{2} \mathrm{~F}_{3.5}$ levels.
The forbidden transitions have been taken into account to obtain total radiative transition probabilities from levels. The total radiative probabilities allow estimates of the lifetimes of the presented states, and are also important for the calculation of branching ratios. It was found that the highly excited $2 \mathrm{~s}^{1} 2 \mathrm{p}^{1}\left({ }^{3} \mathrm{P}\right) 3 \mathrm{p}^{1}{ }^{4} \mathrm{D}_{0.5}$ and $2 \mathrm{p}^{2}\left({ }^{1} \mathrm{D}\right) 3 \mathrm{~d}^{12} \mathrm{G}_{0.5}$ levels mainly decay due to forbidden $\mathrm{E} 2$ type transitions. Their contributions to the lifetimes of the $2 \mathrm{p}^{2}\left({ }^{3} \mathrm{P}\right) 3 \mathrm{~d}^{1{ }^{4}} \mathrm{D}_{0.5}$ and $2 \mathrm{p}^{2}\left({ }^{3} \mathrm{P}\right) 3 \mathrm{~d}^{1}{ }^{4} \mathrm{~F}_{0.5}$ levels is $48 \%$ and $46 \%$, respectively. The first excited level decays mainly through M1 type transition. For each level, the five strongest transition probabilities are presented.

To conclude, the comparison with the NIST recommended wavelengths and radiative transition data shows that all the presented theoretical results are reliable, and may be successfully used for the interpretation of astronomical and other spectral observations.

Acknowledgements. FPK and SJR are grateful to AWE Aldermaston for the award of William Penney Fellowships. This work was supported by PPARC and EPSRC, and also by NATO Collaborative Linkage Grant CLG.979443. We are also grateful to the Defence Science and Technology Laboratory (dstl) for support under the Joint Grants Scheme.

\section{References}

Badnell, N. R. 1986, J. Phys. B, 19, 3827

Badnell, N. R. 1997, J. Phys. B, 30, 1

Badnell, N. R., Griffin, D. C., \& Mitnik, D. M. 2001, J. Phys. B, 34, 5071

Bar-Shalom, A., Klapisch, M., \& Oreg, J. 2001, J. Quant. Spectr. Radiat. Trans., 71,169

Behar, E., Cottam, J., \& Kahn, S. M. 2001, ApJ, 548, 966

Bogdanovich, P., \& Karpuškienė, R. 1999, Lithuan. J. Phys., 39, 193

Bogdanovich, P., Karpuškienè, R., \& Momkauskaitė, A. 2002, Comput. Phys. Comm., 143, 174

Bogdanovich, P., \& Momkauskaite, A. 2004, Comput. Phys. Comm., 157, 217

Chen, H., Beiersdorfer, P., Heeter, L. A., et al. 2004, ApJ, 611, 598

Doschek, G. A., Feldman, U., \& Cowan, R. D. 1981, ApJ, 245, 315

Doschek, G. A., Meekins, J. F., \& Cowan, R. D. 1973, Sol. Phys., 29, 125

Eissner, W., Jones, M., \& Nussbaumer, H. 1974, Comput. Phys. Commun., 8, 270

Fawcett, B. C., Phillips, K. J. H., Jordan, C., \& Lemen, J. R. 1987, MNRAS, 225,1013

Grant, I. P., McKenzie, B. J., Norrington, P. H., Mayers, D. F., \& Pyper, N. C. 1980, Comput. Phys. Comm., 21, 207

Jonauskas, V., Keenan, F. P., Foord, M. E., et al. 2004b, A\&A, 416, 383

Jonauskas, V., Keenan, F. P., Foord, M. E., et al. 2004a, A\&A, 424, 363

Jonauskas, V., Bogdanovich, P., Keenan, F. P., et al. 2005, A\&A, 433, 745

Kinkhabwala, A., Sako, M., Behar, E., et al. 2002, ApJ, 575, 732

McKenzie, D. L., Landecker, P. B., Feldman, U., \& Doschek, G. A. 1985, ApJ, 289, 849

Parpia, F. A., Froese Fischer, C., \& Grant, I. P. 1996, Comput. Phys. Comm., 94, 249

Phillips, K. J. H., Bhatia, A. K., Mason, H. E., \& Zarro, D. M. 1996, ApJ, 466, 549

Phillips, K. J. H., Fawcett, B. C., Kent, B. J., et al. 1982, ApJ, 256, 774

Savin, D. 2001, in Spectroscopic Challenges of Photoionized Plasmas, ASP Conf. Ser., 247, 167

Wargelin, B. J., Beiersdorfer, P., Liedahl, D. A., Kahn, S. M., \& von Goeler, S. 1998, ApJ, 496, 1031

Zhang, H. L., \& Pradhan, A. K. 1997, A\&AS, 123, 575 


\section{Online Material}


V. Jonauskas et al.: Transition rates for Fe XXII, Online Material $p 2$

Table 1. MCDF results for energy levels of Fe XXII. The energies of levels are presented relative to the ground energy. The leading percentage compositions $(>10 \%)$ of levels are provided in the last column.

\begin{tabular}{|c|c|c|c|c|c|}
\hline Index & Configuration & $L S$ & $J$ & $E\left(\mathrm{~cm}^{-1}\right)$ & Composition \\
\hline 1 & $1 s^{2} 2 s^{2} 2 p^{1}$ & ${ }^{2} \mathrm{P}$ & 0.5 & -192757192 & $98 \%$ \\
\hline 2 & $1 s^{2} 2 s^{2} 2 p^{1}$ & ${ }^{2} \mathrm{P}$ & 1.5 & 117953 & $97 \%$ \\
\hline 3 & $1 s^{2} 2 s^{1} 2 p^{2}$ & ${ }^{4} \mathrm{P}$ & 0.5 & 402902 & $96 \%$ \\
\hline 4 & $1 s^{2} 2 s^{1} 2 p^{2}$ & ${ }^{4} \mathrm{P}$ & 1.5 & 458031 & $99 \%$ \\
\hline 5 & $1 s^{2} 2 s^{1} 2 p^{2}$ & ${ }^{4} \mathrm{P}$ & 2.5 & 511295 & $94 \%$ \\
\hline 6 & $1 s^{2} 2 s^{1} 2 p^{2}$ & ${ }^{2} \mathrm{D}$ & 1.5 & 741538 & $95 \%$ \\
\hline 7 & $1 s^{2} 2 s^{1} 2 p^{2}$ & ${ }^{2} \mathrm{D}$ & 2.5 & 763743 & $94 \%$ \\
\hline 8 & $1 s^{2} 2 s^{1} 2 p^{2}$ & ${ }^{2} \mathrm{P}$ & 0.5 & 861921 & $60 \%+37 \% 1 \mathrm{~s}^{2} 2 \mathrm{~s}^{1} 2 \mathrm{p}^{2}{ }^{2} \mathrm{~S}$ \\
\hline 9 & $1 s^{2} 2 s^{1} 2 p^{2}$ & ${ }^{2} \mathrm{~S}$ & 0.5 & 986277 & $59 \%+39 \% 1 \mathrm{~s}^{2} 2 \mathrm{~s}^{1} 2 \mathrm{p}^{2}{ }^{2} \mathrm{P}$ \\
\hline 10 & $1 s^{2} 2 s^{1} 2 p^{2}$ & ${ }^{2} \mathrm{P}$ & 1.5 & 1000692 & $95 \%$ \\
\hline 11 & $1 s^{2} 2 p^{3}$ & ${ }^{4} S$ & 1.5 & 1260179 & $90 \%$ \\
\hline 12 & $1 \mathrm{~s}^{2} 2 \mathrm{p}^{3}$ & ${ }^{2} \mathrm{D}$ & 1.5 & 1405688 & $82 \%+12 \% 1 \mathrm{~s}^{2} 2 \mathrm{p}^{32} \mathrm{P}$ \\
\hline 13 & $1 \mathrm{~s}^{2} 2 \mathrm{p}^{3}$ & ${ }^{2} \mathrm{D}$ & 2.5 & 1435674 & $100 \%$ \\
\hline 14 & $1 \mathrm{~s}^{2} 2 \mathrm{p}^{3}$ & ${ }^{2} \mathrm{P}$ & 0.5 & 1582452 & $98 \%$ \\
\hline 15 & $1 \mathrm{~s}^{2} 2 \mathrm{p}^{3}$ & ${ }^{2} \mathrm{P}$ & 1.5 & 1639226 & $78 \%+16 \% 1 \mathrm{~s}^{2} 2 \mathrm{p}^{3}{ }^{2} \mathrm{D}$ \\
\hline 16 & $1 s^{2} 2 s^{2} 3 s^{1}$ & ${ }^{2} \mathrm{~S}$ & 0.5 & 8111550 & $95 \%$ \\
\hline 17 & $1 s^{2} 2 s^{2} 3 p^{1}$ & ${ }^{2} \mathrm{P}$ & 0.5 & 8292834 & $91 \%$ \\
\hline 18 & $1 s^{2} 2 s^{2} 3 p^{1}$ & ${ }^{2} \mathrm{P}$ & 1.5 & 8326008 & $91 \%$ \\
\hline 19 & $1 s^{2} 2 s^{1} 2 p^{1}\left({ }^{3} P\right) 3 s^{1}$ & ${ }^{4} \mathrm{P}$ & 0.5 & 8471848 & $96 \%$ \\
\hline 20 & $1 \mathrm{~s}^{2} 2 \mathrm{~s}^{2} 3 \mathrm{~d}^{1}$ & ${ }^{2} \mathrm{D}$ & 1.5 & 8491152 & $95 \%$ \\
\hline 21 & $1 s^{2} 2 s^{2} 3 d^{1}$ & ${ }^{2} \mathrm{D}$ & 2.5 & 8501013 & $95 \%$ \\
\hline 22 & $1 s^{2} 2 s^{1} 2 p^{1}\left({ }^{3} P\right) 3 s^{1}$ & ${ }^{4} \mathrm{P}$ & 1.5 & 8502156 & $91 \%$ \\
\hline 23 & $1 s^{2} 2 s^{1} 2 p^{1}\left({ }^{3} P\right) 3 s^{1}$ & ${ }^{2} \mathrm{P}$ & 0.5 & 8577184 & $92 \%$ \\
\hline 24 & $1 s^{2} 2 s^{1} 2 p^{1}\left({ }^{3} P\right) 3 s^{1}$ & ${ }^{4} \mathrm{P}$ & 2.5 & 8584529 & $98 \%$ \\
\hline 25 & $1 s^{2} 2 s^{1} 2 p^{1}\left({ }^{3} P\right) 3 p^{1}$ & ${ }^{4} \mathrm{D}$ & 0.5 & 8630313 & $76 \%+21 \% 1 \mathrm{~s}^{2} 2 \mathrm{~s}^{1} 2 \mathrm{p}^{1}\left({ }^{3} \mathrm{P}\right) 3 \mathrm{p}^{12} \mathrm{P}$ \\
\hline 26 & $1 s^{2} 2 s^{1} 2 p^{1}\left({ }^{3} P\right) 3 p^{1}$ & ${ }^{4} \mathrm{D}$ & 1.5 & 8657024 & $75 \%+18 \% 1 \mathrm{~s}^{2} 2 \mathrm{~s}^{1} 2 \mathrm{p}^{1}\left({ }^{3} \mathrm{P}\right) 3 \mathrm{p}^{12} \mathrm{P}$ \\
\hline 27 & $1 s^{2} 2 s^{1} 2 p^{1}\left({ }^{3} P\right) 3 s^{1}$ & ${ }^{2} \mathrm{P}$ & 1.5 & 8663895 & $90 \%$ \\
\hline 28 & $1 s^{2} 2 s^{1} 2 p^{1}\left({ }^{3} P\right) 3 p^{1}$ & ${ }^{2} \mathrm{P}$ & 1.5 & 8695560 & $30 \%+25 \% 1 s^{2} 2 s^{1} 2 p^{1}\left({ }^{3} P\right) 3 p^{14} S+17 \% 1 s^{2} 2 s^{1} 2 p^{1}\left({ }^{3} P\right) 3 p^{14} P$ \\
\hline 29 & $1 s^{2} 2 s^{1} 2 p^{1}\left({ }^{3} P\right) 3 p^{1}$ & ${ }^{2} \mathrm{P}$ & 0.5 & 8704356 & $66 \%+22 \% 1 s^{2} 2 s^{1} 2 p^{1}\left({ }^{3} P\right) 3 p^{14} D$ \\
\hline 30 & $1 s^{2} 2 s^{1} 2 p^{1}\left({ }^{3} P\right) 3 p^{1}$ & ${ }^{4} \mathrm{D}$ & 2.5 & 8707420 & $90 \%$ \\
\hline 31 & $1 s^{2} 2 s^{1} 2 p^{1}\left({ }^{3} P\right) 3 p^{1}$ & ${ }^{4} \mathrm{P}$ & 0.5 & 8734123 & $90 \%$ \\
\hline 32 & $1 s^{2} 2 s^{1} 2 p^{1}\left({ }^{3} P\right) 3 p^{1}$ & ${ }^{2} \mathrm{D}$ & 1.5 & 8748534 & $36 \%+31 \% 1 s^{2} 2 s^{1} 2 p^{1}\left({ }^{3} P\right) 3 p^{14} S+15 \% 1 s^{2} 2 s^{1} 2 p^{1}\left({ }^{3} P\right) 3 p^{1}{ }^{4} P$ \\
\hline 33 & $1 s^{2} 2 s^{1} 2 p^{1}\left({ }^{3} P\right) 3 p^{1}$ & ${ }^{4} \mathrm{D}$ & 3.5 & 8781923 & $99 \%$ \\
\hline 34 & $1 s^{2} 2 s^{1} 2 p^{1}\left({ }^{3} P\right) 3 p^{1}$ & ${ }^{4} \mathrm{P}$ & 1.5 & 8791861 & $44 \%+25 \% 1 \mathrm{~s}^{2} 2 \mathrm{~s}^{1} 2 \mathrm{p}^{1}\left({ }^{3} \mathrm{P}\right) 3 \mathrm{p}^{12} \mathrm{D}+19 \% 1 \mathrm{~s}^{2} 2 \mathrm{~s}^{1} 2 \mathrm{p}^{1}\left({ }^{3} \mathrm{P}\right) 3 \mathrm{p}^{12} \mathrm{P}$ \\
\hline 35 & $1 s^{2} 2 s^{1} 2 p^{1}\left({ }^{3} P\right) 3 d^{1}$ & ${ }^{4} \mathrm{~F}$ & 1.5 & 8808079 & $89 \%$ \\
\hline 36 & $1 s^{2} 2 s^{1} 2 p^{1}\left({ }^{3} P\right) 3 p^{1}$ & ${ }^{4} S$ & 1.5 & 8809920 & $37 \%+22 \% 1 \mathrm{~s}^{2} 2 \mathrm{~s}^{1} 2 \mathrm{p}^{1}\left({ }^{3} \mathrm{P}\right) 3 \mathrm{p}^{14} \mathrm{P}+19 \% 1 \mathrm{~s}^{2} 2 \mathrm{~s}^{1} 2 \mathrm{p}^{1}\left({ }^{3} \mathrm{P}\right) 3 \mathrm{p}^{12} \mathrm{D}$ \\
\hline 37 & $1 s^{2} 2 s^{1} 2 p^{1}\left({ }^{3} P\right) 3 p^{1}$ & ${ }^{4} \mathrm{P}$ & 2.5 & 8809932 & $68 \%+23 \% 1 \mathrm{~s}^{2} 2 \mathrm{~s}^{1} 2 \mathrm{p}^{1}\left({ }^{3} \mathrm{P}\right) 3 \mathrm{p}^{12} \mathrm{D}$ \\
\hline 38 & $1 s^{2} 2 s^{1} 2 p^{1}\left({ }^{3} P\right) 3 d^{1}$ & ${ }^{4} \mathrm{~F}$ & 2.5 & 8827013 & $85 \%+11 \% 1 \mathrm{~s}^{2} 2 \mathrm{~s}^{1} 2 \mathrm{p}^{1}\left({ }^{3} \mathrm{P}\right) 3 \mathrm{~d}^{14} \mathrm{D}$ \\
\hline 39 & $1 s^{2} 2 s^{1} 2 p^{1}\left({ }^{3} P\right) 3 p^{1}$ & ${ }^{2} \mathrm{D}$ & 2.5 & 8857624 & $73 \%+24 \% 1 \mathrm{~s}^{2} 2 \mathrm{~s}^{1} 2 \mathrm{p}^{1}\left({ }^{3} \mathrm{P}\right) 3 \mathrm{p}^{14} \mathrm{P}$ \\
\hline 40 & $1 s^{2} 2 s^{1} 2 p^{1}\left({ }^{3} P\right) 3 d^{1}$ & ${ }^{4} \mathrm{~F}$ & 3.5 & 8857700 & $79 \%+17 \% 1 \mathrm{~s}^{2} 2 \mathrm{~s}^{1} 2 \mathrm{p}^{1}\left({ }^{3} \mathrm{P}\right) 3 \mathrm{~d}^{14} \mathrm{D}$ \\
\hline 41 & $1 s^{2} 2 s^{1} 2 p^{1}\left({ }^{3} P\right) 3 d^{1}$ & ${ }^{4} \mathrm{P}$ & 2.5 & 8868503 & $46 \%+23 \% 1 \mathrm{~s}^{2} 2 \mathrm{~s}^{1} 2 \mathrm{p}^{1}\left({ }^{3} \mathrm{P}\right) 3 \mathrm{~d}^{14} \mathrm{D}+23 \% 1 \mathrm{~s}^{2} 2 \mathrm{~s}^{1} 2 \mathrm{p}^{1}\left({ }^{3} \mathrm{P}\right) 3 \mathrm{~d}^{12} \mathrm{D}$ \\
\hline 42 & $1 s^{2} 2 s^{1} 2 p^{1}\left({ }^{3} P\right) 3 d^{1}$ & ${ }^{4} \mathrm{D}$ & 1.5 & 8878309 & $59 \%+25 \% 1 \mathrm{~s}^{2} 2 \mathrm{~s}^{1} 2 \mathrm{p}^{1}\left({ }^{3} \mathrm{P}\right) 3 \mathrm{~d}^{14} \mathrm{P}$ \\
\hline 43 & $1 s^{2} 2 s^{1} 2 p^{1}\left({ }^{3} P\right) 3 d^{1}$ & ${ }^{4} \mathrm{D}$ & 0.5 & 8881447 & $84 \%$ \\
\hline 44 & $1 s^{2} 2 s^{1} 2 p^{1}\left({ }^{3} P\right) 3 p^{1}$ & ${ }^{2} S$ & 0.5 & 8888579 & $79 \%$ \\
\hline 45 & $1 s^{2} 2 s^{1} 2 p^{1}\left({ }^{1} \mathrm{P}\right) 3 s^{1}$ & ${ }^{2} \mathrm{P}$ & 0.5 & 8891445 & $91 \%$ \\
\hline 46 & $1 s^{2} 2 s^{1} 2 p^{1}\left({ }^{1} \mathrm{P}\right) 3 s^{1}$ & ${ }^{2} \mathrm{P}$ & 1.5 & 8892983 & $82 \%$ \\
\hline 47 & $1 s^{2} 2 s^{1} 2 p^{1}\left({ }^{3} P\right) 3 d^{1}$ & ${ }^{2} \mathrm{D}$ & 1.5 & 8916505 & $67 \%+11 \% 1 \mathrm{~s}^{2} 2 \mathrm{~s}^{1} 2 \mathrm{p}^{1}\left({ }^{3} \mathrm{P}\right) 3 \mathrm{~d}^{12} \mathrm{P}$ \\
\hline 48 & $1 s^{2} 2 s^{1} 2 p^{1}\left({ }^{3} P\right) 3 d^{1}$ & ${ }^{4} \mathrm{~F}$ & 4.5 & 8925683 & $100 \%$ \\
\hline 49 & $1 s^{2} 2 s^{1} 2 p^{1}\left({ }^{3} P\right) 3 d^{1}$ & ${ }^{2} \mathrm{D}$ & 2.5 & 8930222 & $43 \%+28 \% 1 s^{2} 2 s^{1} 2 p^{1}\left({ }^{3} \mathrm{P}\right) 3 d^{12} \mathrm{~F}+13 \% 1 s^{2} 2 s^{1} 2 p^{1}\left({ }^{3} \mathrm{P}\right) 3 \mathrm{~d}^{14} \mathrm{D}$ \\
\hline 50 & $1 s^{2} 2 s^{1} 2 p^{1}\left({ }^{3} P\right) 3 d^{1}$ & ${ }^{4} \mathrm{D}$ & 3.5 & 8955370 & $79 \%+19 \% 1 \mathrm{~s}^{2} 2 \mathrm{~s}^{1} 2 \mathrm{p}^{1}\left({ }^{3} \mathrm{P}\right) 3 \mathrm{~d}^{14} \mathrm{~F}$ \\
\hline 51 & $1 s^{2} 2 s^{1} 2 p^{1}\left({ }^{3} P\right) 3 d^{1}$ & ${ }^{4} \mathrm{D}$ & 2.5 & 8966348 & $50 \%+35 \% 1 \mathrm{~s}^{2} 2 \mathrm{~s}^{1} 2 \mathrm{p}^{1}\left({ }^{3} \mathrm{P}\right) 3 \mathrm{~d}^{14} \mathrm{P}$ \\
\hline 52 & $1 s^{2} 2 s^{1} 2 p^{1}\left({ }^{3} P\right) 3 d^{1}$ & ${ }^{4} \mathrm{P}$ & 1.5 & 8972247 & $68 \%+29 \% 1 \mathrm{~s}^{2} 2 \mathrm{~s}^{1} 2 \mathrm{p}^{1}\left({ }^{3} \mathrm{P}\right) 3 \mathrm{~d}^{14} \mathrm{D}$ \\
\hline 53 & $1 s^{2} 2 s^{1} 2 p^{1}\left({ }^{3} P\right) 3 d^{1}$ & ${ }^{4} \mathrm{P}$ & 0.5 & 8975643 & $91 \%$ \\
\hline 54 & $1 s^{2} 2 s^{1} 2 p^{1}\left({ }^{3} P\right) 3 d^{1}$ & ${ }^{2} \mathrm{~F}$ & 2.5 & 9004790 & $55 \%+28 \% 1 \mathrm{~s}^{2} 2 \mathrm{~s}^{1} 2 \mathrm{p}^{1}\left({ }^{3} \mathrm{P}\right) 3 \mathrm{~d}^{12} \mathrm{D}$ \\
\hline 55 & $1 s^{2} 2 s^{1} 2 p^{1}\left({ }^{3} P\right) 3 d^{1}$ & ${ }^{2} \mathrm{P}$ & 1.5 & 9043521 & $78 \%+10 \% 1 \mathrm{~s}^{2} 2 \mathrm{~s}^{1} 2 \mathrm{p}^{1}\left({ }^{3} \mathrm{P}\right) 3 \mathrm{~d}^{12} \mathrm{D}$ \\
\hline 56 & $1 s^{2} 2 s^{1} 2 p^{1}\left({ }^{3} P\right) 3 d^{1}$ & ${ }^{2} \mathrm{~F}$ & 3.5 & 9060831 & $97 \%$ \\
\hline 57 & $1 s^{2} 2 s^{1} 2 p^{1}\left({ }^{1} P\right) 3 p^{1}$ & ${ }^{2} \mathrm{P}$ & 0.5 & 9067791 & $79 \%+10 \% 1 \mathrm{~s}^{2} 2 \mathrm{p}^{2}\left({ }^{3} \mathrm{P}\right) 3 \mathrm{~s}^{1}{ }^{2} \mathrm{P}$ \\
\hline 58 & $1 s^{2} 2 s^{1} 2 p^{1}\left({ }^{1} P\right) 3 p^{1}$ & ${ }^{2} \mathrm{D}$ & 1.5 & 9074412 & $64 \%+26 \% 1 \mathrm{~s}^{2} 2 \mathrm{~s}^{1} 2 \mathrm{p}^{1}\left({ }^{1} \mathrm{P}\right) 3 \mathrm{p}^{1}{ }^{2} \mathrm{P}$ \\
\hline 59 & $1 s^{2} 2 s^{1} 2 p^{1}\left({ }^{3} P\right) 3 d^{1}$ & ${ }^{2} \mathrm{P}$ & 0.5 & 9078984 & $94 \%$ \\
\hline 60 & $1 \mathrm{~s}^{2} 2 \mathrm{~s}^{1} 2 \mathrm{p}^{1}\left({ }^{1} \mathrm{P}\right) 3 \mathrm{p}^{1}$ & ${ }^{2} \mathrm{D}$ & 2.5 & 9093833 & $91 \%$ \\
\hline
\end{tabular}


Table 1. continued.

\begin{tabular}{|c|c|c|c|c|c|}
\hline Index & Configuration & $L S$ & $J$ & $E\left(\mathrm{~cm}^{-1}\right)$ & Composition \\
\hline 61 & $1 s^{2} 2 s^{1} 2 p^{1}\left({ }^{1} P\right) 3 p^{1}$ & ${ }^{2} \mathrm{P}$ & 1.5 & 9103377 & $59 \%+25 \% 1 \mathrm{~s}^{2} 2 \mathrm{~s}^{1} 2 \mathrm{p}^{1}\left({ }^{1} \mathrm{P}\right) 3 \mathrm{p}^{12} \mathrm{D}$ \\
\hline 62 & $1 s^{2} 2 p^{2}\left({ }^{3} P\right) 3 s^{1}$ & ${ }^{4} \mathrm{P}$ & 0.5 & 9121949 & $55 \%+27 \% 1 \mathrm{~s}^{2} 2 \mathrm{~s}^{1} 2 \mathrm{p}^{1}\left({ }^{1} \mathrm{P}\right) 3 \mathrm{p}^{12} \mathrm{~s}$ \\
\hline 63 & $1 s^{2} 2 s^{1} 2 p^{1}\left({ }^{1} P\right) 3 p^{1}$ & ${ }^{2} \mathrm{~S}$ & 0.5 & 9153543 & $54 \%+33 \% 1 \mathrm{~s}^{2} 2 \mathrm{p}^{2}\left({ }^{3} \mathrm{P}\right) 3 \mathrm{~s}^{1}{ }^{4} \mathrm{P}$ \\
\hline 64 & $1 \mathrm{~s}^{2} 2 \mathrm{p}^{2}\left({ }^{3} \mathrm{P}\right) 3 \mathrm{~s}^{1}$ & ${ }^{4} \mathrm{P}$ & 1.5 & 9193266 & $95 \%$ \\
\hline 65 & $1 s^{2} 2 p^{2}\left({ }^{3} P\right) 3 s^{1}$ & ${ }^{4} \mathrm{P}$ & 2.5 & 9238570 & $80 \%+16 \% 1 \mathrm{~s}^{2} 2 \mathrm{p}^{2}\left({ }^{1} \mathrm{D}\right) 3 \mathrm{~s}^{12} \mathrm{D}$ \\
\hline 66 & $1 s^{2} 2 s^{1} 2 p^{1}\left({ }^{1} \mathrm{P}\right) 3 d^{1}$ & ${ }^{2} \mathrm{~F}$ & 3.5 & 9250872 & $93 \%$ \\
\hline 67 & $1 \mathrm{~s}^{2} 2 \mathrm{~s}^{1} 2 \mathrm{p}^{1}\left({ }^{1} \mathrm{P}\right) 3 \mathrm{~d}^{1}$ & ${ }^{2} \mathrm{~F}$ & 2.5 & 9253139 & $85 \%$ \\
\hline 68 & $1 \mathrm{~s}^{2} 2 \mathrm{p}^{2}\left({ }^{3} \mathrm{P}\right) 3 \mathrm{~s}^{1}$ & ${ }^{2} \mathrm{P}$ & 0.5 & 9262279 & $77 \%$ \\
\hline 69 & $1 \mathrm{~s}^{2} 2 \mathrm{~s}^{1} 2 \mathrm{p}^{1}\left({ }^{1} \mathrm{P}\right) 3 \mathrm{~d}^{1}$ & ${ }^{2} \mathrm{D}$ & 1.5 & 9263777 & $93 \%$ \\
\hline 70 & $1 s^{2} 2 p^{2}\left({ }^{3} \mathrm{P}\right) 3 p^{1}$ & ${ }^{4} \mathrm{D}$ & 0.5 & 9265730 & $50 \%+16 \% 1 \mathrm{~s}^{2} 2 \mathrm{p}^{2}\left({ }^{3} \mathrm{P}\right) 3 \mathrm{p}^{1}{ }^{2} \mathrm{~S}$ \\
\hline 71 & $1 \mathrm{~s}^{2} 2 \mathrm{~s}^{1} 2 \mathrm{p}^{1}\left({ }^{1} \mathrm{P}\right) 3 \mathrm{~d}^{1}$ & ${ }^{2} \mathrm{D}$ & 2.5 & 9276912 & $90 \%$ \\
\hline 72 & $1 s^{2} 2 p^{2}\left({ }^{3} P\right) 3 s^{1}$ & ${ }^{2} \mathrm{P}$ & 1.5 & 9298666 & $47 \%+41 \% 1 \mathrm{~s}^{2} 2 \mathrm{p}^{2}\left({ }^{1} \mathrm{D}\right) 3 \mathrm{~s}^{12} \mathrm{D}$ \\
\hline 73 & $1 s^{2} 2 s^{1} 2 p^{1}\left({ }^{1} P\right) 3 d^{1}$ & ${ }^{2} \mathrm{P}$ & 0.5 & 9298930 & $89 \%$ \\
\hline 74 & $1 \mathrm{~s}^{2} 2 \mathrm{~s}^{1} 2 \mathrm{p}^{1}\left({ }^{1} \mathrm{P}\right) 3 \mathrm{~d}^{1}$ & ${ }^{2} \mathrm{P}$ & 1.5 & 9300838 & $62 \%+18 \% 1 \mathrm{~s}^{2} 2 \mathrm{p}^{2}\left({ }^{3} \mathrm{P}\right) 3 \mathrm{p}^{14} \mathrm{D}$ \\
\hline 75 & $1 s^{2} 2 p^{2}\left({ }^{3} P\right) 3 p^{1}$ & ${ }^{4} \mathrm{D}$ & 1.5 & 9318728 & $53 \%+32 \% 1 \mathrm{~s}^{2} 2 \mathrm{~s}^{1} 2 \mathrm{p}^{1}\left({ }^{1} \mathrm{P}\right) 3 \mathrm{~d}^{1}{ }^{2} \mathrm{P}$ \\
\hline 76 & $1 \mathrm{~s}^{2} 2 \mathrm{p}^{2}\left({ }^{3} \mathrm{P}\right) 3 \mathrm{p}^{1}$ & ${ }^{2} \mathrm{~S}$ & 0.5 & 9326572 & $44 \%+33 \% 1 \mathrm{~s}^{2} 2 \mathrm{p}^{2}\left({ }^{3} \mathrm{P}\right) 3 \mathrm{p}^{1}{ }^{4} \mathrm{D}+21 \% 1 \mathrm{~s}^{2} 2 \mathrm{p}^{2}\left({ }^{3} \mathrm{P}\right) 3 \mathrm{p}^{1{ }^{4} \mathrm{P}}$ \\
\hline 77 & $1 s^{2} 2 p^{2}\left({ }^{3} P\right) 3 p^{1}$ & ${ }^{4} \mathrm{P}$ & 1.5 & 9360271 & $32 \%+25 \% 1 \mathrm{~s}^{2} 2 \mathrm{p}^{2}\left({ }^{3} \mathrm{P}\right) 3 \mathrm{p}^{12} \mathrm{D}+20 \% 1 \mathrm{~s}^{2} 2 \mathrm{p}^{2}\left({ }^{3} \mathrm{P}\right) 3 \mathrm{p}^{1}{ }^{4} \mathrm{D}$ \\
\hline 78 & $1 \mathrm{~s}^{2} 2 \mathrm{p}^{2}\left({ }^{3} \mathrm{P}\right) 3 \mathrm{p}^{1}$ & ${ }^{4} \mathrm{D}$ & 2.5 & 9369611 & $86 \%$ \\
\hline 79 & $1 s^{2} 2 p^{2}\left({ }^{3} P\right) 3 p^{1}$ & ${ }^{4} \mathrm{P}$ & 0.5 & 9385693 & $64 \%+21 \% 1 \mathrm{~s}^{2} 2 \mathrm{p}^{2}\left({ }^{3} \mathrm{P}\right) 3 \mathrm{p}^{12} \mathrm{~S}$ \\
\hline 80 & $1 \mathrm{~s}^{2} 2 \mathrm{p}^{2}\left({ }^{1} \mathrm{D}\right) 3 \mathrm{~s}^{1}$ & ${ }^{2} \mathrm{D}$ & 2.5 & 9386490 & $77 \%+18 \% 1 \mathrm{~s}^{2} 2 \mathrm{p}^{2}\left({ }^{3} \mathrm{P}\right) 3 \mathrm{~s}^{1}{ }^{4} \mathrm{P}$ \\
\hline 81 & $1 \mathrm{~s}^{2} 2 \mathrm{p}^{2}\left({ }^{3} \mathrm{P}\right) 3 \mathrm{p}^{1}$ & ${ }^{4} \mathrm{P}$ & 2.5 & 9390309 & $43 \%+23 \% 1 \mathrm{~s}^{2} 2 \mathrm{p}^{2}\left({ }^{3} \mathrm{P}\right) 3 \mathrm{p}^{12} \mathrm{D}+20 \% 1 \mathrm{~s}^{2} 2 \mathrm{p}^{2}\left({ }^{1} \mathrm{D}\right) 3 \mathrm{p}^{12} \mathrm{D}$ \\
\hline 82 & $1 \mathrm{~s}^{2} 2 \mathrm{p}^{2}\left({ }^{3} \mathrm{P}\right) 3 \mathrm{p}^{1}$ & ${ }^{2} \mathrm{D}$ & 1.5 & 9405884 & $43 \%+32 \% 1 \mathrm{~s}^{2} 2 \mathrm{p}^{2}\left({ }^{3} \mathrm{P}\right) 3 \mathrm{p}^{1}{ }^{4} \mathrm{P}+14 \% 1 \mathrm{~s}^{2} 2 \mathrm{p}^{2}\left({ }^{1} \mathrm{D}\right) 3 \mathrm{p}^{1}{ }^{2} \mathrm{D}$ \\
\hline 83 & $1 \mathrm{~s}^{2} 2 \mathrm{p}^{2}\left({ }^{1} \mathrm{D}\right) 3 \mathrm{~s}^{1}$ & ${ }^{2} \mathrm{D}$ & 1.5 & 9406759 & $53 \%+37 \% 1 \mathrm{~s}^{2} 2 \mathrm{p}^{2}\left({ }^{3} \mathrm{P}\right) 3 \mathrm{~s}^{12} \mathrm{P}$ \\
\hline 84 & $1 \mathrm{~s}^{2} 2 \mathrm{p}^{2}\left({ }^{3} \mathrm{P}\right) 3 \mathrm{p}^{1}$ & ${ }^{4} \mathrm{D}$ & 3.5 & 9414721 & $80 \%+17 \% 1 \mathrm{~s}^{2} 2 \mathrm{p}^{2}\left({ }^{1} \mathrm{D}\right) 3 \mathrm{p}^{1}{ }^{2} \mathrm{~F}$ \\
\hline 85 & $1 \mathrm{~s}^{2} 2 \mathrm{p}^{2}\left({ }^{3} \mathrm{P}\right) 3 \mathrm{~d}^{1}$ & ${ }^{4} \mathrm{~F}$ & 1.5 & 9442168 & $70 \%+11 \% 1 \mathrm{~s}^{2} 2 \mathrm{p}^{2}\left({ }^{3} \mathrm{P}\right) 3 \mathrm{~d}^{14} \mathrm{D}$ \\
\hline 86 & $1 \mathrm{~s}^{2} 2 \mathrm{p}^{2}\left({ }^{3} \mathrm{P}\right) 3 \mathrm{p}^{1}$ & ${ }^{4} S$ & 1.5 & 9460642 & $53 \%+30 \% 1 \mathrm{~s}^{2} 2 \mathrm{p}^{2}\left({ }^{1} \mathrm{D}\right) 3 \mathrm{p}^{12} \mathrm{P}+13 \% 1 \mathrm{~s}^{2} 2 \mathrm{p}^{2}\left({ }^{3} \mathrm{P}\right) 3 \mathrm{p}^{12} \mathrm{P}$ \\
\hline 87 & $1 \mathrm{~s}^{2} 2 \mathrm{p}^{2}\left({ }^{3} \mathrm{P}\right) 3 \mathrm{~d}^{1}$ & ${ }^{4} \mathrm{~F}$ & 2.5 & 9466629 & $58 \%+24 \% 1 \mathrm{~s}^{2} 2 \mathrm{p}^{2}\left({ }^{3} \mathrm{P}\right) 3 \mathrm{~d}^{14} \mathrm{D}$ \\
\hline 88 & $1 \mathrm{~s}^{2} 2 \mathrm{p}^{2}\left({ }^{3} \mathrm{P}\right) 3 \mathrm{p}^{1}$ & ${ }^{4} \mathrm{P}$ & 2.5 & 9472291 & $34 \%+29 \% 1 \mathrm{~s}^{2} 2 \mathrm{p}^{2}\left({ }^{3} \mathrm{P}\right) 3 \mathrm{p}^{12} \mathrm{D}+29 \% 1 \mathrm{~s}^{2} 2 \mathrm{p}^{2}\left({ }^{1} \mathrm{D}\right) 3 \mathrm{p}^{12} \mathrm{~F}$ \\
\hline 89 & $1 \mathrm{~s}^{2} 2 \mathrm{p}^{2}\left({ }^{3} \mathrm{P}\right) 3 \mathrm{p}^{1}$ & ${ }^{2} \mathrm{P}$ & 1.5 & 9472381 & $47 \%+23 \% 1 \mathrm{~s}^{2} 2 \mathrm{p}^{2}\left({ }^{3} \mathrm{P}\right) 3 \mathrm{p}^{1}{ }^{4} \mathrm{~S}+11 \% 1 \mathrm{~s}^{2} 2 \mathrm{p}^{2}\left({ }^{3} \mathrm{P}\right) 3 \mathrm{p}^{1}{ }^{4} \mathrm{P}$ \\
\hline 90 & $1 s^{2} 2 p^{2}\left({ }^{3} \mathrm{P}\right) 3 p^{1}$ & ${ }^{2} \mathrm{P}$ & 0.5 & 9504310 & $69 \%+15 \% 1 \mathrm{~s}^{2} 2 \mathrm{p}^{2}\left({ }^{3} \mathrm{P}\right) 3 \mathrm{p}^{12} \mathrm{~S}$ \\
\hline 91 & $1 \mathrm{~s}^{2} 2 \mathrm{p}^{2}\left({ }^{3} \mathrm{P}\right) 3 \mathrm{~d}^{1}$ & ${ }^{4} \mathrm{~F}$ & 3.5 & 9514316 & $75 \%+21 \% 1 \mathrm{~s}^{2} 2 \mathrm{p}^{2}\left({ }^{3} \mathrm{P}\right) 3 \mathrm{~d}^{14} \mathrm{D}$ \\
\hline 92 & $1 \mathrm{~s}^{2} 2 \mathrm{p}^{2}\left({ }^{3} \mathrm{P}\right) 3 \mathrm{~d}^{1}$ & ${ }^{2} \mathrm{P}$ & 1.5 & 9516554 & $42 \%+25 \% 1 \mathrm{~s}^{2} 2 \mathrm{p}^{2}\left({ }^{3} \mathrm{P}\right) 3 \mathrm{~d}^{14} \mathrm{D}+23 \% 1 \mathrm{~s}^{2} 2 \mathrm{p}^{2}\left({ }^{3} \mathrm{P}\right) 3 \mathrm{~d}^{1{ }^{4}} \mathrm{~F}$ \\
\hline 93 & $1 \mathrm{~s}^{2} 2 \mathrm{p}^{2}\left({ }^{3} \mathrm{P}\right) 3 \mathrm{~d}^{1}$ & ${ }^{4} \mathrm{~F}$ & 2.5 & 9522857 & $32 \%+26 \% 1 \mathrm{~s}^{2} 2 \mathrm{p}^{2}\left({ }^{3} \mathrm{P}\right) 3 \mathrm{~d}^{1}{ }^{2} \mathrm{~F}+20 \% 1 \mathrm{~s}^{2} 2 \mathrm{p}^{2}\left({ }^{1} \mathrm{D}\right) 3 \mathrm{~d}^{1}{ }^{2} \mathrm{~F}$ \\
\hline 94 & $1 s^{2} 2 p^{2}\left({ }^{3} P\right) 3 d^{1}$ & ${ }^{4} \mathrm{D}$ & 0.5 & 9525480 & $83 \%+13 \% 1 \mathrm{~s}^{2} 2 \mathrm{p}^{2}\left({ }^{3} \mathrm{P}\right) 3 \mathrm{~d}^{12} \mathrm{P}$ \\
\hline 95 & $1 \mathrm{~s}^{2} 2 \mathrm{p}^{2}\left({ }^{1} \mathrm{D}\right) 3 \mathrm{p}^{1}$ & ${ }^{2} \mathrm{~F}$ & 2.5 & 9549139 & $49 \%+24 \% 1 \mathrm{~s}^{2} 2 \mathrm{p}^{2}\left({ }^{1} \mathrm{D}\right) 3 \mathrm{p}^{12} \mathrm{D}+15 \% 1 \mathrm{~s}^{2} 2 \mathrm{p}^{2}\left({ }^{3} \mathrm{P}\right) 3 \mathrm{p}^{12} \mathrm{D}$ \\
\hline 96 & $1 \mathrm{~s}^{2} 2 \mathrm{p}^{2}\left({ }^{3} \mathrm{P}\right) 3 \mathrm{~d}^{1}$ & ${ }^{4} \mathrm{D}$ & 3.5 & 9551033 & $38 \%+24 \% 1 \mathrm{~s}^{2} 2 \mathrm{p}^{2}\left({ }^{1} \mathrm{D}\right) 3 \mathrm{~d}^{12} \mathrm{~F}+16 \% 1 \mathrm{~s}^{2} 2 \mathrm{p}^{2}\left({ }^{3} \mathrm{P}\right) 3 \mathrm{~d}^{12} \mathrm{~F}$ \\
\hline 97 & $1 \mathrm{~s}^{2} 2 \mathrm{p}^{2}\left({ }^{3} \mathrm{P}\right) 3 \mathrm{~d}^{1}$ & ${ }^{4} \mathrm{~F}$ & 4.5 & 9552660 & $82 \%+18 \% 1 \mathrm{~s}^{2} 2 \mathrm{p}^{2}\left({ }^{1} \mathrm{D}\right) 3 \mathrm{~d}^{12} \mathrm{G}$ \\
\hline 98 & $1 \mathrm{~s}^{2} 2 \mathrm{p}^{2}\left({ }^{3} \mathrm{P}\right) 3 \mathrm{~d}^{1}$ & ${ }^{4} \mathrm{D}$ & 1.5 & 9555613 & $47 \%+33 \% 1 \mathrm{~s}^{2} 2 \mathrm{p}^{2}\left({ }^{3} \mathrm{P}\right) 3 \mathrm{~d}^{12} \mathrm{P}$ \\
\hline 99 & $1 \mathrm{~s}^{2} 2 \mathrm{p}^{2}\left({ }^{3} \mathrm{P}\right) 3 \mathrm{~d}^{1}$ & ${ }^{2} \mathrm{~F}$ & 2.5 & 9557712 & $32 \%+26 \% 1 \mathrm{~s}^{2} 2 \mathrm{p}^{2}\left({ }^{3} \mathrm{P}\right) 3 \mathrm{~d}^{14} \mathrm{P}+22 \% 1 \mathrm{~s}^{2} 2 \mathrm{p}^{2}\left({ }^{3} \mathrm{P}\right) 3 \mathrm{~d}^{1}{ }^{4} \mathrm{D}$ \\
\hline 100 & $1 \mathrm{~s}^{2} 2 \mathrm{p}^{2}\left({ }^{1} \mathrm{D}\right) 3 \mathrm{p}^{1}$ & ${ }^{2} \mathrm{~F}$ & 3.5 & 9563994 & $78 \%+19 \% 1 \mathrm{~s}^{2} 2 \mathrm{p}^{2}\left({ }^{3} \mathrm{P}\right) 3 \mathrm{p}^{1}{ }^{4} \mathrm{D}$ \\
\hline 101 & $1 s^{2} 2 p^{2}\left({ }^{1} D\right) 3 p^{1}$ & ${ }^{2} \mathrm{D}$ & 1.5 & 9583303 & $48 \%+22 \% 1 \mathrm{~s}^{2} 2 \mathrm{p}^{2}\left({ }^{1} \mathrm{D}\right) 3 \mathrm{p}^{12} \mathrm{P}+14 \% 1 \mathrm{~s}^{2} 2 \mathrm{p}^{2}\left({ }^{3} \mathrm{P}\right) 3 \mathrm{p}^{12} \mathrm{D}$ \\
\hline 102 & $1 \mathrm{~s}^{2} 2 \mathrm{p}^{2}\left({ }^{1} \mathrm{D}\right) 3 \mathrm{p}^{1}$ & ${ }^{2} \mathrm{D}$ & 2.5 & 9597713 & $55 \%+31 \% 1 \mathrm{~s}^{2} 2 \mathrm{p}^{2}\left({ }^{3} \mathrm{P}\right) 3 \mathrm{p}^{12} \mathrm{D}$ \\
\hline 103 & $1 \mathrm{~s}^{2} 2 \mathrm{p}^{2}\left({ }^{3} \mathrm{P}\right) 3 \mathrm{~d}^{1}$ & ${ }^{4} \mathrm{P}$ & 2.5 & 9599924 & $48 \%+31 \% 1 \mathrm{~s}^{2} 2 \mathrm{p}^{2}\left({ }^{3} \mathrm{P}\right) 3 \mathrm{~d}^{14} \mathrm{D}+16 \% 1 \mathrm{~s}^{2} 2 \mathrm{p}^{2}\left({ }^{1} \mathrm{D}\right) 3 \mathrm{~d}^{12} \mathrm{D}$ \\
\hline 104 & $1 s^{2} 2 p^{2}\left({ }^{1} S\right) 3 s^{1}$ & ${ }^{2} \mathrm{~S}$ & 0.5 & 9602844 & $59 \%+13 \% 1 \mathrm{~s}^{2} 2 \mathrm{p}^{2}\left({ }^{3} \mathrm{P}\right) 3 \mathrm{~d}^{1}{ }^{2} \mathrm{P}+11 \% 1 \mathrm{~s}^{2} 2 \mathrm{p}^{2}\left({ }^{1} \mathrm{D}\right) 3 \mathrm{~d}^{1}{ }^{2} \mathrm{~S}$ \\
\hline 105 & $1 s^{2} 2 p^{2}\left({ }^{1} \mathrm{D}\right) 3 p^{1}$ & ${ }^{2} \mathrm{P}$ & 0.5 & 9611964 & $89 \%$ \\
\hline 106 & $1 \mathrm{~s}^{2} 2 \mathrm{p}^{2}\left({ }^{3} \mathrm{P}\right) 3 \mathrm{~d}^{1}$ & ${ }^{4} \mathrm{P}$ & 1.5 & 9613678 & $74 \%$ \\
\hline 107 & $1 \mathrm{~s}^{2} 2 \mathrm{p}^{2}\left({ }^{3} \mathrm{P}\right) 3 \mathrm{~d}^{1}$ & ${ }^{2} \mathrm{P}$ & 0.5 & 9621528 & $49 \%+24 \% 1 \mathrm{~s}^{2} 2 \mathrm{p}^{2}\left({ }^{1} \mathrm{~S}\right) 3 \mathrm{~s}^{12} \mathrm{~S}+16 \% 1 \mathrm{~s}^{2} 2 \mathrm{p}^{2}\left({ }^{3} \mathrm{P}\right) 3 \mathrm{~d}^{1}{ }^{4} \mathrm{P}$ \\
\hline 108 & $1 \mathrm{~s}^{2} 2 \mathrm{p}^{2}\left({ }^{3} \mathrm{P}\right) 3 \mathrm{~d}^{1}$ & ${ }^{4} \mathrm{P}$ & 0.5 & 9624060 & $63 \%+21 \% 1 \mathrm{~s}^{2} 2 \mathrm{p}^{2}\left({ }^{3} \mathrm{P}\right) 3 \mathrm{~d}^{12} \mathrm{P}$ \\
\hline 109 & $1 \mathrm{~s}^{2} 2 \mathrm{p}^{2}\left({ }^{3} \mathrm{P}\right) 3 \mathrm{~d}^{1}$ & ${ }^{2} \mathrm{~F}$ & 3.5 & 9629317 & $36 \%+29 \% 1 \mathrm{~s}^{2} 2 \mathrm{p}^{2}\left({ }^{1} \mathrm{D}\right) 3 \mathrm{~d}^{12} \mathrm{G}+28 \% 1 \mathrm{~s}^{2} 2 \mathrm{p}^{2}\left({ }^{3} \mathrm{P}\right) 3 \mathrm{~d}^{14} \mathrm{D}$ \\
\hline 110 & $1 \mathrm{~s}^{2} 2 \mathrm{p}^{2}\left({ }^{3} \mathrm{P}\right) 3 \mathrm{~d}^{1}$ & ${ }^{2} \mathrm{D}$ & 2.5 & 9667354 & $60 \%+16 \% 1 \mathrm{~s}^{2} 2 \mathrm{p}^{2}\left({ }^{1} \mathrm{D}\right) 3 \mathrm{~d}^{1}{ }^{2} \mathrm{~F}+12 \% 1 \mathrm{~s}^{2} 2 \mathrm{p}^{2}\left({ }^{1} \mathrm{D}\right) 3 \mathrm{~d}^{12} \mathrm{D}$ \\
\hline 111 & $1 \mathrm{~s}^{2} 2 \mathrm{p}^{2}\left({ }^{3} \mathrm{P}\right) 3 \mathrm{~d}^{1}$ & ${ }^{2} \mathrm{D}$ & 1.5 & 9670700 & $71 \%+12 \% 1 \mathrm{~s}^{2} 2 \mathrm{p}^{2}\left({ }^{1} \mathrm{D}\right) 3 \mathrm{~d}^{12} \mathrm{D}$ \\
\hline 112 & $1 \mathrm{~s}^{2} 2 \mathrm{p}^{2}\left({ }^{1} \mathrm{D}\right) 3 \mathrm{p}^{1}$ & ${ }^{2} \mathrm{P}$ & 1.5 & 9683971 & $39 \%+32 \% 1 \mathrm{~s}^{2} 2 \mathrm{p}^{2}\left({ }^{3} \mathrm{P}\right) 3 \mathrm{p}^{1}{ }^{2} \mathrm{P}+14 \% 1 \mathrm{~s}^{2} 2 \mathrm{p}^{2}\left({ }^{1} \mathrm{D}\right) 3 \mathrm{p}^{1{ }^{2} \mathrm{D}}$ \\
\hline 113 & $1 \mathrm{~s}^{2} 2 \mathrm{p}^{2}\left({ }^{1} \mathrm{D}\right) 3 \mathrm{~d}^{1}$ & ${ }^{2} \mathrm{G}$ & 3.5 & 9691641 & $45 \%+39 \% 1 \mathrm{~s}^{2} 2 \mathrm{p}^{2}\left({ }^{1} \mathrm{D}\right) 3 \mathrm{~d}^{12} \mathrm{~F}+11 \% 1 \mathrm{~s}^{2} 2 \mathrm{p}^{2}\left({ }^{3} \mathrm{P}\right) 3 \mathrm{~d}^{14} \mathrm{D}$ \\
\hline 114 & $1 \mathrm{~s}^{2} 2 \mathrm{p}^{2}\left({ }^{1} \mathrm{D}\right) 3 \mathrm{~d}^{1}$ & ${ }^{2} \mathrm{G}$ & 4.5 & 9708418 & $82 \%+18 \% 1 \mathrm{~s}^{2} 2 \mathrm{p}^{2}\left({ }^{3} \mathrm{P}\right) 3 \mathrm{~d}^{14} \mathrm{~F}$ \\
\hline 115 & $1 \mathrm{~s}^{2} 2 \mathrm{p}^{2}\left({ }^{1} \mathrm{D}\right) 3 \mathrm{~d}^{1}$ & ${ }^{2} \mathrm{D}$ & 1.5 & 9736179 & $73 \%$ \\
\hline 116 & $1 \mathrm{~s}^{2} 2 \mathrm{p}^{2}\left({ }^{1} \mathrm{D}\right) 3 \mathrm{~d}^{1}$ & ${ }^{2} \mathrm{D}$ & 2.5 & 9740434 & $52 \%+23 \% 1 \mathrm{~s}^{2} 2 \mathrm{p}^{2}\left({ }^{1} \mathrm{D}\right) 3 \mathrm{~d}^{12} \mathrm{~F}$ \\
\hline 117 & $1 \mathrm{~s}^{2} 2 \mathrm{p}^{2}\left({ }^{3} \mathrm{P}\right) 3 \mathrm{~d}^{1}$ & ${ }^{2} \mathrm{~F}$ & 3.5 & 9762003 & $40 \%+36 \% 1 \mathrm{~s}^{2} 2 \mathrm{p}^{2}\left({ }^{1} \mathrm{D}\right) 3 \mathrm{~d}^{12} \mathrm{~F}+17 \% 1 \mathrm{~s}^{2} 2 \mathrm{p}^{2}\left({ }^{1} \mathrm{D}\right) 3 \mathrm{~d}^{12} \mathrm{G}$ \\
\hline 118 & $1 \mathrm{~s}^{2} 2 \mathrm{p}^{2}\left({ }^{1} \mathrm{D}\right) 3 \mathrm{~d}^{1}$ & ${ }^{2} \mathrm{P}$ & 0.5 & 9763792 & $89 \%$ \\
\hline 119 & $1 \mathrm{~s}^{2} 2 \mathrm{p}^{2}\left({ }^{1} \mathrm{~S}\right) 3 \mathrm{p}^{1}$ & ${ }^{2} \mathrm{P}$ & 0.5 & 9792303 & $84 \%$ \\
\hline
\end{tabular}


Table 1. continued.

\begin{tabular}{|c|c|c|c|c|c|}
\hline Index & Configuration & $L S$ & $J$ & $E\left(\mathrm{~cm}^{-1}\right)$ & Composition \\
\hline 120 & $1 \mathrm{~s}^{2} 2 \mathrm{p}^{2}\left({ }^{3} \mathrm{P}\right) 3 \mathrm{~d}^{1}$ & ${ }^{2} \mathrm{D}$ & 2.5 & 9801608 & $36 \%+27 \% 1 \mathrm{~s}^{2} 2 \mathrm{p}^{2}\left({ }^{1} \mathrm{D}\right) 3 \mathrm{~d}^{12} \mathrm{~F}+18 \% 1 \mathrm{~s}^{2} 2 \mathrm{p}^{2}\left({ }^{1} \mathrm{D}\right) 3 \mathrm{~d}^{12} \mathrm{D}$ \\
\hline 121 & $1 \mathrm{~s}^{2} 2 \mathrm{p}^{2}\left({ }^{1} \mathrm{D}\right) 3 \mathrm{~d}^{1}$ & ${ }^{2} \mathrm{~S}$ & 0.5 & 9804009 & $80 \%$ \\
\hline 122 & $1 \mathrm{~s}^{2} 2 \mathrm{p}^{2}\left({ }^{1} \mathrm{D}\right) 3 \mathrm{~d}^{1}$ & ${ }^{2} \mathrm{P}$ & 1.5 & 9806502 & $69 \%+12 \% 1 \mathrm{~s}^{2} 2 \mathrm{p}^{2}\left({ }^{3} \mathrm{P}\right) 3 \mathrm{~d}^{12} \mathrm{P}$ \\
\hline 123 & $1 s^{2} 2 p^{2}\left({ }^{1} S\right) 3 p^{1}$ & ${ }^{2} \mathrm{P}$ & 1.5 & 9810603 & $86 \%$ \\
\hline 124 & $1 \mathrm{~s}^{2} 2 \mathrm{p}^{2}\left({ }^{1} \mathrm{~S}\right) 3 \mathrm{~d}^{1}$ & ${ }^{2} \mathrm{D}$ & 2.5 & 9960890 & $87 \%$ \\
\hline 125 & $1 \mathrm{~s}^{2} 2 \mathrm{p}^{2}\left({ }^{1} \mathrm{~S}\right) 3 \mathrm{~d}^{1}$ & ${ }^{2} \mathrm{D}$ & 1.5 & 9969426 & $83 \%$ \\
\hline 126 & $1 s^{2} 2 s^{2} 4 s^{1}$ & ${ }^{2} \mathrm{~S}$ & 0.5 & 10986106 & $95 \%$ \\
\hline 127 & $1 s^{2} 2 s^{2} 4 p^{1}$ & ${ }^{2} \mathrm{P}$ & 0.5 & 11061237 & $95 \%$ \\
\hline 128 & $1 s^{2} 2 s^{2} 4 p^{1}$ & ${ }^{2} \mathrm{P}$ & 1.5 & 11075121 & $95 \%$ \\
\hline 129 & $1 s^{2} 2 s^{2} 4 d^{1}$ & ${ }^{2} \mathrm{D}$ & 1.5 & 11135585 & $95 \%$ \\
\hline 130 & $1 s^{2} 2 s^{2} 4 d^{1}$ & ${ }^{2} \mathrm{D}$ & 2.5 & 11139649 & $95 \%$ \\
\hline 131 & $1 s^{2} 2 s^{2} 4 f^{1}$ & ${ }^{2} \mathrm{~F}$ & 2.5 & 11173539 & $95 \%$ \\
\hline 132 & $1 s^{2} 2 s^{2} 4 f^{1}$ & ${ }^{2} \mathrm{~F}$ & 3.5 & 11175356 & $95 \%$ \\
\hline 133 & $1 s^{2} 2 s^{1} 2 p^{1}\left({ }^{3} P\right) 4 s^{1}$ & ${ }^{4} \mathrm{P}$ & 0.5 & 11340530 & $88 \%+11 \% 1 \mathrm{~s}^{2} 2 \mathrm{~s}^{1} 2 \mathrm{p}^{1}\left({ }^{3} \mathrm{P}\right) 4 \mathrm{~s}^{1}{ }^{2} \mathrm{P}$ \\
\hline 134 & $1 s^{2} 2 s^{1} 2 p^{1}\left({ }^{3} P\right) 4 s^{1}$ & ${ }^{4} \mathrm{P}$ & 1.5 & 11369374 & $86 \%+11 \% 1 \mathrm{~s}^{2} 2 \mathrm{~s}^{1} 2 \mathrm{p}^{1}\left({ }^{3} \mathrm{P}\right) 4 \mathrm{~s}^{1}{ }^{2} \mathrm{P}$ \\
\hline 135 & $1 s^{2} 2 s^{1} 2 p^{1}\left({ }^{3} P\right) 4 s^{1}$ & ${ }^{2} \mathrm{P}$ & 0.5 & 11389750 & $87 \%+10 \% 1 \mathrm{~s}^{2} 2 \mathrm{~s}^{1} 2 \mathrm{p}^{1}\left({ }^{3} \mathrm{P}\right) 4 \mathrm{~s}^{14} \mathrm{P}$ \\
\hline 136 & $1 s^{2} 2 s^{1} 2 p^{1}\left({ }^{3} P\right) 4 p^{1}$ & ${ }^{4} \mathrm{D}$ & 0.5 & 11407650 & $74 \%+19 \% 1 \mathrm{~s}^{2} 2 \mathrm{~s}^{1} 2 \mathrm{p}^{1}\left({ }^{3} \mathrm{P}\right) 4 \mathrm{p}^{1}{ }^{2} \mathrm{P}$ \\
\hline 137 & $1 s^{2} 2 s^{1} 2 p^{1}\left({ }^{3} P\right) 4 p^{1}$ & ${ }^{4} \mathrm{D}$ & 1.5 & 11428039 & $69 \%+20 \% 1 \mathrm{~s}^{2} 2 \mathrm{~s}^{1} 2 \mathrm{p}^{1}\left({ }^{3} \mathrm{P}\right) 4 \mathrm{p}^{14} \mathrm{P}$ \\
\hline 138 & $1 s^{2} 2 s^{1} 2 p^{1}\left({ }^{3} P\right) 4 p^{1}$ & ${ }^{2} \mathrm{P}$ & 1.5 & 11439838 & $33 \%+22 \% 1 \mathrm{~s}^{2} 2 \mathrm{~s}^{1} 2 \mathrm{p}^{1}\left({ }^{3} \mathrm{P}\right) 4 \mathrm{p}^{1}{ }^{4} \mathrm{~S}+17 \% 1 \mathrm{~s}^{2} 2 \mathrm{~s}^{1} 2 \mathrm{p}^{1}\left({ }^{3} \mathrm{P}\right) 4 \mathrm{p}^{1{ }^{4} \mathrm{D}}$ \\
\hline 139 & $1 s^{2} 2 s^{1} 2 p^{1}\left({ }^{3} P\right) 4 s^{1}$ & ${ }^{4} \mathrm{P}$ & 2.5 & 11457080 & $97 \%$ \\
\hline 140 & $1 s^{2} 2 s^{1} 2 p^{1}\left({ }^{3} P\right) 4 p^{1}$ & ${ }^{4} \mathrm{P}$ & 0.5 & 11457418 & $81 \%+13 \% 1 \mathrm{~s}^{2} 2 \mathrm{~s}^{1} 2 \mathrm{p}^{1}\left({ }^{3} \mathrm{P}\right) 4 \mathrm{p}^{12} \mathrm{P}$ \\
\hline 141 & $1 s^{2} 2 s^{1} 2 p^{1}\left({ }^{3} P\right) 4 p^{1}$ & ${ }^{4} \mathrm{D}$ & 2.5 & 11457419 & $70 \%+19 \% 1 \mathrm{~s}^{2} 2 \mathrm{~s}^{1} 2 \mathrm{p}^{1}\left({ }^{3} \mathrm{P}\right) 4 \mathrm{p}^{1}{ }^{4} \mathrm{P}$ \\
\hline 142 & $1 s^{2} 2 s^{1} 2 p^{1}\left({ }^{3} P\right) 4 p^{1}$ & ${ }^{2} \mathrm{P}$ & 0.5 & 11460571 & $49 \%+23 \% 1 s^{2} 2 s^{1} 2 p^{1}\left({ }^{3} P\right) 4 p^{14} D+21 \% 1 s^{2} 2 s^{1} 2 p^{1}\left({ }^{3} P\right) 4 p^{12} S$ \\
\hline 143 & $1 s^{2} 2 s^{1} 2 p^{1}\left({ }^{3} P\right) 4 p^{1}$ & ${ }^{2} \mathrm{D}$ & 1.5 & 11470801 & $57 \%+19 \% 1 \mathrm{~s}^{2} 2 \mathrm{~s}^{1} 2 \mathrm{p}^{1}\left({ }^{3} \mathrm{P}\right) 4 \mathrm{p}^{1}{ }^{4} \mathrm{~S}+16 \% 1 \mathrm{~s}^{2} 2 \mathrm{~s}^{1} 2 \mathrm{p}^{1}\left({ }^{3} \mathrm{P}\right) 4 \mathrm{p}^{1}{ }^{4} \mathrm{P}$ \\
\hline 144 & $1 s^{2} 2 s^{1} 2 p^{1}\left({ }^{3} P\right) 4 d^{1}$ & ${ }^{4} \mathrm{~F}$ & 1.5 & 11478339 & $76 \%+10 \% 1 \mathrm{~s}^{2} 2 \mathrm{~s}^{1} 2 \mathrm{p}^{1}\left({ }^{3} \mathrm{P}\right) 4 \mathrm{~d}^{1}{ }^{4} \mathrm{D}$ \\
\hline 145 & $1 s^{2} 2 s^{1} 2 p^{1}\left({ }^{3} P\right) 4 s^{1}$ & ${ }^{2} \mathrm{P}$ & 1.5 & 11481674 & $88 \%+11 \% 1 \mathrm{~s}^{2} 2 \mathrm{~s}^{1} 2 \mathrm{p}^{1}\left({ }^{3} \mathrm{P}\right) 4 \mathrm{~s}^{1}{ }^{4} \mathrm{P}$ \\
\hline 146 & $1 s^{2} 2 s^{1} 2 p^{1}\left({ }^{3} P\right) 4 d^{1}$ & ${ }^{4} \mathrm{~F}$ & 2.5 & 11489897 & $51 \%+28 \% 1 \mathrm{~s}^{2} 2 \mathrm{~s}^{1} 2 \mathrm{p}^{1}\left({ }^{3} \mathrm{P}\right) 4 \mathrm{~d}^{14} \mathrm{D}+12 \% 1 \mathrm{~s}^{2} 2 \mathrm{~s}^{1} 2 \mathrm{p}^{1}\left({ }^{3} \mathrm{P}\right) 4 \mathrm{~d}^{1}{ }^{4} \mathrm{P}$ \\
\hline 147 & $1 s^{2} 2 s^{1} 2 p^{1}\left({ }^{3} P\right) 4 d^{1}$ & ${ }^{4} \mathrm{P}$ & 2.5 & 11511057 & $33 \%+31 \% 1 \mathrm{~s}^{2} 2 \mathrm{~s}^{1} 2 \mathrm{p}^{1}\left({ }^{3} \mathrm{P}\right) 4 \mathrm{~d}^{14} \mathrm{~F}+26 \% 1 \mathrm{~s}^{2} 2 \mathrm{~s}^{1} 2 \mathrm{p}^{1}\left({ }^{3} \mathrm{P}\right) 4 \mathrm{~d}^{1}{ }^{2} \mathrm{D}$ \\
\hline 148 & $1 s^{2} 2 s^{1} 2 p^{1}\left({ }^{3} P\right) 4 d^{1}$ & ${ }^{4} \mathrm{~F}$ & 3.5 & 11516474 & $62 \%+29 \% 1 \mathrm{~s}^{2} 2 \mathrm{~s}^{1} 2 \mathrm{p}^{1}\left({ }^{3} \mathrm{P}\right) 4 \mathrm{~d}^{14} \mathrm{D}$ \\
\hline 149 & $1 s^{2} 2 s^{1} 2 p^{1}\left({ }^{3} P\right) 4 d^{1}$ & ${ }^{4} \mathrm{D}$ & 1.5 & 11518812 & $50 \%+29 \% 1 \mathrm{~s}^{2} 2 \mathrm{~s}^{1} 2 \mathrm{p}^{1}\left({ }^{3} \mathrm{P}\right) 4 \mathrm{~d}^{1}{ }^{4} \mathrm{P}$ \\
\hline 150 & $1 s^{2} 2 s^{1} 2 p^{1}\left({ }^{3} P\right) 4 d^{1}$ & ${ }^{4} \mathrm{D}$ & 0.5 & 11521977 & $81 \%$ \\
\hline 151 & $1 \mathrm{~s}^{2} 2 \mathrm{~s}^{1} 2 \mathrm{p}^{1}\left({ }^{3} \mathrm{P}\right) 4 \mathrm{f}^{1}$ & ${ }^{4} \mathrm{G}$ & 2.5 & 11522349 & $48 \%+21 \% 1 s^{2} 2 s^{1} 2 p^{1}\left({ }^{3} P\right) 4 f^{1}{ }^{4} F+14 \% 1 s^{2} 2 s^{1} 2 p^{1}\left({ }^{3} P\right) 4 f^{1}{ }^{2} F$ \\
\hline 152 & $1 \mathrm{~s}^{2} 2 \mathrm{~s}^{1} 2 \mathrm{p}^{1}\left({ }^{3} \mathrm{P}\right) 4 \mathrm{f}^{1}$ & ${ }^{4} \mathrm{~F}$ & 3.5 & 11524662 & $30 \%+22 \% 1 \mathrm{~s}^{2} 2 \mathrm{~s}^{1} 2 \mathrm{p}^{1}\left({ }^{3} \mathrm{P}\right) 4 \mathrm{f}^{1}{ }^{4} \mathrm{D}+20 \% 1 \mathrm{~s}^{2} 2 \mathrm{~s}^{1} 2 \mathrm{p}^{1}\left({ }^{3} \mathrm{P}\right) 4 \mathrm{f}^{1}{ }^{4} \mathrm{G}$ \\
\hline 153 & $1 s^{2} 2 s^{1} 2 p^{1}\left({ }^{3} P\right) 4 d^{1}$ & ${ }^{2} \mathrm{D}$ & 1.5 & 11534686 & $50 \%+34 \% 1 \mathrm{~s}^{2} 2 \mathrm{~s}^{1} 2 \mathrm{p}^{1}\left({ }^{3} \mathrm{P}\right) 4 \mathrm{~d}^{12} \mathrm{P}+13 \% 1 \mathrm{~s}^{2} 2 \mathrm{~s}^{1} 2 \mathrm{p}^{1}\left({ }^{3} \mathrm{P}\right) 4 \mathrm{~d}^{1{ }^{4} \mathrm{~F}}$ \\
\hline 154 & $1 s^{2} 2 s^{1} 2 p^{1}\left({ }^{3} P\right) 4 p^{1}$ & ${ }^{4} \mathrm{P}$ & 1.5 & 11538763 & $43 \%+23 \% 1 s^{2} 2 s^{1} 2 p^{1}\left({ }^{3} \mathrm{P}\right) 4 \mathrm{p}^{12} \mathrm{P}+16 \% 1 \mathrm{~s}^{2} 2 \mathrm{~s}^{1} 2 \mathrm{p}^{1}\left({ }^{3} \mathrm{P}\right) 4 \mathrm{p}^{1}{ }^{4} \mathrm{~S}$ \\
\hline 155 & $1 s^{2} 2 s^{1} 2 p^{1}\left({ }^{3} P\right) 4 p^{1}$ & ${ }^{4} \mathrm{D}$ & 3.5 & 11539955 & $99 \%$ \\
\hline 156 & $1 s^{2} 2 s^{1} 2 p^{1}\left({ }^{3} P\right) 4 d^{1}$ & ${ }^{2} \mathrm{~F}$ & 2.5 & 11541402 & $59 \%+15 \% 1 s^{2} 2 s^{1} 2 p^{1}\left({ }^{3} P\right) 4 d^{14} P+15 \% 1 s^{2} 2 s^{1} 2 p^{1}\left({ }^{3} P\right) 4 d^{12} D$ \\
\hline 157 & $1 s^{2} 2 s^{1} 2 p^{1}\left({ }^{3} P\right) 4 p^{1}$ & ${ }^{4} \mathrm{P}$ & 2.5 & 11542484 & $52 \%+28 \% 1 s^{2} 2 s^{1} 2 p^{1}\left({ }^{3} P\right) 4 p^{14} D+19 \% 1 s^{2} 2 s^{1} 2 p^{1}\left({ }^{3} P\right) 4 p^{12} D$ \\
\hline 158 & $1 s^{2} 2 s^{1} 2 p^{1}\left({ }^{3} P\right) 4 p^{1}$ & ${ }^{2} \mathrm{P}$ & 1.5 & 11549509 & $35 \%+33 \% 1 s^{2} 2 s^{1} 2 p^{1}\left({ }^{3} \mathrm{P}\right) 4 p^{14} \mathrm{~S}+12 \% 1 \mathrm{~s}^{2} 2 \mathrm{~s}^{1} 2 \mathrm{p}^{1}\left({ }^{3} \mathrm{P}\right) 4 \mathrm{p}^{1}{ }^{2} \mathrm{D}$ \\
\hline 159 & $1 \mathrm{~s}^{2} 2 \mathrm{~s}^{1} 2 \mathrm{p}^{1}\left({ }^{3} \mathrm{P}\right) 4 \mathrm{f}^{1}$ & ${ }^{4} \mathrm{~F}$ & 1.5 & 11551577 & $63 \%+12 \% 1 \mathrm{~s}^{2} 2 \mathrm{~s}^{1} 2 \mathrm{p}^{1}\left({ }^{3} \mathrm{P}\right) 4 \mathrm{f}^{14} \mathrm{D}+11 \% 1 \mathrm{~s}^{2} 2 \mathrm{~s}^{1} 2 \mathrm{p}^{1}\left({ }^{3} \mathrm{P}\right) 4 \mathrm{f}^{12} \mathrm{D}$ \\
\hline 160 & $1 \mathrm{~s}^{2} 2 \mathrm{~s}^{1} 2 \mathrm{p}^{1}\left({ }^{3} \mathrm{P}\right) 4 \mathrm{f}^{1}$ & ${ }^{4} \mathrm{G}$ & 3.5 & 11552614 & $44 \%+33 \% 1 \mathrm{~s}^{2} 2 \mathrm{~s}^{1} 2 \mathrm{p}^{1}\left({ }^{3} \mathrm{P}\right) 4 \mathrm{f}^{12} \mathrm{~F}+11 \% 1 \mathrm{~s}^{2} 2 \mathrm{~s}^{1} 2 \mathrm{p}^{1}\left({ }^{3} \mathrm{P}\right) 4 \mathrm{f}^{1}{ }^{2} \mathrm{G}$ \\
\hline 161 & $1 \mathrm{~s}^{2} 2 \mathrm{~s}^{1} 2 \mathrm{p}^{1}\left({ }^{3} \mathrm{P}\right) 4 \mathrm{f}^{1}$ & ${ }^{4} \mathrm{~F}$ & 2.5 & 11553334 & $34 \%+29 \% 1 \mathrm{~s}^{2} 2 \mathrm{~s}^{1} 2 \mathrm{p}^{1}\left({ }^{3} \mathrm{P}\right) 4 \mathrm{f}^{1}{ }^{4} \mathrm{D}+27 \% 1 \mathrm{~s}^{2} 2 \mathrm{~s}^{1} 2 \mathrm{p}^{1}\left({ }^{3} \mathrm{P}\right) 4 \mathrm{f}^{1}{ }^{2} \mathrm{~F}$ \\
\hline 162 & $1 \mathrm{~s}^{2} 2 \mathrm{~s}^{1} 2 \mathrm{p}^{1}\left({ }^{3} \mathrm{P}\right) 4 \mathrm{f}^{1}$ & ${ }^{4} \mathrm{G}$ & 4.5 & 11553358 & $43 \%+41 \% 1 \mathrm{~s}^{2} 2 \mathrm{~s}^{1} 2 \mathrm{p}^{1}\left({ }^{3} \mathrm{P}\right) 4 \mathrm{f}^{1}{ }^{4} \mathrm{~F}+14 \% 1 \mathrm{~s}^{2} 2 \mathrm{~s}^{1} 2 \mathrm{p}^{1}\left({ }^{3} \mathrm{P}\right) 4 \mathrm{f}^{12} \mathrm{G}$ \\
\hline 163 & $1 \mathrm{~s}^{2} 2 \mathrm{~s}^{1} 2 \mathrm{p}^{1}\left({ }^{3} \mathrm{P}\right) 4 \mathrm{f}^{1}$ & ${ }^{2} \mathrm{D}$ & 2.5 & 11558617 & $41 \%+34 \% 1 \mathrm{~s}^{2} 2 \mathrm{~s}^{1} 2 \mathrm{p}^{1}\left({ }^{3} \mathrm{P}\right) 4 \mathrm{f}^{1}{ }^{4} \mathrm{G}+17 \% 1 \mathrm{~s}^{2} 2 \mathrm{~s}^{1} 2 \mathrm{p}^{1}\left({ }^{3} \mathrm{P}\right) 4 \mathrm{f}^{1}{ }^{2} \mathrm{~F}$ \\
\hline 164 & $1 \mathrm{~s}^{2} 2 \mathrm{~s}^{1} 2 \mathrm{p}^{1}\left({ }^{3} \mathrm{P}\right) 4 \mathrm{f}^{1}$ & ${ }^{2} \mathrm{G}$ & 3.5 & 11559897 & $44 \%+38 \% 1 \mathrm{~s}^{2} 2 \mathrm{~s}^{1} 2 \mathrm{p}^{1}\left({ }^{3} \mathrm{P}\right) 4 \mathrm{f}^{14} \mathrm{D}$ \\
\hline 165 & $1 s^{2} 2 s^{1} 2 p^{1}\left({ }^{3} P\right) 4 p^{1}$ & ${ }^{2} \mathrm{D}$ & 2.5 & 11566890 & $71 \%+26 \% 1 \mathrm{~s}^{2} 2 \mathrm{~s}^{1} 2 \mathrm{p}^{1}\left({ }^{3} \mathrm{P}\right) 4 \mathrm{p}^{1}{ }^{4} \mathrm{P}$ \\
\hline 166 & $1 s^{2} 2 s^{1} 2 p^{1}\left({ }^{3} P\right) 4 p^{1}$ & ${ }^{2} \mathrm{~S}$ & 0.5 & 11577839 & $71 \%+16 \% 1 \mathrm{~s}^{2} 2 \mathrm{~s}^{1} 2 \mathrm{p}^{1}\left({ }^{3} \mathrm{P}\right) 4 \mathrm{p}^{12} \mathrm{P}$ \\
\hline 167 & $1 s^{2} 2 s^{1} 2 p^{1}\left({ }^{3} P\right) 4 d^{1}$ & ${ }^{4} \mathrm{~F}$ & 4.5 & 11596554 & $100 \%$ \\
\hline 168 & $1 s^{2} 2 s^{1} 2 p^{1}\left({ }^{3} P\right) 4 d^{1}$ & ${ }^{4} \mathrm{D}$ & 3.5 & 11604622 & $63 \%+35 \% 1 \mathrm{~s}^{2} 2 \mathrm{~s}^{1} 2 \mathrm{p}^{1}\left({ }^{3} \mathrm{P}\right) 4 \mathrm{~d}^{1}{ }^{4} \mathrm{~F}$ \\
\hline 169 & $1 s^{2} 2 s^{1} 2 p^{1}\left({ }^{3} P\right) 4 d^{1}$ & ${ }^{4} \mathrm{D}$ & 2.5 & 11607936 & $56 \%+20 \% 1 \mathrm{~s}^{2} 2 \mathrm{~s}^{1} 2 \mathrm{p}^{1}\left({ }^{3} \mathrm{P}\right) 4 \mathrm{~d}^{14} \mathrm{P}+14 \% 1 \mathrm{~s}^{2} 2 \mathrm{~s}^{1} 2 \mathrm{p}^{1}\left({ }^{3} \mathrm{P}\right) 4 \mathrm{~d}^{1{ }^{4} \mathrm{~F}}$ \\
\hline 170 & $1 s^{2} 2 s^{1} 2 p^{1}\left({ }^{3} P\right) 4 d^{1}$ & ${ }^{4} \mathrm{P}$ & 1.5 & 11611137 & $61 \%+36 \% 1 \mathrm{~s}^{2} 2 \mathrm{~s}^{1} 2 \mathrm{p}^{1}\left({ }^{3} \mathrm{P}\right) 4 \mathrm{~d}^{14} \mathrm{D}$ \\
\hline 171 & $1 s^{2} 2 s^{1} 2 p^{1}\left({ }^{3} P\right) 4 d^{1}$ & ${ }^{4} \mathrm{P}$ & 0.5 & 11612623 & $90 \%$ \\
\hline 172 & $1 s^{2} 2 s^{1} 2 p^{1}\left({ }^{3} P\right) 4 d^{1}$ & ${ }^{2} \mathrm{D}$ & 2.5 & 11620801 & $50 \%+25 \% 1 \mathrm{~s}^{2} 2 \mathrm{~s}^{1} 2 \mathrm{p}^{1}\left({ }^{3} \mathrm{P}\right) 4 \mathrm{~d}^{12} \mathrm{~F}+17 \% 1 \mathrm{~s}^{2} 2 \mathrm{~s}^{1} 2 \mathrm{p}^{1}\left({ }^{3} \mathrm{P}\right) 4 \mathrm{~d}^{1}{ }^{4} \mathrm{P}$ \\
\hline 173 & $1 s^{2} 2 s^{1} 2 p^{1}\left({ }^{3} P\right) 4 d^{1}$ & ${ }^{2} \mathrm{P}$ & 1.5 & 11632223 & $57 \%+29 \% 1 \mathrm{~s}^{2} 2 \mathrm{~s}^{1} 2 \mathrm{p}^{1}\left({ }^{3} \mathrm{P}\right) 4 \mathrm{~d}^{12} \mathrm{D}$ \\
\hline 174 & $1 s^{2} 2 s^{1} 2 p^{1}\left({ }^{3} P\right) 4 d^{1}$ & ${ }^{2} \mathrm{~F}$ & 3.5 & 11641117 & $91 \%$ \\
\hline 175 & $1 s^{2} 2 s^{1} 2 p^{1}\left({ }^{3} P\right) 4 f^{1}$ & ${ }^{4} \mathrm{~F}$ & 3.5 & 11642518 & $46 \%+26 \% 1 \mathrm{~s}^{2} 2 \mathrm{~s}^{1} 2 \mathrm{p}^{1}\left({ }^{3} \mathrm{P}\right) 4 \mathrm{f}^{14} \mathrm{G}+12 \% 1 \mathrm{~s}^{2} 2 \mathrm{~s}^{1} 2 \mathrm{p}^{1}\left({ }^{3} \mathrm{P}\right) 4 \mathrm{f}^{12} \mathrm{~F}$ \\
\hline 176 & $1 s^{2} 2 s^{1} 2 p^{1}\left({ }^{3} P\right) 4 f^{1}$ & ${ }^{4} \mathrm{~F}$ & 4.5 & 11642764 & $54 \%+46 \% 1 \mathrm{~s}^{2} 2 \mathrm{~s}^{1} 2 \mathrm{p}^{1}\left({ }^{3} \mathrm{P}\right) 4 \mathrm{f}^{14} \mathrm{G}$ \\
\hline 177 & $1 s^{2} 2 s^{1} 2 p^{1}\left({ }^{3} P\right) 4 f^{1}$ & ${ }^{4} \mathrm{~F}$ & 2.5 & 11644818 & $43 \%+30 \% 1 \mathrm{~s}^{2} 2 \mathrm{~s}^{1} 2 \mathrm{p}^{1}\left({ }^{3} \mathrm{P}\right) 4 \mathrm{f}^{14} \mathrm{D}+10 \% 1 \mathrm{~s}^{2} 2 \mathrm{~s}^{1} 2 \mathrm{p}^{1}\left({ }^{3} \mathrm{P}\right) 4 \mathrm{f}^{12} \mathrm{D}$ \\
\hline 178 & $1 s^{2} 2 s^{1} 2 p^{1}\left({ }^{3} P\right) 4 f^{1}$ & ${ }^{2} \mathrm{~F}$ & 3.5 & 11646915 & $46 \%+23 \% 1 s^{2} 2 s^{1} 2 p^{1}\left({ }^{3} P\right) 4 f^{14} D+18 \% 1 s^{2} 2 s^{1} 2 p^{1}\left({ }^{3} P\right) 4 f^{12} G$ \\
\hline 179 & $1 \mathrm{~s}^{2} 2 \mathrm{~s}^{1} 2 \mathrm{p}^{1}\left({ }^{3} \mathrm{P}\right) 4 \mathrm{f}^{1}$ & ${ }^{4} \mathrm{G}$ & 5.5 & 11646958 & $100 \%$ \\
\hline
\end{tabular}


Table 1. continued.

\begin{tabular}{|c|c|c|c|c|c|}
\hline Index & Configuration & $L S$ & $J$ & $E\left(\mathrm{~cm}^{-1}\right)$ & Composition \\
\hline 180 & $1 s^{2} 2 s^{1} 2 p^{1}\left({ }^{3} P\right) 4 d^{1}$ & ${ }^{2} \mathrm{P}$ & 0.5 & 11647548 & $90 \%$ \\
\hline 181 & $1 \mathrm{~s}^{2} 2 \mathrm{~s}^{1} 2 \mathrm{p}^{1}\left({ }^{3} \mathrm{P}\right) 4 \mathrm{f}^{1}$ & ${ }^{4} \mathrm{D}$ & 1.5 & 11648980 & $69 \%+23 \% 1 s^{2} 2 s^{1} 2 p^{1}\left({ }^{3} P\right) 4 f^{14} F$ \\
\hline 182 & $1 \mathrm{~s}^{2} 2 \mathrm{~s}^{1} 2 \mathrm{p}^{1}\left({ }^{3} \mathrm{P}\right) 4 \mathrm{f}^{1}$ & ${ }^{2} \mathrm{G}$ & 4.5 & 11650991 & $85 \%+10 \% 1 \mathrm{~s}^{2} 2 \mathrm{~s}^{1} 2 \mathrm{p}^{1}\left({ }^{3} \mathrm{P}\right) 4 \mathrm{f}^{14} \mathrm{G}$ \\
\hline 183 & $1 \mathrm{~s}^{2} 2 \mathrm{~s}^{1} 2 \mathrm{p}^{1}\left({ }^{3} \mathrm{P}\right) 4 \mathrm{f}^{1}$ & ${ }^{4} \mathrm{D}$ & 2.5 & 11651834 & $34 \%+33 \% 1 \mathrm{~s}^{2} 2 \mathrm{~s}^{1} 2 \mathrm{p}^{1}\left({ }^{3} \mathrm{P}\right) 4 \mathrm{f}^{1}{ }^{2} \mathrm{D}+31 \% 1 \mathrm{~s}^{2} 2 \mathrm{~s}^{1} 2 \mathrm{p}^{1}\left({ }^{3} \mathrm{P}\right) 4 \mathrm{f}^{1}{ }^{2} \mathrm{~F}$ \\
\hline 184 & $1 \mathrm{~s}^{2} 2 \mathrm{~s}^{1} 2 \mathrm{p}^{1}\left({ }^{3} \mathrm{P}\right) 4 \mathrm{f}^{1}$ & ${ }^{4} \mathrm{D}$ & 0.5 & 11652069 & $100 \%$ \\
\hline 185 & $1 \mathrm{~s}^{2} 2 \mathrm{~s}^{1} 2 \mathrm{p}^{1}\left({ }^{3} \mathrm{P}\right) 4 \mathrm{f}^{1}$ & ${ }^{2} \mathrm{D}$ & 1.5 & 11657791 & $79 \%+17 \% 1 \mathrm{~s}^{2} 2 \mathrm{~s}^{1} 2 \mathrm{p}^{1}\left({ }^{3} \mathrm{P}\right) 4 \mathrm{f}^{14} \mathrm{D}$ \\
\hline 186 & $1 s^{2} 2 s^{1} 2 p^{1}\left({ }^{1} \mathrm{P}\right) 4 s^{1}$ & ${ }^{2} \mathrm{P}$ & 0.5 & 11758032 & $96 \%$ \\
\hline 187 & $1 s^{2} 2 s^{1} 2 p^{1}\left({ }^{1} \mathrm{P}\right) 4 s^{1}$ & ${ }^{2} \mathrm{P}$ & 1.5 & 11759674 & $96 \%$ \\
\hline 188 & $1 s^{2} 2 s^{1} 2 p^{1}\left({ }^{1} P\right) 4 p^{1}$ & ${ }^{2} \mathrm{D}$ & 1.5 & 11832259 & $82 \%+14 \% 1 \mathrm{~s}^{2} 2 \mathrm{~s}^{1} 2 \mathrm{p}^{1}\left({ }^{1} \mathrm{P}\right) 4 \mathrm{p}^{12} \mathrm{P}$ \\
\hline 189 & $1 s^{2} 2 s^{1} 2 p^{1}\left({ }^{1} P\right) 4 p^{1}$ & ${ }^{2} \mathrm{P}$ & 0.5 & 11833806 & $85 \%+11 \% 1 \mathrm{~s}^{2} 2 \mathrm{~s}^{1} 2 \mathrm{p}^{1}\left({ }^{1} \mathrm{P}\right) 4 \mathrm{p}^{12} \mathrm{~S}$ \\
\hline 190 & $1 s^{2} 2 s^{1} 2 p^{1}\left({ }^{1} P\right) 4 p^{1}$ & ${ }^{2} \mathrm{D}$ & 2.5 & 11841574 & $96 \%$ \\
\hline 191 & $1 s^{2} 2 s^{1} 2 p^{1}\left({ }^{1} P\right) 4 p^{1}$ & ${ }^{2} \mathrm{P}$ & 1.5 & 11847478 & $82 \%+13 \% 1 s^{2} 2 s^{1} 2 p^{1}\left({ }^{1} P\right) 4 p^{1}{ }^{2} D$ \\
\hline 192 & $1 s^{2} 2 s^{1} 2 p^{1}\left({ }^{1} P\right) 4 p^{1}$ & ${ }^{2} \mathrm{~S}$ & 0.5 & 11855186 & $83 \%+11 \% 1 \mathrm{~s}^{2} 2 \mathrm{~s}^{1} 2 \mathrm{p}^{1}\left({ }^{1} \mathrm{P}\right) 4 \mathrm{p}^{1}{ }^{2} \mathrm{P}$ \\
\hline 193 & $1 s^{2} 2 s^{1} 2 p^{1}\left({ }^{1} P\right) 4 d^{1}$ & ${ }^{2} \mathrm{D}$ & 1.5 & 11902998 & $97 \%$ \\
\hline 194 & $1 \mathrm{~s}^{2} 2 \mathrm{~s}^{1} 2 \mathrm{p}^{1}\left({ }^{1} \mathrm{P}\right) 4 \mathrm{~d}^{1}$ & ${ }^{2} \mathrm{~F}$ & 2.5 & 11903215 & $91 \%$ \\
\hline 195 & $1 \mathrm{~s}^{2} 2 \mathrm{~s}^{1} 2 \mathrm{p}^{1}\left({ }^{1} \mathrm{P}\right) 4 \mathrm{~d}^{1}$ & ${ }^{2} \mathrm{~F}$ & 3.5 & 11903365 & $97 \%$ \\
\hline 196 & $1 \mathrm{~s}^{2} 2 \mathrm{~s}^{1} 2 \mathrm{p}^{1}\left({ }^{1} \mathrm{P}\right) 4 \mathrm{~d}^{1}$ & ${ }^{2} \mathrm{D}$ & 2.5 & 11908277 & $91 \%$ \\
\hline 197 & $1 \mathrm{~s}^{2} 2 \mathrm{~s}^{1} 2 \mathrm{p}^{1}\left({ }^{1} \mathrm{P}\right) 4 \mathrm{~d}^{1}$ & ${ }^{2} \mathrm{P}$ & 0.5 & 11916727 & $97 \%$ \\
\hline 198 & $1 \mathrm{~s}^{2} 2 \mathrm{~s}^{1} 2 \mathrm{p}^{1}\left({ }^{1} \mathrm{P}\right) 4 \mathrm{~d}^{1}$ & ${ }^{2} \mathrm{P}$ & 1.5 & 11920666 & $96 \%$ \\
\hline 199 & $1 \mathrm{~s}^{2} 2 \mathrm{~s}^{1} 2 \mathrm{p}^{1}\left({ }^{1} \mathrm{P}\right) 4 \mathrm{f}^{1}$ & ${ }^{2} \mathrm{~F}$ & 2.5 & 11932709 & $97 \%$ \\
\hline 200 & $1 \mathrm{~s}^{2} 2 \mathrm{~s}^{1} 2 \mathrm{p}^{1}\left({ }^{1} \mathrm{P}\right) 4 \mathrm{f}^{1}$ & ${ }^{2} \mathrm{~F}$ & 3.5 & 11934584 & $97 \%$ \\
\hline 201 & $1 \mathrm{~s}^{2} 2 \mathrm{~s}^{1} 2 \mathrm{p}^{1}\left({ }^{1} \mathrm{P}\right) 4 \mathrm{f}^{1}$ & ${ }^{2} \mathrm{G}$ & 3.5 & 11941218 & $97 \%$ \\
\hline 202 & $1 \mathrm{~s}^{2} 2 \mathrm{~s}^{1} 2 \mathrm{p}^{1}\left({ }^{1} \mathrm{P}\right) 4 \mathrm{f}^{1}$ & ${ }^{2} \mathrm{G}$ & 4.5 & 11942845 & $97 \%$ \\
\hline 203 & $1 \mathrm{~s}^{2} 2 \mathrm{~s}^{1} 2 \mathrm{p}^{1}\left({ }^{1} \mathrm{P}\right) 4 \mathrm{f}^{1}$ & ${ }^{2} \mathrm{D}$ & 1.5 & 11948864 & $97 \%$ \\
\hline 204 & $1 \mathrm{~s}^{2} 2 \mathrm{~s}^{1} 2 \mathrm{p}^{1}\left({ }^{1} \mathrm{P}\right) 4 \mathrm{f}^{1}$ & ${ }^{2} \mathrm{D}$ & 2.5 & 11950454 & $97 \%$ \\
\hline 205 & $1 \mathrm{~s}^{2} 2 \mathrm{p}^{2}\left({ }^{3} \mathrm{P}\right) 4 \mathrm{~s}^{1}$ & ${ }^{4} \mathrm{P}$ & 0.5 & 11966282 & $72 \%+20 \% 1 \mathrm{~s}^{2} 2 \mathrm{p}^{2}\left({ }^{3} \mathrm{P}\right) 4 \mathrm{~s}^{1}{ }^{2} \mathrm{P}$ \\
\hline 206 & $1 \mathrm{~s}^{2} 2 \mathrm{p}^{2}\left({ }^{3} \mathrm{P}\right) 4 \mathrm{p}^{1}$ & ${ }^{4} \mathrm{D}$ & 0.5 & 12026218 & $57 \%+13 \% 1 \mathrm{~s}^{2} 2 \mathrm{p}^{2}\left({ }^{3} \mathrm{P}\right) 4 \mathrm{p}^{12} \mathrm{~S}+13 \% 1 \mathrm{~s}^{2} 2 \mathrm{p}^{2}\left({ }^{3} \mathrm{P}\right) 4 \mathrm{p}^{12} \mathrm{P}$ \\
\hline 207 & $1 \mathrm{~s}^{2} 2 \mathrm{p}^{2}\left({ }^{3} \mathrm{P}\right) 4 \mathrm{~s}^{1}$ & ${ }^{4} \mathrm{P}$ & 1.5 & 12032154 & $90 \%$ \\
\hline 208 & $1 \mathrm{~s}^{2} 2 \mathrm{p}^{2}\left({ }^{3} \mathrm{P}\right) 4 \mathrm{~s}^{1}$ & ${ }^{2} \mathrm{P}$ & 0.5 & 12044766 & $75 \%+23 \% 1 \mathrm{~s}^{2} 2 \mathrm{p}^{2}\left({ }^{3} \mathrm{P}\right) 4 \mathrm{~s}^{1}{ }^{4} \mathrm{P}$ \\
\hline 209 & $1 \mathrm{~s}^{2} 2 \mathrm{p}^{2}\left({ }^{3} \mathrm{P}\right) 4 \mathrm{p}^{1}$ & ${ }^{4} \mathrm{D}$ & 1.5 & 12048491 & $41 \%+28 \% 1 \mathrm{~s}^{2} 2 \mathrm{p}^{2}\left({ }^{3} \mathrm{P}\right) 4 \mathrm{p}^{1}{ }^{4} \mathrm{P}+16 \% 1 \mathrm{~s}^{2} 2 \mathrm{p}^{2}\left({ }^{3} \mathrm{P}\right) 4 \mathrm{p}^{1}{ }^{2} \mathrm{D}$ \\
\hline 210 & $1 \mathrm{~s}^{2} 2 \mathrm{p}^{2}\left({ }^{3} \mathrm{P}\right) 4 \mathrm{~s}^{1}$ & ${ }^{4} \mathrm{P}$ & 2.5 & 12077037 & $74 \%+22 \% 1 \mathrm{~s}^{2} 2 \mathrm{p}^{2}\left({ }^{1} \mathrm{D}\right) 4 \mathrm{~s}^{12} \mathrm{D}$ \\
\hline 211 & $1 s^{2} 2 p^{2}\left({ }^{3} \mathrm{P}\right) 4 p^{1}$ & ${ }^{2} \mathrm{~S}$ & 0.5 & 12090712 & $36 \%+32 \% 1 \mathrm{~s}^{2} 2 \mathrm{p}^{2}\left({ }^{3} \mathrm{P}\right) 4 \mathrm{p}^{14} \mathrm{D}+32 \% 1 \mathrm{~s}^{2} 2 \mathrm{p}^{2}\left({ }^{3} \mathrm{P}\right) 4 \mathrm{p}^{14} \mathrm{P}$ \\
\hline 212 & $1 \mathrm{~s}^{2} 2 \mathrm{p}^{2}\left({ }^{3} \mathrm{P}\right) 4 \mathrm{~d}^{1}$ & ${ }^{4} \mathrm{~F}$ & 1.5 & 12091037 & $31 \%+27 \% 1 \mathrm{~s}^{2} 2 \mathrm{p}^{2}\left({ }^{3} \mathrm{P}\right) 4 \mathrm{~s}^{1}{ }^{2} \mathrm{P}+12 \% 1 \mathrm{~s}^{2} 2 \mathrm{p}^{2}\left({ }^{1} \mathrm{D}\right) 4 \mathrm{~s}^{1}{ }^{2} \mathrm{D}$ \\
\hline 213 & $1 \mathrm{~s}^{2} 2 \mathrm{p}^{2}\left({ }^{3} \mathrm{P}\right) 4 \mathrm{~s}^{1}$ & ${ }^{2} \mathrm{P}$ & 1.5 & 12096944 & $38 \%+24 \% 1 \mathrm{~s}^{2} 2 \mathrm{p}^{2}\left({ }^{3} \mathrm{P}\right) 4 \mathrm{~d}^{14} \mathrm{~F}+16 \% 1 \mathrm{~s}^{2} 2 \mathrm{p}^{2}\left({ }^{1} \mathrm{D}\right) 4 \mathrm{~s}^{1}{ }^{2} \mathrm{D}$ \\
\hline 214 & $1 \mathrm{~s}^{2} 2 \mathrm{p}^{2}\left({ }^{3} \mathrm{P}\right) 4 \mathrm{p}^{1}$ & ${ }^{4} \mathrm{D}$ & 1.5 & 12104155 & $51 \%+14 \% 1 \mathrm{~s}^{2} 2 \mathrm{p}^{2}\left({ }^{3} \mathrm{P}\right) 4 \mathrm{p}^{12} \mathrm{D}+12 \% 1 \mathrm{~s}^{2} 2 \mathrm{p}^{2}\left({ }^{3} \mathrm{P}\right) 4 \mathrm{p}^{1}{ }^{2} \mathrm{P}$ \\
\hline 215 & $1 \mathrm{~s}^{2} 2 \mathrm{p}^{2}\left({ }^{3} \mathrm{P}\right) 4 \mathrm{~d}^{1}$ & ${ }^{4} \mathrm{~F}$ & 2.5 & 12109479 & $33 \%+28 \% 1 \mathrm{~s}^{2} 2 \mathrm{p}^{2}\left({ }^{3} \mathrm{P}\right) 4 \mathrm{~d}^{14} \mathrm{D}+15 \% 1 \mathrm{~s}^{2} 2 \mathrm{p}^{2}\left({ }^{3} \mathrm{P}\right) 4 \mathrm{~d}^{12} \mathrm{~F}$ \\
\hline 216 & $1 s^{2} 2 p^{2}\left({ }^{3} P\right) 4 p^{1}$ & ${ }^{4} \mathrm{D}$ & 2.5 & 12109603 & $68 \%+24 \% 1 \mathrm{~s}^{2} 2 \mathrm{p}^{2}\left({ }^{3} \mathrm{P}\right) 4 \mathrm{p}^{14} \mathrm{P}$ \\
\hline 217 & $1 \mathrm{~s}^{2} 2 \mathrm{p}^{2}\left({ }^{3} \mathrm{P}\right) 4 \mathrm{p}^{1}$ & ${ }^{4} \mathrm{P}$ & 0.5 & 12119410 & $50 \%+23 \% 1 \mathrm{~s}^{2} 2 \mathrm{p}^{2}\left({ }^{3} \mathrm{P}\right) 4 \mathrm{p}^{1}{ }^{2} \mathrm{P}+20 \% 1 \mathrm{~s}^{2} 2 \mathrm{p}^{2}\left({ }^{3} \mathrm{P}\right) 4 \mathrm{p}^{1}{ }^{2} \mathrm{~S}$ \\
\hline 218 & $1 s^{2} 2 p^{2}\left({ }^{3} P\right) 4 p^{1}$ & ${ }^{2} \mathrm{D}$ & 1.5 & 12125947 & $58 \%+23 \% 1 \mathrm{~s}^{2} 2 \mathrm{p}^{2}\left({ }^{3} \mathrm{P}\right) 4 \mathrm{p}^{14} \mathrm{P}+15 \% 1 \mathrm{~s}^{2} 2 \mathrm{p}^{2}\left({ }^{3} \mathrm{P}\right) 4 \mathrm{p}^{14} \mathrm{~S}$ \\
\hline 219 & $1 \mathrm{~s}^{2} 2 \mathrm{p}^{2}\left({ }^{3} \mathrm{P}\right) 4 \mathrm{f}^{1}$ & ${ }^{4} \mathrm{G}$ & 2.5 & 12133488 & $43 \%+17 \% 1 \mathrm{~s}^{2} 2 \mathrm{p}^{2}\left({ }^{3} \mathrm{P}\right) 4 \mathrm{f}^{1}{ }^{2} \mathrm{D}+15 \% 1 \mathrm{~s}^{2} 2 \mathrm{p}^{2}\left({ }^{3} \mathrm{P}\right) 4 \mathrm{f}^{14} \mathrm{~F}$ \\
\hline 220 & $1 \mathrm{~s}^{2} 2 \mathrm{p}^{2}\left({ }^{3} \mathrm{P}\right) 4 \mathrm{f}^{1}$ & ${ }^{4} \mathrm{D}$ & 3.5 & 12136390 & $24 \%+23 \% 1 \mathrm{~s}^{2} 2 \mathrm{p}^{2}\left({ }^{3} \mathrm{P}\right) 4 \mathrm{f}^{12} \mathrm{G}+21 \% 1 \mathrm{~s}^{2} 2 \mathrm{p}^{2}\left({ }^{3} \mathrm{P}\right) 4 \mathrm{f}^{14} \mathrm{~F}$ \\
\hline 221 & $1 s^{2} 2 p^{2}\left({ }^{3} P\right) 4 p^{1}$ & ${ }^{4} \mathrm{P}$ & 2.5 & 12142023 & $31 \%+22 \% 1 \mathrm{~s}^{2} 2 \mathrm{p}^{2}\left({ }^{3} \mathrm{P}\right) 4 \mathrm{p}^{1}{ }^{4} \mathrm{D}+19 \% 1 \mathrm{~s}^{2} 2 \mathrm{p}^{2}\left({ }^{3} \mathrm{P}\right) 4 \mathrm{p}^{12} \mathrm{D}$ \\
\hline 222 & $1 \mathrm{~s}^{2} 2 \mathrm{p}^{2}\left({ }^{3} \mathrm{P}\right) 4 \mathrm{p}^{1}$ & ${ }^{4} \mathrm{D}$ & 3.5 & 12153226 & $77 \%+23 \% 1 \mathrm{~s}^{2} 2 \mathrm{p}^{2}\left({ }^{1} \mathrm{D}\right) 4 \mathrm{p}^{12} \mathrm{~F}$ \\
\hline 223 & $1 s^{2} 2 p^{2}\left({ }^{3} P\right) 4 p^{1}$ & ${ }^{4} \mathrm{~S}$ & 1.5 & 12163250 & $50 \%+26 \% 1 \mathrm{~s}^{2} 2 \mathrm{p}^{2}\left({ }^{3} \mathrm{P}\right) 4 \mathrm{p}^{14} \mathrm{P}+16 \% 1 \mathrm{~s}^{2} 2 \mathrm{p}^{2}\left({ }^{1} \mathrm{D}\right) 4 \mathrm{p}^{12} \mathrm{P}$ \\
\hline 224 & $1 \mathrm{~s}^{2} 2 \mathrm{p}^{2}\left({ }^{3} \mathrm{P}\right) 4 \mathrm{~d}^{1}$ & ${ }^{4} \mathrm{~F}$ & 1.5 & 12165218 & $36 \%+35 \% 1 \mathrm{~s}^{2} 2 \mathrm{p}^{2}\left({ }^{3} \mathrm{P}\right) 4 \mathrm{~d}^{1}{ }^{2} \mathrm{P}+18 \% 1 \mathrm{~s}^{2} 2 \mathrm{p}^{2}\left({ }^{3} \mathrm{P}\right) 4 \mathrm{~d}^{14} \mathrm{D}$ \\
\hline 225 & $1 \mathrm{~s}^{2} 2 \mathrm{p}^{2}\left({ }^{3} \mathrm{P}\right) 4 \mathrm{~d}^{1}$ & ${ }^{4} \mathrm{~F}$ & 3.5 & 12167484 & $59 \%+33 \% 1 \mathrm{~s}^{2} 2 \mathrm{p}^{2}\left({ }^{3} \mathrm{P}\right) 4 \mathrm{~d}^{14} \mathrm{D}$ \\
\hline 226 & $1 \mathrm{~s}^{2} 2 \mathrm{p}^{2}\left({ }^{3} \mathrm{P}\right) 4 \mathrm{~d}^{1}$ & ${ }^{4} \mathrm{D}$ & 0.5 & 12168370 & $80 \%+14 \% 1 \mathrm{~s}^{2} 2 \mathrm{p}^{2}\left({ }^{3} \mathrm{P}\right) 4 \mathrm{~d}^{1}{ }^{2} \mathrm{P}$ \\
\hline 227 & $1 s^{2} 2 p^{2}\left({ }^{3} P\right) 4 p^{1}$ & ${ }^{2} \mathrm{P}$ & 1.5 & 12170620 & $58 \%+22 \% 1 \mathrm{~s}^{2} 2 \mathrm{p}^{2}\left({ }^{1} \mathrm{D}\right) 4 \mathrm{p}^{12} \mathrm{D}$ \\
\hline 228 & $1 \mathrm{~s}^{2} 2 \mathrm{p}^{2}\left({ }^{3} \mathrm{P}\right) 4 \mathrm{~d}^{1}$ & ${ }^{4} \mathrm{~F}$ & 2.5 & 12172277 & $56 \%+23 \% 1 \mathrm{~s}^{2} 2 \mathrm{p}^{2}\left({ }^{3} \mathrm{P}\right) 4 \mathrm{~d}^{14} \mathrm{P}$ \\
\hline 229 & $1 \mathrm{~s}^{2} 2 \mathrm{p}^{2}\left({ }^{3} \mathrm{P}\right) 4 \mathrm{p}^{1}$ & ${ }^{2} \mathrm{D}$ & 2.5 & 12179277 & $45 \%+27 \% 1 \mathrm{~s}^{2} 2 \mathrm{p}^{2}\left({ }^{3} \mathrm{P}\right) 4 \mathrm{p}^{1}{ }^{4} \mathrm{P}+18 \% 1 \mathrm{~s}^{2} 2 \mathrm{p}^{2}\left({ }^{1} \mathrm{D}\right) 4 \mathrm{p}^{12} \mathrm{~F}$ \\
\hline 230 & $1 \mathrm{~s}^{2} 2 \mathrm{p}^{2}\left({ }^{3} \mathrm{P}\right) 4 \mathrm{p}^{1}$ & ${ }^{2} \mathrm{P}$ & 0.5 & 12185339 & $57 \%+22 \% 1 \mathrm{~s}^{2} 2 \mathrm{p}^{2}\left({ }^{3} \mathrm{P}\right) 4 \mathrm{p}^{1}{ }^{2} \mathrm{~S}+11 \% 1 \mathrm{~s}^{2} 2 \mathrm{p}^{2}\left({ }^{1} \mathrm{D}\right) 4 \mathrm{p}^{12} \mathrm{P}$ \\
\hline 231 & $1 \mathrm{~s}^{2} 2 \mathrm{p}^{2}\left({ }^{3} \mathrm{P}\right) 4 \mathrm{~d}^{1}$ & ${ }^{4} \mathrm{D}$ & 1.5 & 12186252 & $37 \%+28 \% 1 \mathrm{~s}^{2} 2 \mathrm{p}^{2}\left({ }^{3} \mathrm{P}\right) 4 \mathrm{~d}^{1}{ }^{2} \mathrm{P}+17 \% 1 \mathrm{~s}^{2} 2 \mathrm{p}^{2}\left({ }^{3} \mathrm{P}\right) 4 \mathrm{~d}^{12} \mathrm{D}$ \\
\hline 232 & $1 \mathrm{~s}^{2} 2 \mathrm{p}^{2}\left({ }^{3} \mathrm{P}\right) 4 \mathrm{~d}^{1}$ & ${ }^{2} \mathrm{~F}$ & 2.5 & 12187456 & $64 \%+21 \% 1 \mathrm{~s}^{2} 2 \mathrm{p}^{2}\left({ }^{3} \mathrm{P}\right) 4 \mathrm{~d}^{14} \mathrm{P}$ \\
\hline 233 & $1 \mathrm{~s}^{2} 2 \mathrm{p}^{2}\left({ }^{3} \mathrm{P}\right) 4 \mathrm{f}^{1}$ & ${ }^{4} \mathrm{G}$ & 2.5 & 12200490 & $44 \%+35 \% 1 \mathrm{~s}^{2} 2 \mathrm{p}^{2}\left({ }^{3} \mathrm{P}\right) 4 \mathrm{f}^{12} \mathrm{D}+15 \% 1 \mathrm{~s}^{2} 2 \mathrm{p}^{2}\left({ }^{3} \mathrm{P}\right) 4 \mathrm{f}^{14} \mathrm{D}$ \\
\hline 234 & $1 \mathrm{~s}^{2} 2 \mathrm{p}^{2}\left({ }^{3} \mathrm{P}\right) 4 \mathrm{f}^{1}$ & ${ }^{4} \mathrm{D}$ & 3.5 & 12202351 & $44 \%+42 \% 1 \mathrm{~s}^{2} 2 \mathrm{p}^{2}\left({ }^{3} \mathrm{P}\right) 4 \mathrm{f}^{1}{ }^{4} \mathrm{G}$ \\
\hline 235 & $1 \mathrm{~s}^{2} 2 \mathrm{p}^{2}\left({ }^{3} \mathrm{P}\right) 4 \mathrm{f}^{1}$ & ${ }^{4} \mathrm{G}$ & 4.5 & 12205379 & $53 \%+31 \% 1 \mathrm{~s}^{2} 2 \mathrm{p}^{2}\left({ }^{3} \mathrm{P}\right) 4 \mathrm{f}^{1}{ }^{4} \mathrm{~F}+16 \% 1 \mathrm{~s}^{2} 2 \mathrm{p}^{2}\left({ }^{3} \mathrm{P}\right) 4 \mathrm{f}^{12} \mathrm{G}$ \\
\hline 236 & $1 \mathrm{~s}^{2} 2 \mathrm{p}^{2}\left({ }^{3} \mathrm{P}\right) 4 \mathrm{f}^{1}$ & ${ }^{2} \mathrm{G}$ & 3.5 & 12205809 & $52 \%+18 \% 1 \mathrm{~s}^{2} 2 \mathrm{p}^{2}\left({ }^{3} \mathrm{P}\right) 4 \mathrm{f}^{14} \mathrm{G}+15 \% 1 \mathrm{~s}^{2} 2 \mathrm{p}^{2}\left({ }^{3} \mathrm{P}\right) 4 \mathrm{f}^{12} \mathrm{~F}$ \\
\hline 237 & $1 \mathrm{~s}^{2} 2 \mathrm{p}^{2}\left({ }^{3} \mathrm{P}\right) 4 \mathrm{f}^{1}$ & ${ }^{4} \mathrm{~F}$ & 1.5 & 12206952 & $59 \%+21 \% 1 \mathrm{~s}^{2} 2 \mathrm{p}^{2}\left({ }^{3} \mathrm{P}\right) 4 \mathrm{f}^{1}{ }^{4} \mathrm{D}+20 \% 1 \mathrm{~s}^{2} 2 \mathrm{p}^{2}\left({ }^{3} \mathrm{P}\right) 4 \mathrm{f}^{12} \mathrm{D}$ \\
\hline 238 & $1 \mathrm{~s}^{2} 2 \mathrm{p}^{2}\left({ }^{3} \mathrm{P}\right) 4 \mathrm{~d}^{1}$ & ${ }^{4} \mathrm{D}$ & 3.5 & 12207587 & $32 \%+29 \% 1 \mathrm{~s}^{2} 2 \mathrm{p}^{2}\left({ }^{3} \mathrm{P}\right) 4 \mathrm{~d}^{14} \mathrm{~F}+17 \% 1 \mathrm{~s}^{2} 2 \mathrm{p}^{2}\left({ }^{1} \mathrm{D}\right) 4 \mathrm{~d}^{1}{ }^{2} \mathrm{~F}$ \\
\hline
\end{tabular}


Table 1. continued.

\begin{tabular}{|c|c|c|c|c|c|}
\hline Index & Configuration & $L S$ & $J$ & $E\left(\mathrm{~cm}^{-1}\right)$ & Composition \\
\hline 239 & $1 \mathrm{~s}^{2} 2 \mathrm{p}^{2}\left({ }^{3} \mathrm{P}\right) 4 \mathrm{~d}^{1}$ & ${ }^{4} \mathrm{~F}$ & 4.5 & 12209498 & $78 \%+22 \% 1 \mathrm{~s}^{2} 2 \mathrm{p}^{2}\left({ }^{1} \mathrm{D}\right) 4 \mathrm{~d}^{12} \mathrm{G}$ \\
\hline 240 & $1 \mathrm{~s}^{2} 2 \mathrm{p}^{2}\left({ }^{3} \mathrm{P}\right) 4 \mathrm{f}^{1}$ & ${ }^{2} \mathrm{~F}$ & 2.5 & 12210388 & $30 \%+29 \% 1 \mathrm{~s}^{2} 2 \mathrm{p}^{2}\left({ }^{3} \mathrm{P}\right) 4 \mathrm{f}^{1}{ }^{4} \mathrm{D}+24 \% 1 \mathrm{~s}^{2} 2 \mathrm{p}^{2}\left({ }^{3} \mathrm{P}\right) 4 \mathrm{f}^{1}{ }^{4} \mathrm{~F}$ \\
\hline 241 & $1 \mathrm{~s}^{2} 2 \mathrm{p}^{2}\left({ }^{1} \mathrm{D}\right) 4 \mathrm{~s}^{1}$ & ${ }^{2} \mathrm{D}$ & 2.5 & 12213901 & $56 \%+17 \% 1 \mathrm{~s}^{2} 2 \mathrm{p}^{2}\left({ }^{3} \mathrm{P}\right) 4 \mathrm{~s}^{14} \mathrm{P}$ \\
\hline 242 & $1 \mathrm{~s}^{2} 2 \mathrm{p}^{2}\left({ }^{1} \mathrm{D}\right) 4 \mathrm{~s}^{1}$ & ${ }^{2} \mathrm{D}$ & 1.5 & 12221564 & $68 \%+24 \% 1 \mathrm{~s}^{2} 2 \mathrm{p}^{2}\left({ }^{3} \mathrm{P}\right) 4 \mathrm{~s}^{12} \mathrm{P}$ \\
\hline 243 & $1 \mathrm{~s}^{2} 2 \mathrm{p}^{2}\left({ }^{3} \mathrm{P}\right) 4 \mathrm{~d}^{1}$ & ${ }^{4} \mathrm{D}$ & 2.5 & 12222169 & $37 \%+18 \% 1 \mathrm{~s}^{2} 2 \mathrm{p}^{2}\left({ }^{3} \mathrm{P}\right) 4 \mathrm{~d}^{14} \mathrm{P}$ \\
\hline 244 & $1 \mathrm{~s}^{2} 2 \mathrm{p}^{2}\left({ }^{3} \mathrm{P}\right) 4 \mathrm{~d}^{1}$ & ${ }^{4} \mathrm{P}$ & 1.5 & 12227306 & $57 \%+19 \% 1 \mathrm{~s}^{2} 2 \mathrm{p}^{2}\left({ }^{3} \mathrm{P}\right) 4 \mathrm{~d}^{14} \mathrm{D}+13 \% 1 \mathrm{~s}^{2} 2 \mathrm{p}^{2}\left({ }^{1} \mathrm{D}\right) 4 \mathrm{~d}^{12} \mathrm{P}$ \\
\hline 245 & $1 \mathrm{~s}^{2} 2 \mathrm{p}^{2}\left({ }^{3} \mathrm{P}\right) 4 \mathrm{~d}^{1}$ & ${ }^{4} \mathrm{P}$ & 0.5 & 12230405 & $72 \%+17 \% 1 \mathrm{~s}^{2} 2 \mathrm{p}^{2}\left({ }^{1} \mathrm{D}\right) 4 \mathrm{~d}^{12} \mathrm{~S}$ \\
\hline 246 & $1 \mathrm{~s}^{2} 2 \mathrm{p}^{2}\left({ }^{3} \mathrm{P}\right) 4 \mathrm{~d}^{1}$ & ${ }^{2} \mathrm{P}$ & 0.5 & 12236303 & $68 \%+14 \% 1 \mathrm{~s}^{2} 2 \mathrm{p}^{2}\left({ }^{3} \mathrm{P}\right) 4 \mathrm{~d}^{14} \mathrm{D}+11 \% 1 \mathrm{~s}^{2} 2 \mathrm{p}^{2}\left({ }^{1} \mathrm{D}\right) 4 \mathrm{~d}^{12} \mathrm{P}$ \\
\hline 247 & $1 \mathrm{~s}^{2} 2 \mathrm{p}^{2}\left({ }^{3} \mathrm{P}\right) 4 \mathrm{~d}^{1}$ & ${ }^{2} \mathrm{~F}$ & 3.5 & 12243343 & $54 \%+21 \% 1 \mathrm{~s}^{2} 2 \mathrm{p}^{2}\left({ }^{1} \mathrm{D}\right) 4 \mathrm{~d}^{12} \mathrm{G}+18 \% 1 \mathrm{~s}^{2} 2 \mathrm{p}^{2}\left({ }^{3} \mathrm{P}\right) 4 \mathrm{~d}^{14} \mathrm{D}$ \\
\hline 248 & $1 \mathrm{~s}^{2} 2 \mathrm{p}^{2}\left({ }^{3} \mathrm{P}\right) 4 \mathrm{~d}^{1}$ & ${ }^{2} \mathrm{D}$ & 1.5 & 12244386 & $58 \%+15 \% 1 \mathrm{~s}^{2} 2 \mathrm{p}^{2}\left({ }^{1} \mathrm{D}\right) 4 \mathrm{~d}^{12} \mathrm{D}+10 \% 1 \mathrm{~s}^{2} 2 \mathrm{p}^{2}\left({ }^{3} \mathrm{P}\right) 4 \mathrm{~d}^{1}{ }^{2} \mathrm{P}$ \\
\hline 249 & $1 \mathrm{~s}^{2} 2 \mathrm{p}^{2}\left({ }^{3} \mathrm{P}\right) 4 \mathrm{~d}^{1}$ & ${ }^{2} \mathrm{D}$ & 2.5 & 12244441 & $53 \%+19 \% 1 \mathrm{~s}^{2} 2 \mathrm{p}^{2}\left({ }^{1} \mathrm{D}\right) 4 \mathrm{~d}^{12} \mathrm{~F}$ \\
\hline 250 & $1 \mathrm{~s}^{2} 2 \mathrm{p}^{2}\left({ }^{3} \mathrm{P}\right) 4 \mathrm{f}^{1}$ & ${ }^{4} \mathrm{G}$ & 4.5 & 12248830 & $32 \%+31 \% 1 \mathrm{~s}^{2} 2 \mathrm{p}^{2}\left({ }^{3} \mathrm{P}\right) 4 \mathrm{f}^{1}{ }^{2} \mathrm{G}+18 \% 1 \mathrm{~s}^{2} 2 \mathrm{p}^{2}\left({ }^{1} \mathrm{D}\right) 4 \mathrm{f}^{12} \mathrm{H}$ \\
\hline 251 & $1 \mathrm{~s}^{2} 2 \mathrm{p}^{2}\left({ }^{3} \mathrm{P}\right) 4 \mathrm{f}^{1}$ & ${ }^{4} \mathrm{G}$ & 5.5 & 12249213 & $77 \%+23 \% 1 \mathrm{~s}^{2} 2 \mathrm{p}^{2}\left({ }^{1} \mathrm{D}\right) 4 \mathrm{f}^{12} \mathrm{H}$ \\
\hline 252 & $1 \mathrm{~s}^{2} 2 \mathrm{p}^{2}\left({ }^{3} \mathrm{P}\right) 4 \mathrm{f}^{1}$ & ${ }^{4} \mathrm{D}$ & 0.5 & 12251273 & $79 \%+20 \% 1 \mathrm{~s}^{2} 2 \mathrm{p}^{2}\left({ }^{1} \mathrm{D}\right) 4 \mathrm{f}^{1}{ }^{2} \mathrm{P}$ \\
\hline 253 & $1 \mathrm{~s}^{2} 2 \mathrm{p}^{2}\left({ }^{3} \mathrm{P}\right) 4 \mathrm{f}^{1}$ & ${ }^{4} \mathrm{~F}$ & 3.5 & 12251580 & $42 \%+19 \% 1 \mathrm{~s}^{2} 2 \mathrm{p}^{2}\left({ }^{1} \mathrm{D}\right) 4 \mathrm{f}^{12} \mathrm{G}+13 \% 1 \mathrm{~s}^{2} 2 \mathrm{p}^{2}\left({ }^{3} \mathrm{P}\right) 4 \mathrm{f}^{1{ }^{4} \mathrm{G}}$ \\
\hline 254 & $1 \mathrm{~s}^{2} 2 \mathrm{p}^{2}\left({ }^{3} \mathrm{P}\right) 4 \mathrm{f}^{1}$ & ${ }^{4} \mathrm{D}$ & 1.5 & 12251859 & $61 \%+15 \% 1 \mathrm{~s}^{2} 2 \mathrm{p}^{2}\left({ }^{3} \mathrm{P}\right) 4 \mathrm{f}^{1}{ }^{4} \mathrm{~F}+11 \% 1 \mathrm{~s}^{2} 2 \mathrm{p}^{2}\left({ }^{1} \mathrm{D}\right) 4 \mathrm{f}^{1}{ }^{2} \mathrm{P}$ \\
\hline 255 & $1 \mathrm{~s}^{2} 2 \mathrm{p}^{2}\left({ }^{3} \mathrm{P}\right) 4 \mathrm{f}^{1}$ & ${ }^{4} \mathrm{~F}$ & 2.5 & 12252205 & $42 \%+26 \% 1 \mathrm{~s}^{2} 2 \mathrm{p}^{2}\left({ }^{3} \mathrm{P}\right) 4 \mathrm{f}^{1}{ }^{4} \mathrm{D}+18 \% 1 \mathrm{~s}^{2} 2 \mathrm{p}^{2}\left({ }^{1} \mathrm{D}\right) 4 \mathrm{f}^{12} \mathrm{~F}$ \\
\hline 256 & $1 \mathrm{~s}^{2} 2 \mathrm{p}^{2}\left({ }^{3} \mathrm{P}\right) 4 \mathrm{f}^{1}$ & ${ }^{4} \mathrm{~F}$ & 4.5 & 12254349 & $38 \%+32 \% 1 \mathrm{~s}^{2} 2 \mathrm{p}^{2}\left({ }^{3} \mathrm{P}\right) 4 \mathrm{f}^{12} \mathrm{G}+21 \% 1 \mathrm{~s}^{2} 2 \mathrm{p}^{2}\left({ }^{1} \mathrm{D}\right) 4 \mathrm{f}^{12} \mathrm{G}$ \\
\hline 257 & $1 \mathrm{~s}^{2} 2 \mathrm{p}^{2}\left({ }^{3} \mathrm{P}\right) 4 \mathrm{f}^{1}$ & ${ }^{2} \mathrm{D}$ & 1.5 & 12254654 & $60 \%+17 \% 1 \mathrm{~s}^{2} 2 \mathrm{p}^{2}\left({ }^{3} \mathrm{P}\right) 4 \mathrm{f}^{1}{ }^{4} \mathrm{~F}+12 \% 1 \mathrm{~s}^{2} 2 \mathrm{p}^{2}\left({ }^{1} \mathrm{D}\right) 4 \mathrm{f}^{1}{ }^{2} \mathrm{D}$ \\
\hline 258 & $1 \mathrm{~s}^{2} 2 \mathrm{p}^{2}\left({ }^{3} \mathrm{P}\right) 4 \mathrm{f}^{1}$ & ${ }^{2} \mathrm{~F}$ & 3.5 & 12255378 & $43 \%+19 \% 1 \mathrm{~s}^{2} 2 \mathrm{p}^{2}\left({ }^{1} \mathrm{D}\right) 4 \mathrm{f}^{12} \mathrm{~F}+11 \% 1 \mathrm{~s}^{2} 2 \mathrm{p}^{2}\left({ }^{3} \mathrm{P}\right) 4 \mathrm{f}^{14} \mathrm{D}$ \\
\hline 259 & $1 s^{2} 2 p^{2}\left({ }^{3} \mathrm{P}\right) 4 \mathrm{f}^{1}$ & ${ }^{2} \mathrm{~F}$ & 2.5 & 12255749 & $42 \%+19 \% 1 \mathrm{~s}^{2} 2 \mathrm{p}^{2}\left({ }^{3} \mathrm{P}\right) 4 \mathrm{f}^{12} \mathrm{D}+16 \% 1 \mathrm{~s}^{2} 2 \mathrm{p}^{2}\left({ }^{1} \mathrm{D}\right) 4 \mathrm{f}^{12} \mathrm{D}$ \\
\hline 260 & $1 s^{2} 2 s^{2} 5 s^{1}$ & ${ }^{2} \mathrm{~S}$ & 0.5 & 12279291 & $95 \%$ \\
\hline 261 & $1 s^{2} 2 p^{2}\left({ }^{1} D\right) 4 p^{1}$ & ${ }^{2} \mathrm{~F}$ & 2.5 & 12289009 & $53 \%+20 \% 1 \mathrm{~s}^{2} 2 \mathrm{p}^{2}\left({ }^{1} \mathrm{D}\right) 4 \mathrm{p}^{12} \mathrm{D}+19 \% 1 \mathrm{~s}^{2} 2 \mathrm{p}^{2}\left({ }^{3} \mathrm{P}\right) 4 \mathrm{p}^{12} \mathrm{D}$ \\
\hline 262 & $1 s^{2} 2 p^{2}\left({ }^{1} \mathrm{D}\right) 4 p^{1}$ & ${ }^{2} \mathrm{D}$ & 1.5 & 12293068 & $45 \%+30 \% 1 \mathrm{~s}^{2} 2 \mathrm{p}^{2}\left({ }^{1} \mathrm{D}\right) 4 \mathrm{p}^{12} \mathrm{P}$ \\
\hline 263 & $1 s^{2} 2 p^{2}\left({ }^{1} \mathrm{D}\right) 4 p^{1}$ & ${ }^{2} \mathrm{~F}$ & 3.5 & 12294467 & $76 \%+23 \% 1 \mathrm{~s}^{2} 2 \mathrm{p}^{2}\left({ }^{3} \mathrm{P}\right) 4 \mathrm{p}^{14} \mathrm{D}$ \\
\hline 264 & $1 \mathrm{~s}^{2} 2 \mathrm{p}^{2}\left({ }^{1} \mathrm{D}\right) 4 \mathrm{p}^{1}$ & ${ }^{2} \mathrm{D}$ & 2.5 & 12295436 & $58 \%+15 \% 1 \mathrm{~s}^{2} 2 \mathrm{p}^{2}\left({ }^{1} \mathrm{D}\right) 4 \mathrm{p}^{12} \mathrm{~F}+11 \% 1 \mathrm{~s}^{2} 2 \mathrm{p}^{2}\left({ }^{3} \mathrm{P}\right) 4 \mathrm{p}^{1{ }^{4} \mathrm{P}}$ \\
\hline 265 & $1 s^{2} 2 p^{2}\left({ }^{1} D\right) 4 p^{1}$ & ${ }^{2} \mathrm{P}$ & 0.5 & 12303528 & 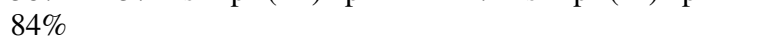 \\
\hline 266 & $1 s^{2} 2 s^{2} 5 p^{1}$ & ${ }^{2} \mathrm{P}$ & 0.5 & 12315762 & $95 \%$ \\
\hline 267 & $1 s^{2} 2 s^{2} 5 p^{1}$ & ${ }^{2} \mathrm{P}$ & 1.5 & 12322749 & $95 \%$ \\
\hline 268 & $1 s^{2} 2 p^{2}\left({ }^{1} \mathrm{D}\right) 4 \mathrm{p}^{1}$ & ${ }^{2} \mathrm{P}$ & 1.5 & 12331975 & $42 \%+23 \% 1 \mathrm{~s}^{2} 2 \mathrm{p}^{2}\left({ }^{1} \mathrm{D}\right) 4 \mathrm{p}^{12} \mathrm{D}+21 \% 1 \mathrm{~s}^{2} 2 \mathrm{p}^{2}\left({ }^{3} \mathrm{P}\right) 4 \mathrm{p}^{1}{ }^{2} \mathrm{P}$ \\
\hline 269 & $1 \mathrm{~s}^{2} 2 \mathrm{p}^{2}\left({ }^{1} \mathrm{D}\right) 4 \mathrm{~d}^{1}$ & ${ }^{2} \mathrm{~F}$ & 3.5 & 12345098 & $53 \%+24 \% 1 \mathrm{~s}^{2} 2 \mathrm{p}^{2}\left({ }^{1} \mathrm{D}\right) 4 \mathrm{~d}^{12} \mathrm{G}+13 \% 1 \mathrm{~s}^{2} 2 \mathrm{p}^{2}\left({ }^{3} \mathrm{P}\right) 4 \mathrm{~d}^{1}{ }^{4} \mathrm{D}$ \\
\hline 270 & $1 \mathrm{~s}^{2} 2 \mathrm{~s}^{2} 5 \mathrm{~d}^{1}$ & ${ }^{2} \mathrm{D}$ & 1.5 & 12351697 & $95 \%$ \\
\hline 271 & $1 \mathrm{~s}^{2} 2 \mathrm{p}^{2}\left({ }^{1} \mathrm{D}\right) 4 \mathrm{~d}^{1}$ & ${ }^{2} \mathrm{G}$ & 4.5 & 12352563 & $77 \%+22 \% 1 \mathrm{~s}^{2} 2 \mathrm{p}^{2}\left({ }^{3} \mathrm{P}\right) 4 \mathrm{~d}^{14} \mathrm{~F}$ \\
\hline 272 & $1 \mathrm{~s}^{2} 2 \mathrm{~s}^{2} 5 \mathrm{~d}^{1}$ & ${ }^{2} \mathrm{D}$ & 2.5 & 12353856 & $95 \%$ \\
\hline 273 & $1 \mathrm{~s}^{2} 2 \mathrm{p}^{2}\left({ }^{1} \mathrm{D}\right) 4 \mathrm{~d}^{1}$ & ${ }^{2} \mathrm{D}$ & 2.5 & 12359613 & $47 \%+27 \% 1 \mathrm{~s}^{2} 2 \mathrm{p}^{2}\left({ }^{1} \mathrm{D}\right) 4 \mathrm{~d}^{12} \mathrm{~F}+12 \% 1 \mathrm{~s}^{2} 2 \mathrm{p}^{2}\left({ }^{3} \mathrm{P}\right) 4 \mathrm{~d}^{14} \mathrm{D}$ \\
\hline 274 & $1 \mathrm{~s}^{2} 2 \mathrm{p}^{2}\left({ }^{1} \mathrm{D}\right) 4 \mathrm{~d}^{1}$ & ${ }^{2} \mathrm{D}$ & 1.5 & 12360467 & $69 \%$ \\
\hline 275 & $1 \mathrm{~s}^{2} 2 \mathrm{p}^{2}\left({ }^{1} \mathrm{D}\right) 4 \mathrm{~d}^{1}$ & ${ }^{2} \mathrm{G}$ & 3.5 & 12362665 & $44 \%+25 \% 1 \mathrm{~s}^{2} 2 \mathrm{p}^{2}\left({ }^{1} \mathrm{D}\right) 4 \mathrm{~d}^{12} \mathrm{~F}+18 \% 1 \mathrm{~s}^{2} 2 \mathrm{p}^{2}\left({ }^{3} \mathrm{P}\right) 4 \mathrm{~d}^{12} \mathrm{~F}$ \\
\hline 276 & $1 \mathrm{~s}^{2} 2 \mathrm{p}^{2}\left({ }^{1} \mathrm{D}\right) 4 \mathrm{~d}^{1}$ & ${ }^{2} \mathrm{P}$ & 0.5 & 12364441 & $79 \%$ \\
\hline 277 & $1 s^{2} 2 s^{2} 5 f^{1}$ & ${ }^{2} \mathrm{~F}$ & 2.5 & 12370344 & $95 \%$ \\
\hline 278 & $1 s^{2} 2 s^{2} 5 f^{1}$ & ${ }^{2} \mathrm{~F}$ & 3.5 & 12371272 & $95 \%$ \\
\hline 279 & $1 s^{2} 2 s^{2} 5 g^{1}$ & ${ }^{2} \mathrm{G}$ & 3.5 & 12374527 & $95 \%$ \\
\hline 280 & $1 \mathrm{~s}^{2} 2 \mathrm{~s}^{2} 5 \mathrm{~g}^{1}$ & ${ }^{2} \mathrm{G}$ & 4.5 & 12375073 & $95 \%$ \\
\hline 281 & $1 \mathrm{~s}^{2} 2 \mathrm{p}^{2}\left({ }^{1} \mathrm{D}\right) 4 \mathrm{~d}^{1}$ & ${ }^{2} \mathrm{~S}$ & 0.5 & 12379477 & $72 \%+16 \% 1 \mathrm{~s}^{2} 2 \mathrm{p}^{2}\left({ }^{3} \mathrm{P}\right) 4 \mathrm{~d}^{14} \mathrm{P}$ \\
\hline 282 & $1 \mathrm{~s}^{2} 2 \mathrm{p}^{2}\left({ }^{1} \mathrm{D}\right) 4 \mathrm{~d}^{1}$ & ${ }^{2} \mathrm{~F}$ & 2.5 & 12380298 & $36 \%+26 \% 1 \mathrm{~s}^{2} 2 \mathrm{p}^{2}\left({ }^{1} \mathrm{D}\right) 4 \mathrm{~d}^{12} \mathrm{D}+24 \% 1 \mathrm{~s}^{2} 2 \mathrm{p}^{2}\left({ }^{3} \mathrm{P}\right) 4 \mathrm{~d}^{12} \mathrm{D}$ \\
\hline 283 & $1 \mathrm{~s}^{2} 2 \mathrm{p}^{2}\left({ }^{1} \mathrm{D}\right) 4 \mathrm{f}^{1}$ & ${ }^{2} \mathrm{G}$ & 4.5 & 12383167 & $72 \%+19 \% 1 \mathrm{~s}^{2} 2 \mathrm{p}^{2}\left({ }^{3} \mathrm{P}\right) 4 \mathrm{f}^{1}{ }^{4} \mathrm{~F}$ \\
\hline 284 & $1 \mathrm{~s}^{2} 2 \mathrm{p}^{2}\left({ }^{1} \mathrm{D}\right) 4 \mathrm{f}^{1}$ & ${ }^{2} \mathrm{G}$ & 3.5 & 12383475 & $56 \%+15 \% 1 \mathrm{~s}^{2} 2 \mathrm{p}^{2}\left({ }^{1} \mathrm{D}\right) 4 \mathrm{f}^{1}{ }^{12} \mathrm{~F}+12 \% 1 \mathrm{~s}^{2} 2 \mathrm{p}^{2}\left({ }^{3} \mathrm{P}\right) 4 \mathrm{f}^{1}{ }^{4} \mathrm{~F}$ \\
\hline 285 & $1 \mathrm{~s}^{2} 2 \mathrm{p}^{2}\left({ }^{1} \mathrm{D}\right) 4 \mathrm{f}^{1}$ & ${ }^{2} \mathrm{~F}$ & 2.5 & 12385035 & $70 \%+11 \% 1 \mathrm{~s}^{2} 2 \mathrm{p}^{2}\left({ }^{3} \mathrm{P}\right) 4 \mathrm{f}^{14} \mathrm{~F}$ \\
\hline 286 & $1 \mathrm{~s}^{2} 2 \mathrm{p}^{2}\left({ }^{1} \mathrm{D}\right) 4 \mathrm{~d}^{1}$ & ${ }^{2} \mathrm{P}$ & 1.5 & 12385131 & $68 \%$ \\
\hline 287 & $1 \mathrm{~s}^{2} 2 \mathrm{p}^{2}\left({ }^{1} \mathrm{D}\right) 4 \mathrm{f}^{1}$ & ${ }^{2} \mathrm{~F}$ & 3.5 & 12387472 & $57 \%+14 \% 1 \mathrm{~s}^{2} 2 \mathrm{p}^{2}\left({ }^{1} \mathrm{D}\right) 4 \mathrm{f}^{12} \mathrm{G}+13 \% 1 \mathrm{~s}^{2} 2 \mathrm{p}^{2}\left({ }^{3} \mathrm{P}\right) 4 \mathrm{f}^{12} \mathrm{~F}$ \\
\hline 288 & $1 \mathrm{~s}^{2} 2 \mathrm{p}^{2}\left({ }^{1} \mathrm{D}\right) 4 \mathrm{f}^{1}$ & ${ }^{2} \mathrm{H}$ & 4.5 & 12389246 & $76 \%+17 \% 1 \mathrm{~s}^{2} 2 \mathrm{p}^{2}\left({ }^{3} \mathrm{P}\right) 4 \mathrm{f}^{1}{ }^{2} \mathrm{G}$ \\
\hline 289 & $1 \mathrm{~s}^{2} 2 \mathrm{p}^{2}\left({ }^{1} \mathrm{D}\right) 4 \mathrm{f}^{1}$ & ${ }^{2} \mathrm{H}$ & 5.5 & 12390193 & $77 \%+23 \% 1 \mathrm{~s}^{2} 2 \mathrm{p}^{2}\left({ }^{3} \mathrm{P}\right) 4 \mathrm{f}^{14} \mathrm{G}$ \\
\hline 290 & $1 \mathrm{~s}^{2} 2 \mathrm{p}^{2}\left({ }^{1} \mathrm{D}\right) 4 \mathrm{f}^{1}$ & ${ }^{2} \mathrm{D}$ & 1.5 & 12391428 & $76 \%$ \\
\hline 291 & $1 \mathrm{~s}^{2} 2 \mathrm{p}^{2}\left({ }^{1} \mathrm{D}\right) 4 \mathrm{f}^{1}$ & ${ }^{2} \mathrm{D}$ & 2.5 & 12394627 & $74 \%+10 \% 1 \mathrm{~s}^{2} 2 \mathrm{p}^{2}\left({ }^{3} \mathrm{P}\right) 4 \mathrm{f}^{1{ }^{4} \mathrm{D}}$ \\
\hline 292 & $1 \mathrm{~s}^{2} 2 \mathrm{p}^{2}\left({ }^{1} \mathrm{D}\right) 4 \mathrm{f}^{1}$ & ${ }^{2} \mathrm{P}$ & 0.5 & 12399188 & $79 \%+20 \% 1 \mathrm{~s}^{2} 2 \mathrm{p}^{2}\left({ }^{3} \mathrm{P}\right) 4 \mathrm{f}^{1{ }^{4} \mathrm{D}}$ \\
\hline 293 & $1 \mathrm{~s}^{2} 2 \mathrm{p}^{2}\left({ }^{1} \mathrm{D}\right) 4 \mathrm{f}^{1}$ & ${ }^{2} \mathrm{P}$ & 1.5 & 12400743 & $79 \%+11 \% 1 \mathrm{~s}^{2} 2 \mathrm{p}^{2}\left({ }^{3} \mathrm{P}\right) 4 \mathrm{f}^{12} \mathrm{D}$ \\
\hline 294 & $1 s^{2} 2 p^{2}\left({ }^{1} S\right) 4 s^{1}$ & ${ }^{2} \mathrm{~S}$ & 0.5 & 12444475 & $86 \%$ \\
\hline 295 & $1 s^{2} 2 p^{2}\left({ }^{1} S\right) 4 p^{1}$ & ${ }^{2} \mathrm{P}$ & 0.5 & 12515791 & $88 \%$ \\
\hline 296 & $1 s^{2} 2 p^{2}\left({ }^{1} S\right) 4 p^{1}$ & ${ }^{2} \mathrm{P}$ & 1.5 & 12525285 & $89 \%$ \\
\hline 297 & $1 \mathrm{~s}^{2} 2 \mathrm{p}^{2}\left({ }^{1} \mathrm{~S}\right) 4 \mathrm{~d}^{1}$ & ${ }^{2} \mathrm{D}$ & 2.5 & 12584193 & $89 \%$ \\
\hline 298 & $1 \mathrm{~s}^{2} 2 \mathrm{p}^{2}\left({ }^{1} \mathrm{~S}\right) 4 \mathrm{~d}^{1}$ & ${ }^{2} \mathrm{D}$ & 1.5 & 12585234 & $88 \%$ \\
\hline
\end{tabular}


Table 1. continued.

\begin{tabular}{|c|c|c|c|c|c|}
\hline Index & Configuration & $L S$ & $J$ & $\left(\mathrm{~cm}^{-1}\right)$ & ion \\
\hline 299 & $1 \mathrm{~s}^{2} 2 \mathrm{p}^{2}\left({ }^{1} \mathrm{~S}\right)$ & ${ }^{2} \mathrm{~F}$ & .5 & 612343 & $9 \%$ \\
\hline 300 & $1 \mathrm{~s}^{2} 2 \mathrm{p}^{2}\left({ }^{1} \mathrm{~S}\right) 4 \mathrm{f}^{1}$ & ${ }^{2} \mathrm{~F}$ & .5 & 762 & $\%$ \\
\hline 301 & $1 s^{2} 2 s^{1} 2 p^{1}\left({ }^{3} P\right) 5 s^{1}$ & ${ }^{4} \mathrm{P}$ & 0.5 & 2630518 & $80 \%+20 \% 1 \mathrm{~s}^{2} 2 \mathrm{~s}^{1} 2 \mathrm{p}^{1}\left({ }^{3} \mathrm{P}\right) 5 \mathrm{~s}^{1}{ }^{2} \mathrm{P}$ \\
\hline 302 & $1 s^{2} 2 s^{1} 2 p^{1}\left({ }^{3} P\right) 5 s^{1}$ & ${ }^{4} \mathrm{P}$ & 1.5 & 2659932 & $84 \%+13 \% 1 s^{2} 2 s^{1} 2 p^{1}\left({ }^{3} \mathrm{P}\right) 5 s^{12} \mathrm{P}$ \\
\hline 303 & $1 s^{2} 2 s^{1} 2 p^{1}\left({ }^{3} P\right) 5 p^{1}$ & ${ }^{4} \mathrm{D}$ & 0.5 & 2664345 & $68 \%+20 \% 1 \mathrm{~s}^{2} 2 \mathrm{~s}^{1} 2 \mathrm{p}^{1}\left({ }^{3} \mathrm{P}\right) 5 \mathrm{p}^{12} \mathrm{P}$ \\
\hline 304 & $1 s^{2} 2 s^{1} 2 p^{1}\left({ }^{3} P\right) 5 s^{1}$ & ${ }^{2} \mathrm{P}$ & 0.5 & 2668513 & $78 \%+19 \% 1 \mathrm{~s}^{2} 2 \mathrm{~s}^{1} 2 \mathrm{p}^{1}\left({ }^{3} \mathrm{P}\right) 5 \mathrm{~s}^{14} \mathrm{P}$ \\
\hline 305 & $1 s^{2} 2 s^{1} 2 p^{1}\left({ }^{3} P\right) 5 p^{1}$ & ${ }^{4} \mathrm{D}$ & 1.5 & 2674610 & $39 \%+29 \% 1 s^{2} 2 s^{1} 2 p^{1}\left({ }^{3} \mathrm{P}\right) 5 p^{14} \mathrm{P}+18 \% 1 \mathrm{~s}^{2} 2 \mathrm{~s}^{1} 2 \mathrm{p}^{1}\left({ }^{3} \mathrm{P}\right) 5 \mathrm{p}^{1}{ }^{2} \mathrm{D}$ \\
\hline 306 & $1 s^{2} 2 s^{1} 2 p^{1}\left({ }^{3} P\right) 5 p^{1}$ & ${ }^{4} \mathrm{D}$ & 1.5 & 2694215 & $46 \%+27 \% 1 s^{2} 2 s^{1} 2 p^{1}\left({ }^{3} \mathrm{P}\right) 5 p^{12} \mathrm{P}+13 \% 1 \mathrm{~s}^{2} 2 \mathrm{~s}^{1} 2 \mathrm{p}^{1}\left({ }^{3} \mathrm{P}\right) 5 \mathrm{p}^{1}{ }^{4} \mathrm{~s}$ \\
\hline 307 & $1 s^{2} 2 s^{1} 2 p^{1}\left({ }^{3} P\right) 5 d^{1}$ & ${ }^{4} \mathrm{~F}$ & 1.5 & 2699708 & $65 \%+14 \% 1 \mathrm{~s}^{2} 2 \mathrm{~s}^{1} 2 \mathrm{p}^{1}\left({ }^{3} \mathrm{P}\right) 5 \mathrm{~d}^{14} \mathrm{D}+12 \% 1 \mathrm{~s}^{2} 2 \mathrm{~s}^{1} 2 \mathrm{p}^{1}\left({ }^{3} \mathrm{P}\right) 5 \mathrm{~d}^{12} \mathrm{D}$ \\
\hline 308 & $1 s^{2} 2 s^{1} 2 p^{1}\left({ }^{3} P\right) 5 p^{1}$ & ${ }^{4} \mathrm{P}$ & 0.5 & 2702390 & $79 \%$ \\
\hline 309 & $1 s^{2} 2 s^{1} 2 p^{1}\left({ }^{3} \mathrm{P}\right) 5 p^{1}$ & ${ }^{4} \mathrm{D}$ & 2.5 & 2704007 & $63 \%+23 \% 1 s^{2} 2 s^{1} 2 p^{1}\left({ }^{3} P\right) 5 p^{14} P+12 \% 1 s^{2} 2 s^{1} 2 p^{1}\left({ }^{3} P\right) 5 p^{1}{ }^{2} D$ \\
\hline 310 & $1 s^{2} 2 s^{1} 2 p^{1}\left({ }^{3} P\right) 5 d^{1}$ & ${ }^{4} \mathrm{~F}$ & 2.5 & 2705133 & $31 \%+30 \% 1 \mathrm{~s}^{2} 2 \mathrm{~s}^{1} 2 \mathrm{p}^{1}\left({ }^{3} \mathrm{P}\right) 5 \mathrm{~d}^{14} \mathrm{D}+21 \% 1 \mathrm{~s}^{2} 2 \mathrm{~s}^{1} 2 \mathrm{p}^{1}\left({ }^{3} \mathrm{P}\right) 5 \mathrm{~d}^{14} \mathrm{P}$ \\
\hline 311 & $1 s^{2} 2 s^{1} 2 p^{1}\left({ }^{3} P\right) 5 p^{1}$ & ${ }^{2} \mathrm{P}$ & 0.5 & 2706122 & $52 \%+24 \% 1 \mathrm{~s}^{2} 2 \mathrm{~s}^{1} 2 \mathrm{p}^{1}\left({ }^{3} \mathrm{P}\right) 5 \mathrm{p}^{1{ }^{4} \mathrm{D}+}$ \\
\hline 312 & $1 s^{2} 2 s^{1} 2 p^{1}\left({ }^{3} P\right) 5 p^{1}$ & ${ }^{2} \mathrm{D}$ & 1.5 & 2709631 & $56 \%+20 \% 1 s^{2} 2 s^{1} 2 p^{1}\left({ }^{3} P\right) 5 p^{14} S+19 \% 1 s^{2} 2 s^{1} 2 p^{1}\left({ }^{3} P\right) 5 p^{1}{ }^{4} P$ \\
\hline 313 & $1 \mathrm{~s}^{2} 2 \mathrm{~s}^{1} 2 \mathrm{p}^{1}\left({ }^{3} \mathrm{P}\right) 5 \mathrm{f}^{1}$ & ${ }^{4} \mathrm{G}$ & 2.5 & 19581 & $47 \%+20 \% 1 \mathrm{~s}^{2} 2 \mathrm{~s}^{1} 2 \mathrm{p}^{1}\left({ }^{3} \mathrm{P}\right) 5 \mathrm{f}^{14} \mathrm{~F}+15 \% 1 \mathrm{~s}^{2} 2 \mathrm{~s}^{1} 2 \mathrm{p}^{1}\left({ }^{3} \mathrm{~F}\right.$ \\
\hline 314 & $1 \mathrm{~s}^{2} 2 \mathrm{~s}^{1} 2 \mathrm{p}^{1}\left({ }^{3} \mathrm{P}\right) 5 \mathrm{f}^{1}$ & ${ }^{4} \mathrm{~F}$ & 3.5 & 720793 & $28 \%+24 \% 1 \mathrm{~s}^{2} 2 \mathrm{~s}^{1} 2 \mathrm{p}^{1}\left({ }^{3} \mathrm{P}\right) 5 \mathrm{f}^{14} \mathrm{D}+22 \% 1 \mathrm{~s}^{2} 2 \mathrm{~s}^{1} 2 \mathrm{p}^{1}\left({ }^{3} \mathrm{P}\right) 5 \mathrm{f}^{1}{ }^{2} \mathrm{G}$ \\
\hline 315 & $1 \mathrm{~s}^{2} 2 \mathrm{~s}^{1} 2 \mathrm{p}^{1}\left({ }^{3} \mathrm{P}\right) 5 \mathrm{~g}^{1}$ & ${ }^{4} \mathrm{H}$ & 3. & 23256 & $41 \%+20 \% 1 \mathrm{~s}^{2} 2 \mathrm{~s}^{1} 2 \mathrm{p}^{1}\left({ }^{3} \mathrm{P}\right) 5 \mathrm{~g}^{1}{ }^{4} \mathrm{G}+18 \% 1 \mathrm{~s}^{2} 2 \mathrm{~s}^{1} 2 \mathrm{p}^{1}\left({ }^{3} \mathrm{P}\right) 5 \mathrm{~g}^{12} \mathrm{~F}$ \\
\hline 316 & $1 \mathrm{~s}^{2} 2 \mathrm{~s}^{1} 2 \mathrm{p}^{1}\left({ }^{3} \mathrm{P}\right) 5 \mathrm{~g}^{1}$ & ${ }^{4} \mathrm{G}$ & 4. & 812 & $25 \%+25 \% 1 \mathrm{~s}^{2} 2 \mathrm{~s}^{1} 2 \mathrm{p}^{1}\left({ }^{3} \mathrm{P}\right) 5 \mathrm{~g}^{14} \mathrm{~F}+25 \% 1 \mathrm{~s}^{2} 2 \mathrm{~s}^{1} 2 \mathrm{p}^{1}\left({ }^{3} \mathrm{~F}\right.$ \\
\hline 317 & $1 s^{2} 2 s^{1} 2 p^{1}\left({ }^{3} P\right) 5 d^{1}$ & ${ }^{4} \mathrm{~F}$ & 2. & & $49 \%+24 \% 1 \mathrm{~s}^{2} 2 \mathrm{~s}^{1} 2 \mathrm{p}^{1}\left({ }^{3} \mathrm{P}\right) 5 \mathrm{~d}^{12} \mathrm{D}+23 \% 1 \mathrm{~s}^{2} 2 \mathrm{~s}^{1} 2 \mathrm{p}^{1}{ }^{3}$ \\
\hline 318 & $1 \mathrm{~s}^{2} 2 \mathrm{~s}^{1} 2 \mathrm{p}^{1}\left({ }^{3} \mathrm{P}\right)$ & ${ }^{4} \mathrm{D}$ & 1. & & $44 \%+29 \% 1 \mathrm{~s}^{2} 2 \mathrm{~s}^{1} 2 \mathrm{p}^{1}\left({ }^{3} \mathrm{P}\right) 5 \mathrm{~d}^{14} \mathrm{P}$ \\
\hline 319 & $1 s^{2} 2 s^{1} 2 p^{1}\left({ }^{3} P\right)$ & ${ }^{4} \mathrm{~F}$ & 3. & 33667 & $55 \%+32 \% 1 \mathrm{~s}^{2} 2 \mathrm{~s}^{1} 2 \mathrm{p}^{1}\left({ }^{3} \mathrm{P}\right) 5 \mathrm{~d}^{14} \mathrm{D}+$ \\
\hline 320 & $1 s^{2} 2 s^{1} 2 p^{1}\left({ }^{3} P\right) 5 d^{1}$ & ${ }^{4} \mathrm{D}$ & 0 & 35488 & $77 \%+12 \% 1 \mathrm{~s}^{2} 2 \mathrm{~s}^{1} 2 \mathrm{p}^{1}\left({ }^{3} \mathrm{P}\right) 5 \mathrm{~d}^{12} \mathrm{P}$ \\
\hline 321 & $1 s^{2} 2 s^{1} 2 p^{1}\left({ }^{3} \mathrm{P}\right)$ & ${ }^{2} \mathrm{D}$ & 1. & & $40 \%+38 \% 1 \mathrm{~s}^{2} 2 \mathrm{~s}^{1} 2 \mathrm{p}^{1}\left({ }^{3} \mathrm{P}\right.$ \\
\hline 322 & $1 \mathrm{~s}^{2} 2 \mathrm{~s}^{1} 2 \mathrm{p}^{1}\left({ }^{3} \mathrm{P}\right)$ & ${ }^{2} \mathrm{~F}$ & 2 & & $59 \%+18 \% 1 \mathrm{~s}^{2} 2 \mathrm{~s}^{1} 2 \mathrm{p}^{1}\left({ }^{3} \mathrm{H}\right.$ \\
\hline 323 & $1 \mathrm{~s}^{2} 2 \mathrm{~s}^{1} 2 \mathrm{p}^{1}\left({ }^{3} \mathrm{P}\right) 5 \mathrm{f}^{1}$ & ${ }^{4} \mathrm{~F}$ & 1. & & $61 \mathrm{~s}^{2} 2 \mathrm{~s}^{1} 2 \mathrm{p}^{1}\left({ }^{3} \mathrm{P}\right.$ \\
\hline 324 & $1 s^{2} 2 s^{1} 2 p^{1}\left({ }^{3} P\right)$ & ${ }^{4} \mathrm{G}$ & 3. & & $49 \%+29 \% 1 \mathrm{~s}^{2} 2 \mathrm{~s}^{1} 2 \mathrm{p}^{1}\left({ }^{3} \mathrm{P}\right.$ \\
\hline 325 & $1 \mathrm{~s}^{2} 2 \mathrm{~s}^{1} 2 \mathrm{p}^{1}\left({ }^{3} \mathrm{P}\right)$ & ${ }^{4} \mathrm{G}$ & 4. & & $44 \%+38 \% 1 \mathrm{~s}^{2} 2 \mathrm{~s}^{1} 2 \mathrm{p}^{1}\left({ }^{3} \mathrm{P}\right.$ \\
\hline 326 & $1 \mathrm{~s}^{2} 2 \mathrm{~s}^{1} 2 \mathrm{p}^{1}\left({ }^{3} \mathrm{P}\right)$ & ${ }^{4} \mathrm{D}$ & . & & $33 \%+32 \% 1 \mathrm{~s}^{2} 2 \mathrm{~s}^{1} 2 \mathrm{p}^{1}\left({ }^{3} \mathrm{P}\right.$ \\
\hline 327 & $1 s^{2} 2 s^{1} 2 p^{1}\left({ }^{3} P\right)$ & ${ }^{4} \mathrm{P}$ & & & \\
\hline 328 & $1 \mathrm{~s}^{2} 2 \mathrm{~s}^{1} 2 \mathrm{p}^{1}\left({ }^{3} \mathrm{P}\right.$ & ${ }^{2} \mathrm{D}$ & 2 & & $44 \%+33 \% 1 \mathrm{~s}^{2} 2 \mathrm{~s}^{1} 2 \mathrm{p}^{1}\left({ }^{3} \mathrm{H}\right.$ \\
\hline 329 & $1 \mathrm{~s}^{2} 2 \mathrm{~s}^{1} 2 \mathrm{p}^{1}\left({ }^{3} \mathrm{P}\right)$ & ${ }^{4} \mathrm{H}$ & 2 & & $38 \%+30 \% 1 \mathrm{~s}^{2} 2 \mathrm{~s}^{1} 2 \mathrm{p}^{1}\left({ }^{3} \mathrm{P}\right.$ \\
\hline 330 & $1 s^{2} 2 s^{1} 2 p^{1}\left({ }^{3} \mathrm{P}\right)$ & ${ }^{2} \mathrm{G}$ & J. & & $47 \%+37 \% 1 \mathrm{~s}^{2} 2 \mathrm{~s}^{1} 2 \mathrm{p}^{1}\left({ }^{3} \mathrm{H}\right.$ \\
\hline 331 & $1 \mathrm{~s}^{2} 2 \mathrm{~s}^{1} 2 \mathrm{p}^{1}\left({ }^{3} \mathrm{P}\right)$ & ${ }^{4} \mathrm{G}$ & 0 & & $62 \%+19 \% 1 \mathrm{~s}^{2} 2 \mathrm{~s}^{1} 2 \mathrm{p}^{1}\left({ }^{3} \mathrm{P}\right.$ \\
\hline 332 & $1 s^{2} 2 s^{1} 2 p^{1}\left({ }^{3} \mathrm{P}\right)$ & ${ }^{4} \mathrm{H}$ & I & & $42 \%+40 \% 1 \mathrm{~s}^{2} 2 \mathrm{~s}^{1} 2 \mathrm{p}^{1}\left({ }^{3} \mathrm{P}\right)$ \\
\hline 333 & $1 \mathrm{~s}^{2} 2 \mathrm{~s}^{1} 2 \mathrm{p}^{1}\left({ }^{3} \mathrm{P}\right)$ & ${ }^{2} \mathrm{G}$ & I & & $33 \%+31 \% 1 \mathrm{~s}^{2} 2 \mathrm{~s}^{1} 2 \mathrm{p}^{1}\left({ }^{3} \mathrm{P}\right)$ \\
\hline 334 & $1 s^{2} 2 s^{1} 2 p^{1}\left({ }^{3} P\right)$ & ${ }^{4} \mathrm{H}$ & $\mathrm{J}$ & & $46 \%+41 \% 1 \mathrm{~s}^{2} 2 \mathrm{~s}^{1} 2 \mathrm{p}^{1}\left({ }^{3} \mathrm{H}\right.$ \\
\hline 335 & $1 s^{2} 2 s^{1} 2 p^{1} \quad\left({ }^{3} \mathrm{P}\right)$ & ${ }^{4} \mathrm{~F}$ & 3 & & $1 s^{2} 2 s^{1} 2$ \\
\hline 336 & $1 s^{2} 2 s^{1} 2 p^{1}\left({ }^{3} P\right.$ & ${ }^{2} \mathrm{P}$ & & & ${ }^{4} \mathrm{P}$ \\
\hline 337 & $1 s^{2} 2 s^{1} 2 p^{1} \quad\left({ }^{3} \mathrm{P}\right)$ & ${ }^{4} \mathrm{P}$ & 1. & & $1 s^{2} 2 s^{1}$ \\
\hline 338 & $1 \mathrm{~s}^{2} 2 \mathrm{~s}^{1} 2 \mathrm{p}^{1}\left({ }^{3} \mathrm{P}\right)$ & ${ }^{4} \mathrm{P}$ & 2 & & $1 s^{2} 2 s^{1} 2$ \\
\hline 39 & $1 s^{2} 2 s^{1} 2 p^{1}($ & ${ }^{4} \mathrm{D}$ & & & \\
\hline ) & $1 \mathrm{~s}^{2} 2 \mathrm{~s}^{1} 2 \mathrm{p}^{1}\left({ }^{3} \mathrm{P}\right)$ & ${ }^{2} \mathrm{P}$ & 1. & & $1 s^{2} 2 s^{1} 2 p^{1}\left({ }^{3} P\right.$ \\
\hline & $1 s^{2} 2 s^{1} 2 p$ & ${ }^{2} \mathrm{D}$ & & & ${ }^{4} \mathrm{P}$ \\
\hline J & $1 \mathrm{~s}^{2} 2 \mathrm{~s}^{1} 2 \mathrm{p}^{1}\left({ }^{3} \mathrm{P}\right)$ & ${ }^{2} S$ & 0 & & $s^{2} 2 s^{1} 2$ \\
\hline 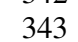 & $1 s^{2} 2 s^{1} 2 p$ & ${ }^{4} \mathrm{~F}$ & & & \\
\hline 344 & $1 \mathrm{~s}^{2} 2 \mathrm{~s}^{1} 2 \mathrm{p}^{1}\left({ }^{3} \mathrm{P}\right)$ & ${ }^{4} \mathrm{D}$ & 3 & & $s^{2} 2 s^{1} 2$ \\
\hline 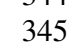 & $1 \mathrm{~s}^{2} 2 \mathrm{~s}^{1} 2 \mathrm{p}^{1}\left({ }^{3} \mathrm{P}\right)$ & ${ }^{4} \mathrm{D}$ & 2 & & ${ }^{14} \mathrm{~F}$ \\
\hline 6 & $1 \mathrm{~s}^{2} 2 \mathrm{~s}^{1} 2 \mathrm{p}^{1}\left({ }^{3} \mathrm{P}\right)$ & ${ }^{4} \mathrm{P}$ & 1 & & ${ }^{4} \mathrm{D}$ \\
\hline 347 & $1 \mathrm{~s}^{2} 2 \mathrm{~s}^{1} 2 \mathrm{p}^{1}\left({ }^{3} \mathrm{P}\right)$ & ${ }^{4} \mathrm{P}$ & 0 & & ${ }^{4} \mathrm{D}$ \\
\hline 210 & $1 \mathrm{~s}^{2} 2 \mathrm{~s}^{1} 2 \mathrm{p}^{1}\left({ }^{3} \mathrm{P}\right)$ & ${ }^{2} \mathrm{D}$ & 2 & & $2 s^{1} 2 p^{1}\left({ }^{3} \mathrm{P}\right.$ \\
\hline 0 & $1 \mathrm{~s}^{2} 2 \mathrm{~s}^{1} 2 \mathrm{p}^{1}\left({ }^{3} \mathrm{P}\right)$ & ${ }^{2} \mathrm{P}$ & & & ${ }^{14} \mathrm{P}$ \\
\hline 50 & $1 \mathrm{~s}^{2} 2 \mathrm{~s}^{1} 2 \mathrm{p}^{1}\left({ }^{3} \mathrm{P}\right)$ & ${ }^{2} \mathrm{~F}$ & & & \\
\hline 351 & $1 \mathrm{~s}^{2} 2 \mathrm{~s}^{1} 2 \mathrm{p}^{1}\left({ }^{3} \mathrm{P}\right)$ & ${ }^{4} \mathrm{~F}$ & 3. & & 20 \\
\hline 352 & $1 \mathrm{~s}^{2} 2 \mathrm{~s}^{1} 2 \mathrm{p}^{1}\left({ }^{3} \mathrm{P}\right) 5 \mathrm{f}^{1}$ & ${ }^{4} \mathrm{~F}$ & 4. & & $55 \%+$ \\
\hline 353 & $1 \mathrm{~s}^{2} 2 \mathrm{~s}^{1} 2 \mathrm{p}^{1}\left({ }^{3} \mathrm{P}\right) 5 \mathrm{f}^{1}$ & ${ }^{4} \mathrm{~F}$ & 2. & & ${ }^{4} \mathrm{D}$ \\
\hline 354 & $1 \mathrm{~s}^{2} 2 \mathrm{~s}^{1} 2 \mathrm{p}^{1}\left({ }^{3} \mathrm{P}\right) 5 \mathrm{~d}^{1}$ & ${ }^{2} \mathrm{P}$ & 0 . & & $87 \%+$ \\
\hline 355 & $1 \mathrm{~s}^{2} 2 \mathrm{~s}^{1} 2 \mathrm{p}^{1}\left({ }^{3} \mathrm{P}\right) 5 \mathrm{f}^{1}$ & ${ }^{4} \mathrm{G}$ & 5 & & $100 \%$ \\
\hline 356 & $1 \mathrm{~s}^{2} 2 \mathrm{~s}^{1} 2 \mathrm{p}^{1}\left({ }^{3} \mathrm{P}\right) 5 \mathrm{f}^{1}$ & ${ }^{2} \mathrm{~F}$ & 3. & & $52 \%+$ \\
\hline 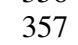 & $1 \mathrm{~s}^{2} 2 \mathrm{~s}^{1} 2 \mathrm{p}^{1}\left({ }^{3} \mathrm{P}\right) 5 \mathrm{f}^{1}$ & ${ }^{4} \mathrm{D}$ & 1. & & $70 \%+2$ \\
\hline 88 & $1 \mathrm{~s}^{2} 2 \mathrm{~s}^{1} 2 \mathrm{p}^{1}\left({ }^{3} \mathrm{P}\right) 5 \mathrm{f}^{1}$ & ${ }^{4} \mathrm{D}$ & 0 . & & $100 \%$ \\
\hline 59 & $1 \mathrm{~s}^{2} 2 \mathrm{~s}^{1} 2 \mathrm{p}^{1}\left({ }^{3} \mathrm{P}\right) 5 \mathrm{~g}^{1}$ & ${ }^{2} \mathrm{G}$ & 4.5 & 612 & $1 s^{2} 2 s^{1} 2 p^{1}\left(^{3}\right.$ \\
\hline
\end{tabular}


Table 1. continued.

\begin{tabular}{|c|c|c|c|c|c|}
\hline Index & Configuration & $L S$ & $J$ & $E\left(\mathrm{~cm}^{-1}\right)$ & Composition \\
\hline 360 & $1 \mathrm{~s}^{2} 2 \mathrm{~s}^{1} 2 \mathrm{p}^{1}\left({ }^{3} \mathrm{P}\right) 5 \mathrm{f}^{1}$ & ${ }^{2} \mathrm{G}$ & 4.5 & 12845829 & $85 \%$ \\
\hline 361 & $1 \mathrm{~s}^{2} 2 \mathrm{~s}^{1} 2 \mathrm{p}^{1}\left({ }^{3} \mathrm{P}\right) 5 \mathrm{f}^{1}$ & ${ }^{2} \mathrm{~F}$ & 2.5 & 12845954 & $36 \%+33 \% 1 \mathrm{~s}^{2} 2 \mathrm{~s}^{1} 2 \mathrm{p}^{1}\left({ }^{3} \mathrm{P}\right) 5 \mathrm{f}^{1}{ }^{2} \mathrm{D}+28 \% 1 \mathrm{~s}^{2} 2 \mathrm{~s}^{1} 2 \mathrm{p}^{1}\left({ }^{3} \mathrm{P}\right) 5 \mathrm{f}^{1}{ }^{4} \mathrm{D}$ \\
\hline 362 & $1 s^{2} 2 s^{1} 2 p^{1}\left({ }^{3} P\right) 5 g^{1}$ & ${ }^{4} \mathrm{G}$ & 3.5 & 12845979 & $42 \%+20 \% 1 \mathrm{~s}^{2} 2 \mathrm{~s}^{1} 2 \mathrm{p}^{1}\left({ }^{3} \mathrm{P}\right) 5 \mathrm{~g}^{12} \mathrm{G}+15 \% 1 \mathrm{~s}^{2} 2 \mathrm{~s}^{1} 2 \mathrm{p}^{1}\left({ }^{3} \mathrm{P}\right) 5 \mathrm{~g}^{12} \mathrm{~F}$ \\
\hline 363 & $1 s^{2} 2 s^{1} 2 p^{1}\left({ }^{3} \mathrm{P}\right) 5 \mathrm{~g}^{1}$ & ${ }^{4} \mathrm{G}$ & 5.5 & 12846054 & $57 \%+40 \% 1 \mathrm{~s}^{2} 2 \mathrm{~s}^{1} 2 \mathrm{p}^{1}\left({ }^{3} \mathrm{P}\right) 5 \mathrm{~g}^{1}{ }^{4} \mathrm{H}$ \\
\hline 364 & $1 s^{2} 2 s^{1} 2 p^{1}\left({ }^{3} \mathrm{P}\right) 5 \mathrm{~g}^{1}$ & ${ }^{4} \mathrm{G}$ & 4.5 & 12846473 & $32 \%+30 \% 1 \mathrm{~s}^{2} 2 \mathrm{~s}^{1} 2 \mathrm{p}^{1}\left({ }^{3} \mathrm{P}\right) 5 \mathrm{~g}^{1} \mathrm{C}+25 \% 1 \mathrm{~s}^{2} 2 \mathrm{~s}^{1} 2 \mathrm{p}^{1}\left({ }^{3} \mathrm{P}\right) 5 \mathrm{~g}^{14} \mathrm{~F}$ \\
\hline 365 & $1 s^{2} 2 s^{1} 2 p^{1}\left({ }^{3} \mathrm{P}\right) 5 \mathrm{~g}^{1}$ & ${ }^{2} \mathrm{H}$ & 5.5 & 12846865 & $81 \%+17 \% 1 \mathrm{~s}^{2} 2 \mathrm{~s}^{1} 2 \mathrm{p}^{1}\left({ }^{3} \mathrm{P}\right) 5 \mathrm{~g}^{1}{ }^{4} \mathrm{H}$ \\
\hline 366 & $1 s^{2} 2 s^{1} 2 p^{1}\left({ }^{3} \mathrm{P}\right) 5 \mathrm{~g}^{1}$ & ${ }^{4} \mathrm{~F}$ & 2.5 & 12847101 & $45 \%+35 \% 1 \mathrm{~s}^{2} 2 \mathrm{~s}^{1} 2 \mathrm{p}^{1}\left({ }^{3} \mathrm{P}\right) 5 \mathrm{~g}^{1}{ }^{4} \mathrm{G}+20 \% 1 \mathrm{~s}^{2} 2 \mathrm{~s}^{1} 2 \mathrm{p}^{1}\left({ }^{3} \mathrm{P}\right) 5 \mathrm{~g}^{12} \mathrm{~F}$ \\
\hline 367 & $1 s^{2} 2 s^{1} 2 p^{1}\left({ }^{3} \mathrm{P}\right) 5 \mathrm{~g}^{1}$ & ${ }^{4} \mathrm{H}$ & 6.5 & 12847235 & $100 \%$ \\
\hline 368 & $1 s^{2} 2 s^{1} 2 p^{1}\left({ }^{3} \mathrm{P}\right) 5 \mathrm{~g}^{1}$ & ${ }^{4} \mathrm{~F}$ & 3.5 & 12847598 & $43 \%+27 \% 1 \mathrm{~s}^{2} 2 \mathrm{~s}^{1} 2 \mathrm{p}^{1}\left({ }^{3} \mathrm{P}\right) 5 \mathrm{~g}^{1}{ }^{2} \mathrm{G}+21 \% 1 \mathrm{~s}^{2} 2 \mathrm{~s}^{1} 2 \mathrm{p}^{1}\left({ }^{3} \mathrm{P}\right) 5 \mathrm{~g}^{12} \mathrm{~F}$ \\
\hline 369 & $1 \mathrm{~s}^{2} 2 \mathrm{~s}^{1} 2 \mathrm{p}^{1}\left({ }^{3} \mathrm{P}\right) 5 \mathrm{~g}^{1}$ & ${ }^{4} \mathrm{~F}$ & 1.5 & 12848351 & $100 \%$ \\
\hline 370 & $1 s^{2} 2 s^{1} 2 p^{1}\left({ }^{3} P\right) 5 g^{1}$ & ${ }^{2} \mathrm{~F}$ & 2.5 & 12848953 & $64 \%+35 \% 1 \mathrm{~s}^{2} 2 \mathrm{~s}^{1} 2 \mathrm{p}^{1}\left({ }^{3} \mathrm{P}\right) 5 \mathrm{~g}^{1}{ }^{4} \mathrm{~F}$ \\
\hline 371 & $1 \mathrm{~s}^{2} 2 \mathrm{~s}^{1} 2 \mathrm{p}^{1}\left({ }^{3} \mathrm{P}\right) 5 \mathrm{f}^{1}$ & ${ }^{2} \mathrm{D}$ & 1.5 & 12849038 & $80 \%+15 \% 1 \mathrm{~s}^{2} 2 \mathrm{~s}^{1} 2 \mathrm{p}^{1}\left({ }^{3} \mathrm{P}\right) 5 \mathrm{f}^{14} \mathrm{D}$ \\
\hline 372 & $1 s^{2} 2 s^{2} 6 s^{1}$ & ${ }^{2} \mathrm{~S}$ & 0.5 & 12966803 & $94 \%$ \\
\hline 373 & $1 s^{2} 2 s^{2} 6 p^{1}$ & ${ }^{2} \mathrm{P}$ & 0.5 & 12988610 & $94 \%$ \\
\hline 374 & $1 s^{2} 2 s^{2} 6 p^{1}$ & ${ }^{2} \mathrm{P}$ & 1.5 & 12992885 & $94 \%$ \\
\hline 375 & $1 s^{2} 2 s^{2} 6 d^{1}$ & ${ }^{2} \mathrm{D}$ & 1.5 & 13010344 & $95 \%$ \\
\hline 376 & $1 s^{2} 2 s^{2} 6 d^{1}$ & ${ }^{2} \mathrm{D}$ & 2.5 & 13011577 & $95 \%$ \\
\hline 377 & $1 s^{2} 2 s^{2} 6 f^{1}$ & ${ }^{2} \mathrm{~F}$ & 2.5 & 13020767 & $95 \%$ \\
\hline 378 & $1 s^{2} 2 s^{2} 6 f^{1}$ & ${ }^{2} \mathrm{~F}$ & 3.5 & 13021353 & $96 \%$ \\
\hline 379 & $1 s^{2} 2 s^{2} 6 h^{1}$ & ${ }^{2} \mathrm{H}$ & 4.5 & 13022298 & $95 \%$ \\
\hline 380 & $1 s^{2} 2 s^{2} 6 g^{1}$ & ${ }^{2} \mathrm{G}$ & 3.5 & 13022462 & $95 \%$ \\
\hline 381 & $1 s^{2} 2 s^{2} 6 h^{1}$ & ${ }^{2} \mathrm{H}$ & 5.5 & 13022514 & $95 \%$ \\
\hline 382 & $1 s^{2} 2 s^{2} 6 g^{1}$ & ${ }^{2} \mathrm{G}$ & 4.5 & 13022792 & $95 \%$ \\
\hline 383 & $1 s^{2} 2 s^{1} 2 p^{1}\left({ }^{1} \mathrm{P}\right) 5 s^{1}$ & ${ }^{2} \mathrm{P}$ & 0.5 & 13048296 & $96 \%$ \\
\hline 384 & $1 s^{2} 2 s^{1} 2 p^{1}\left({ }^{1} \mathrm{P}\right) 5 s^{1}$ & ${ }^{2} \mathrm{P}$ & 1.5 & 13048997 & $96 \%$ \\
\hline 385 & $1 s^{2} 2 s^{1} 2 p^{1}\left({ }^{1} P\right) 5 p^{1}$ & ${ }^{2} \mathrm{D}$ & 1.5 & 13084306 & $82 \%+14 \% 1 \mathrm{~s}^{2} 2 \mathrm{~s}^{1} 2 \mathrm{p}^{1}\left({ }^{1} \mathrm{P}\right) 5 \mathrm{p}^{1}{ }^{2} \mathrm{P}$ \\
\hline 386 & $1 s^{2} 2 s^{1} 2 p^{1}\left({ }^{1} P\right) 5 p^{1}$ & ${ }^{2} \mathrm{P}$ & 0.5 & 13085077 & $89 \%$ \\
\hline 387 & $1 \mathrm{~s}^{2} 2 \mathrm{~s}^{1} 2 \mathrm{p}^{1}\left({ }^{1} \mathrm{P}\right) 5 \mathrm{p}^{1}$ & ${ }^{2} \mathrm{D}$ & 2.5 & 13089534 & $97 \%$ \\
\hline 388 & $1 s^{2} 2 s^{1} 2 p^{1}\left({ }^{1} P\right) 5 p^{1}$ & ${ }^{2} \mathrm{P}$ & 1.5 & 13091712 & $83 \%+14 \% 1 \mathrm{~s}^{2} 2 \mathrm{~s}^{1} 2 \mathrm{p}^{1}\left({ }^{1} \mathrm{P}\right) 5 \mathrm{p}^{12} \mathrm{D}$ \\
\hline 389 & $1 s^{2} 2 s^{1} 2 p^{1}\left({ }^{1} P\right) 5 p^{1}$ & ${ }^{2} \mathrm{~S}$ & 0.5 & 13097616 & $87 \%$ \\
\hline 390 & $1 \mathrm{~s}^{2} 2 \mathrm{~s}^{1} 2 \mathrm{p}^{1}\left({ }^{1} \mathrm{P}\right) 5 \mathrm{~d}^{1}$ & ${ }^{2} \mathrm{D}$ & 1.5 & 13119131 & $97 \%$ \\
\hline 391 & $1 \mathrm{~s}^{2} 2 \mathrm{~s}^{1} 2 \mathrm{p}^{1}\left({ }^{1} \mathrm{P}\right) 5 \mathrm{~d}^{1}$ & ${ }^{2} \mathrm{~F}$ & 2.5 & 13119873 & $88 \%$ \\
\hline 392 & $1 \mathrm{~s}^{2} 2 \mathrm{~s}^{1} 2 \mathrm{p}^{1}\left({ }^{1} \mathrm{P}\right) 5 \mathrm{~d}^{1}$ & ${ }^{2} \mathrm{~F}$ & 3.5 & 13120241 & $97 \%$ \\
\hline 393 & $1 s^{2} 2 s^{1} 2 p^{1}\left({ }^{1} \mathrm{P}\right) 5 d^{1}$ & ${ }^{2} \mathrm{D}$ & 2.5 & 13121768 & $88 \%$ \\
\hline 394 & $1 \mathrm{~s}^{2} 2 \mathrm{~s}^{1} 2 \mathrm{p}^{1}\left({ }^{1} \mathrm{P}\right) 5 \mathrm{~d}^{1}$ & ${ }^{2} \mathrm{P}$ & 0.5 & 13126394 & $97 \%$ \\
\hline 395 & $1 \mathrm{~s}^{2} 2 \mathrm{~s}^{1} 2 \mathrm{p}^{1}\left({ }^{1} \mathrm{P}\right) 5 \mathrm{~d}^{1}$ & ${ }^{2} \mathrm{P}$ & 1.5 & 13128371 & $97 \%$ \\
\hline 396 & $1 \mathrm{~s}^{2} 2 \mathrm{~s}^{1} 2 \mathrm{p}^{1}\left({ }^{1} \mathrm{P}\right) 5 \mathrm{f}^{1}$ & ${ }^{2} \mathrm{~F}$ & 2.5 & 13133808 & $97 \%$ \\
\hline 397 & $1 \mathrm{~s}^{2} 2 \mathrm{~s}^{1} 2 \mathrm{p}^{1}\left({ }^{1} \mathrm{P}\right) 5 \mathrm{f}^{1}$ & ${ }^{2} \mathrm{~F}$ & 3.5 & 13134781 & $97 \%$ \\
\hline 398 & $1 s^{2} 2 s^{1} 2 p^{1}\left({ }^{1} \mathrm{P}\right) 5 \mathrm{~g}^{1}$ & ${ }^{2} \mathrm{G}$ & 3.5 & 13138485 & $97 \%$ \\
\hline 399 & $1 \mathrm{~s}^{2} 2 \mathrm{~s}^{1} 2 \mathrm{p}^{1}\left({ }^{1} \mathrm{P}\right) 5 \mathrm{f}^{1}$ & ${ }^{2} \mathrm{G}$ & 3.5 & 13138756 & $97 \%$ \\
\hline 400 & $1 s^{2} 2 s^{1} 2 p^{1}\left({ }^{1} \mathrm{P}\right) 5 \mathrm{~g}^{1}$ & ${ }^{2} \mathrm{G}$ & 4.5 & 13139009 & $97 \%$ \\
\hline 401 & $1 \mathrm{~s}^{2} 2 \mathrm{~s}^{1} 2 \mathrm{p}^{1}\left({ }^{1} \mathrm{P}\right) 5 \mathrm{f}^{1}$ & ${ }^{2} \mathrm{G}$ & 4.5 & 13139549 & $97 \%$ \\
\hline 402 & $1 \mathrm{~s}^{2} 2 \mathrm{~s}^{1} 2 \mathrm{p}^{1}\left({ }^{1} \mathrm{P}\right) 5 \mathrm{f}^{1}$ & ${ }^{2} \mathrm{D}$ & 1.5 & 13141514 & $97 \%$ \\
\hline 403 & $1 \mathrm{~s}^{2} 2 \mathrm{~s}^{1} 2 \mathrm{p}^{1}\left({ }^{1} \mathrm{P}\right) 5 \mathrm{f}^{1}$ & ${ }^{2} \mathrm{D}$ & 2.5 & 13142299 & $97 \%$ \\
\hline 404 & $1 s^{2} 2 s^{1} 2 p^{1}\left({ }^{1} \mathrm{P}\right) 5 \mathrm{~g}^{1}$ & ${ }^{2} \mathrm{H}$ & 4.5 & 13142538 & $96 \%$ \\
\hline 405 & $1 s^{2} 2 s^{1} 2 p^{1}\left({ }^{1} \mathrm{P}\right) 5 \mathrm{~g}^{1}$ & ${ }^{2} \mathrm{H}$ & 5.5 & 13143067 & $97 \%$ \\
\hline 406 & $1 s^{2} 2 s^{1} 2 p^{1}\left({ }^{1} \mathrm{P}\right) 5 \mathrm{~g}^{1}$ & ${ }^{2} \mathrm{~F}$ & 2.5 & 13143102 & $97 \%$ \\
\hline 407 & $1 s^{2} 2 s^{1} 2 p^{1}\left({ }^{1} \mathrm{P}\right) 5 \mathrm{~g}^{1}$ & ${ }^{2} \mathrm{~F}$ & 3.5 & 13143620 & $97 \%$ \\
\hline
\end{tabular}


Table 2. Suggested change of spectroscopic identifications of levels to ensure the completeness of spectroscopic dataset. The indices of levels for which spectroscopic identifications are changed are presented in the first column. The second column contains index of level with the same highest contribution of configuration state function (Table 1) as level from the first column before change. Indices of levels in the first two columns are taken from Table 1.

\begin{tabular}{lll}
\hline \hline Index & Index & Changed CSF \\
\hline 88 & 81 & $2 \mathrm{p}^{2}\left({ }^{3} \mathrm{P}\right) 3 \mathrm{p}^{1}{ }^{2} \mathrm{D}_{2.5}$ \\
87 & 93 & $2 \mathrm{p}^{2}\left({ }^{3} \mathrm{P}\right) 3 \mathrm{~d}^{14} \mathrm{D}_{2.5}$ \\
117 & 109 & $2 \mathrm{p}^{2}\left({ }^{1} \mathrm{D}\right) 3 \mathrm{~d}^{1}{ }^{2} \mathrm{~F}_{3.5}$ \\
120 & 110 & $2 \mathrm{p}^{2}\left({ }^{1} \mathrm{D}\right) 3 \mathrm{~d}^{1}{ }^{2} \mathrm{~F}_{2.5}$ \\
152 & 175 & $2 \mathrm{~s}^{1} 2 \mathrm{p}^{1}\left({ }^{3} \mathrm{P}\right) 4 \mathrm{f}^{1}{ }^{4} \mathrm{D}_{3.5}$ \\
161 & 177 & $2 \mathrm{~s}^{1} 2 \mathrm{p}^{1}\left({ }^{3} \mathrm{P}\right) 4 \mathrm{f}^{1}{ }^{2} \mathrm{~F}_{2.5}$ \\
209 & 214 & $2 \mathrm{p}^{2}\left({ }^{3} \mathrm{P}\right) 4 \mathrm{p}^{1}{ }^{4} \mathrm{P}_{1.5}$ \\
224 & 213 & $2 \mathrm{p}^{2}\left({ }^{3} \mathrm{P}\right) 4 \mathrm{~d}^{1}{ }^{2} \mathrm{P}_{1.5}$ \\
228 & 215 & $2 \mathrm{p}^{2}\left({ }^{3} \mathrm{P}\right) 4 \mathrm{~d}^{1}{ }^{4} \mathrm{P}_{2.5}$ \\
219 & 233 & $2 \mathrm{p}^{2}\left({ }^{3} \mathrm{P}\right) 4 \mathrm{f}^{1}{ }^{2} \mathrm{D}_{2.5}$ \\
234 & 220 & $2 \mathrm{p}^{2}\left({ }^{3} \mathrm{P}\right) 4 \mathrm{f}^{1}{ }^{4} \mathrm{G}_{3.5}$ \\
306 & 305 & $2 \mathrm{~s}^{1} 2 \mathrm{p}^{1}\left({ }^{3} \mathrm{P}\right) 5 \mathrm{p}^{1}{ }^{4} \mathrm{~S}_{1.5}$ \\
310 & 317 & $2 \mathrm{~s}^{1} 2 \mathrm{p}^{1}\left({ }^{3} \mathrm{P}\right) 5 \mathrm{~d}^{14} \mathrm{P}_{2.5}$ \\
314 & 351 & $2 \mathrm{~s}^{1} 2 \mathrm{p}^{1}\left({ }^{3} \mathrm{P}\right) 5 \mathrm{f}^{1}{ }^{4} \mathrm{D}_{3.5}$ \\
315 & 334 & $2 \mathrm{~s}^{1} 2 \mathrm{p}^{1}\left({ }^{3} \mathrm{P}\right) 5 \mathrm{~g}^{1}{ }^{2} \mathrm{~F}_{3.5}$ \\
316 & 364 & $2 \mathrm{~s}^{1} 2 \mathrm{p}^{1}\left({ }^{3} \mathrm{P}\right) 5 \mathrm{~g}^{1}{ }^{2} \mathrm{H}_{4.5}$ \\
\hline
\end{tabular}


Table 3. Comparison of calculated energies $E^{\mathrm{GRASP}}$ and $E^{\mathrm{CITRO}}$ for Fe XXII levels with data compiled by NIST ( $E^{\mathrm{NIST}}$ ). Indices of levels in the first column and CSFs in the second column are taken from Table 1. Energies are in $\mathrm{cm}^{-1}$. The lifetimes of levels are presented in the last two columns.

\begin{tabular}{|c|c|c|c|c|c|c|}
\hline Index & CSF & $E^{\mathrm{NIST}}$ & $E^{\text {CITRO }}$ & $E^{\text {GRASP }}$ & $\tau^{\mathrm{CITRO}}(\mathrm{s})$ & $\tau^{\text {GRASP }}(\mathrm{s})$ \\
\hline 2 & $1 \mathrm{~s}^{2} 2 \mathrm{~s}^{2} 2 \mathrm{p}^{12} \mathrm{P}_{1.5}$ & 118266 & 115650 & 117953 & $7.19 \mathrm{E}-05$ & $6.81 \mathrm{E}-05$ \\
\hline 3 & $1 s^{2} 2 s^{1} 2 p^{2}{ }^{4} P_{0.5}$ & 404550 & 399846 & 402902 & $1.06 \mathrm{E}-08$ & $1.09 \mathrm{E}-08$ \\
\hline 4 & $1 \mathrm{~s}^{2} 2 \mathrm{~s}^{1} 2 \mathrm{p}^{2}{ }^{4} \mathrm{P}_{1.5}$ & 460190 & 454430 & 458031 & $9.81 \mathrm{E}-08$ & $1.02 \mathrm{E}-07$ \\
\hline 5 & $1 s^{2} 2 s^{1} 2 p^{2}{ }^{4} P_{2.5}$ & 513260 & 506869 & 511295 & $1.41 \mathrm{E}-08$ & $1.48 \mathrm{E}-08$ \\
\hline 6 & $1 s^{2} 2 s^{1} 2 p^{2}{ }^{2} D_{1.5}$ & 736310 & 728951 & 741538 & $9.49 \mathrm{E}-11$ & $9.13 \mathrm{E}-11$ \\
\hline 7 & $1 \mathrm{~s}^{2} 2 \mathrm{~s}^{1} 2 \mathrm{p}^{2}{ }^{2} \mathrm{D}_{2.5}$ & 759210 & 751725 & 763743 & $1.71 \mathrm{E}-10$ & $1.65 \mathrm{E}-10$ \\
\hline 8 & $1 \mathrm{~s}^{2} 2 \mathrm{~s}^{1} 2 \mathrm{p}^{2}{ }^{2} \mathrm{P}_{0.5}$ & 853650 & 847120 & 861921 & $2.64 \mathrm{E}-11$ & $2.55 \mathrm{E}-11$ \\
\hline 9 & $1 s^{2} 2 s^{1} 2 p^{2}{ }^{2} S_{0.5}$ & 978350 & 969456 & 986277 & $2.82 \mathrm{E}-11$ & $2.71 \mathrm{E}-11$ \\
\hline 10 & $1 s^{2} 2 s^{1} 2 p^{2}{ }^{2} P_{1.5}$ & 992320 & 983323 & 1000692 & $2.01 \mathrm{E}-11$ & $1.94 \mathrm{E}-11$ \\
\hline 11 & $1 \mathrm{~s}^{2} 2 \mathrm{p}^{3}{ }^{4} \mathrm{~S}_{1.5}$ & 1255700 & 1245542 & 1260179 & $2.29 \mathrm{E}-11$ & $2.22 \mathrm{E}-11$ \\
\hline 12 & $1 s^{2} 2 p^{3}{ }^{2} D_{1.5}$ & 1396110 & 1384720 & 1405688 & $4.69 \mathrm{E}-11$ & $4.61 \mathrm{E}-11$ \\
\hline 13 & $1 \mathrm{~s}^{2} 2 \mathrm{p}^{3}{ }^{2} \mathrm{D}_{2.5}$ & 1426570 & 1414255 & 1435674 & $4.89 \mathrm{E}-11$ & $4.77 \mathrm{E}-11$ \\
\hline 14 & $1 \mathrm{~s}^{2} 2 \mathrm{p}^{3}{ }^{2} \mathrm{P}_{0.5}$ & 1569630 & 1556753 & 1582452 & $2.33 \mathrm{E}-11$ & $2.24 \mathrm{E}-11$ \\
\hline 15 & $1 \mathrm{~s}^{2} 2 \mathrm{p}^{3}{ }^{2} \mathrm{P}_{1.5}$ & 1627720 & 1613294 & 1639226 & $2.38 \mathrm{E}-11$ & $2.30 \mathrm{E}-11$ \\
\hline 20 & $1 \mathrm{~s}^{2} 2 \mathrm{~s}^{2} 3 \mathrm{~d}^{12} \mathrm{D}_{1.5}$ & 8498000 & 8508732 & 8491152 & $5.10 \mathrm{E}-14$ & $5.12 \mathrm{E}-14$ \\
\hline 21 & $1 \mathrm{~s}^{2} 2 \mathrm{~s}^{2} 3 \mathrm{~d}^{12} \mathrm{D}_{2.5}$ & 8507000 & 8518565 & 8501013 & $5.26 \mathrm{E}-14$ & $5.31 \mathrm{E}-14$ \\
\hline 28 & $1 s^{2} 2 s^{1} 2 p^{1}\left({ }^{3} P\right) 3 p^{1}{ }^{2} P_{1.5}$ & 8688000 & 8707856 & 8695560 & $2.85 \mathrm{E}-13$ & $2.96 \mathrm{E}-13$ \\
\hline 29 & $1 s^{2} 2 s^{1} 2 p^{1}\left({ }^{3} \mathrm{P}\right) 3 p^{1}{ }^{2} \mathrm{P}_{0.5}$ & 8584000 & 8716292 & 8704356 & $1.35 \mathrm{E}-13$ & $1.38 \mathrm{E}-13$ \\
\hline 32 & $1 s^{2} 2 s^{1} 2 p^{1}\left({ }^{3} P\right) 3 p^{12} D_{1.5}$ & 8740000 & 8758943 & 8748534 & $1.94 \mathrm{E}-13$ & $1.99 \mathrm{E}-13$ \\
\hline 39 & $1 s^{2} 2 s^{1} 2 p^{1}\left({ }^{3} P\right) 3 p^{12} D_{2.5}$ & 8845000 & 8862704 & 8857624 & $1.30 \mathrm{E}-13$ & $1.35 \mathrm{E}-13$ \\
\hline 40 & $1 s^{2} 2 s^{1} 2 p^{1}\left({ }^{3} P\right) 3 d^{1}{ }^{4} F_{3.5}$ & 8864000 & 8870786 & 8857700 & $1.68 \mathrm{E}-13$ & $1.64 \mathrm{E}-13$ \\
\hline 41 & $1 \mathrm{~s}^{2} 2 \mathrm{~s}^{1} 2 \mathrm{p}^{1}\left({ }^{3} \mathrm{P}\right) 3 \mathrm{~d}^{1}{ }^{4} \mathrm{P}_{2.5}$ & 8874000 & 8882016 & 8868503 & $5.84 \mathrm{E}-14$ & $5.93 \mathrm{E}-14$ \\
\hline 42 & $1 s^{2} 2 s^{1} 2 p^{1}\left({ }^{3} P\right) 3 d^{14} D_{1.5}$ & 8882000 & 8890791 & 8878309 & $5.97 \mathrm{E}-14$ & $4.23 \mathrm{E}-14$ \\
\hline 43 & $1 s^{2} 2 s^{1} 2 p^{1}\left({ }^{3} P\right) 3 d^{14} D_{0.5}$ & 8888000 & 8895387 & 8881447 & $3.73 \mathrm{E}-14$ & $3.52 \mathrm{E}-14$ \\
\hline 49 & $1 s^{2} 2 s^{1} 2 p^{1}\left({ }^{3} P\right) 3 d^{12} D_{25}$ & 8938000 & 8940836 & 8930222 & $6.80 \mathrm{E}-14$ & $6.84 \mathrm{E}-14$ \\
\hline 50 & $1 \mathrm{~s}^{2} 2 \mathrm{~s}^{1} 2 \mathrm{p}^{1}\left({ }^{3} \mathrm{P}\right) 3 \mathrm{~d}^{1}{ }^{4} \mathrm{D}_{3.5}$ & 8962000 & 8965402 & 8955370 & $4.06 \mathrm{E}-14$ & $4.13 \mathrm{E}-14$ \\
\hline 51 & $1 \mathrm{~s}^{2} 2 \mathrm{~s}^{1} 2 \mathrm{p}^{1}\left({ }^{3} \mathrm{P}\right) 3 \mathrm{~d}^{1}{ }^{4} \mathrm{D}_{2.5}$ & 8973000 & 8976048 & 8966348 & $4.40 \mathrm{E}-14$ & $4.44 \mathrm{E}-14$ \\
\hline 52 & $1 \mathrm{~s}^{2} 2 \mathrm{~s}^{1} 2 \mathrm{p}^{1}\left({ }^{3} \mathrm{P}\right) 3 \mathrm{~d}^{1}{ }^{4} \mathrm{P}_{1.5}$ & 8972000 & 8981681 & 8972247 & $4.90 \mathrm{E}-14$ & $4.94 \mathrm{E}-14$ \\
\hline 53 & $1 \mathrm{~s}^{2} 2 \mathrm{~s}^{1} 2 \mathrm{p}^{1}\left({ }^{3} \mathrm{P}\right) 3 \mathrm{~d}^{1}{ }^{4} \mathrm{P}_{0.5}$ & 8973000 & 8985011 & 8975643 & $5.65 \mathrm{E}-14$ & $5.70 \mathrm{E}-14$ \\
\hline 54 & $1 \mathrm{~s}^{2} 2 \mathrm{~s}^{1} 2 \mathrm{p}^{1}\left({ }^{3} \mathrm{P}\right) 3 \mathrm{~d}^{1}{ }^{2} \mathrm{~F}_{2.5}$ & 9030000 & 9012 & 9004790 & -14 & $\mathrm{E}-14$ \\
\hline 55 & $1 \mathrm{~s}^{2} 2 \mathrm{~s}^{1} 2 \mathrm{p}^{1}\left({ }^{3} \mathrm{P}\right) 3 \mathrm{~d}^{1}{ }^{2} \mathrm{P}_{1.5}$ & 9180000 & & 904 & -14 & $\mathrm{E}-14$ \\
\hline 56 & $1 \mathrm{~s}^{2} 2 \mathrm{~s}^{1} 2 \mathrm{p}^{1}\left({ }^{3} \mathrm{P}\right) 3 \mathrm{~d}^{1}{ }^{2} \mathrm{~F}_{3.5}$ & 9062000 & 9066838 & 9060831 & $4.45 \mathrm{E}-14$ & $4.42 \mathrm{E}-14$ \\
\hline 59 & $1 \mathrm{~s}^{2} 2 \mathrm{~s}^{1} 2 \mathrm{p}^{1}\left({ }^{3} \mathrm{P}\right) 3 \mathrm{~d}^{1}{ }^{2} \mathrm{P}_{0.5}$ & 8967000 & 9084039 & 9078984 & E-14 & $5.74 \mathrm{E}-14$ \\
\hline 66 & $1 \mathrm{~s}^{2} 2 \mathrm{~s}^{1} 2 \mathrm{p}^{1}\left({ }^{1} \mathrm{P}\right) 3 \mathrm{~d}^{1}{ }^{2} \mathrm{~F}_{3.5}$ & 9242000 & 924 & 9250872 & -14 & $E-14$ \\
\hline 67 & $1 \mathrm{~s}^{2} 2 \mathrm{~s}^{1} 2 \mathrm{p}^{1}\left({ }^{1} \mathrm{P}\right) 3 \mathrm{~d}^{1}{ }^{2} \mathrm{~F}_{2.5}$ & 9249000 & 925 & 925 & -14 & $4.91 \mathrm{E}-14$ \\
\hline 69 & $1 \mathrm{~s}^{2} 2 \mathrm{~s}^{1} 2 \mathrm{p}^{1}\left({ }^{1} \mathrm{P}\right) 3 \mathrm{~d}^{12} \mathrm{D}_{1.5}$ & 9134000 & 926 & 926 & -14 & $\mathrm{E}-14$ \\
\hline 71 & $1 \mathrm{~s}^{2} 2 \mathrm{~s}^{1} 2 \mathrm{p}^{1}\left({ }^{1} \mathrm{P}\right) 3 \mathrm{~d}^{1}{ }^{2} \mathrm{D}_{2.5}$ & 9272000 & 927 & 9276912 & -14 & $\mathrm{E}-14$ \\
\hline 74 & $1 \mathrm{~s}^{2} 2 \mathrm{~s}^{1} 2 \mathrm{p}^{1}\left({ }^{1} \mathrm{P}\right) 3 \mathrm{~d}^{1}{ }^{2} \mathrm{P}_{1.5}$ & 9168 & 929 & 9300838 & $6.00 \mathrm{E}-14$ & $6.26 \mathrm{E}-14$ \\
\hline 126 & $1 \mathrm{~s}^{2} 2 \mathrm{~s}^{2} 4 \mathrm{~s}^{1}{ }^{2} \mathrm{~S}_{0.5}$ & 00 & 1 & 1008 & $E-13$ & $5.83 \mathrm{E}-13$ \\
\hline 129 & $1 \mathrm{~s}^{2} 2 \mathrm{~s}^{2} 4 \mathrm{~d}^{12} \mathrm{D}_{1.5}$ & 0000 & 06 & 85 & $1.28 \mathrm{E}-13$ & $1.24 \mathrm{E}-13$ \\
\hline 130 & $1 \mathrm{~s}^{2} 2 \mathrm{~s}^{2} 4 \mathrm{~d}^{12} \mathrm{D}_{2.5}$ & 0000 & 02 & 49 & $\mathrm{E}-13$ & $1.26 \mathrm{E}-13$ \\
\hline 142 & $1 \mathrm{~s}^{2} 2 \mathrm{~s}^{1} 2 \mathrm{p}^{1}\left({ }^{3} \mathrm{P}\right) 4 \mathrm{p}^{1}{ }^{2} \mathrm{P}_{0.5}$ & 5000 & 11478060 & 11460571 & $2.70 \mathrm{E}-13$ & $2.47 \mathrm{E}-13$ \\
\hline 143 & $1 s^{2} 2 s^{1} 2 p^{1}\left({ }^{3} P\right) 4 p^{12} D_{1.5}$ & 11474000 & 11487446 & 11470801 & $2.70 \mathrm{E}-13$ & $2.64 \mathrm{E}-13$ \\
\hline 146 & $1 \mathrm{~s}^{2} 2 \mathrm{~s}^{1} 2 \mathrm{p}^{1}\left({ }^{3} \mathrm{P}\right) 4 \mathrm{~d}^{1}{ }^{4} \mathrm{~F}_{2.5}$ & 11492000 & 11509097 & 11489897 & $1.78 \mathrm{E}-13$ & $1.77 \mathrm{E}-13$ \\
\hline 149 & $1 \mathrm{~s}^{2} 2 \mathrm{~s}^{1} 2 \mathrm{p}^{1}\left({ }^{3} \mathrm{P}\right) 4 \mathrm{~d}^{14} \mathrm{D}_{1.5}$ & 11526000 & 536933 & 11518812 & $1.15 \mathrm{E}-13$ & $1.11 \mathrm{E}-13$ \\
\hline 156 & $1 \mathrm{~s}^{2} 2 \mathrm{~s}^{1} 2 \mathrm{p}^{1}\left({ }^{3} \mathrm{P}\right) 4 \mathrm{~d}^{1}{ }^{2} \mathrm{~F}_{2.5}$ & 11558000 & 11557332 & 11541402 & $1.23 \mathrm{E}-13$ & $1.18 \mathrm{E}-13$ \\
\hline 168 & $1 \mathrm{~s}^{2} 2 \mathrm{~s}^{1} 2 \mathrm{p}^{1}\left({ }^{3} \mathrm{P}\right) 4 \mathrm{~d}^{1}{ }^{4} \mathrm{D}_{3.5}$ & 11618000 & 11618692 & 11604622 & $1.26 \mathrm{E}-13$ & $1.22 \mathrm{E}-13$ \\
\hline 169 & $1 \mathrm{~s}^{2} 2 \mathrm{~s}^{1} 2 \mathrm{p}^{1}\left({ }^{3} \mathrm{P}\right) 4 \mathrm{~d}^{1}{ }^{4} \mathrm{D}_{2.5}$ & 11618000 & 11621954 & 11607936 & $1.16 \mathrm{E}-13$ & $1.12 \mathrm{E}-13$ \\
\hline 172 & $1 \mathrm{~s}^{2} 2 \mathrm{~s}^{1} 2 \mathrm{p}^{1}\left({ }^{3} \mathrm{P}\right) 4 \mathrm{~d}^{1}{ }^{2} \mathrm{D}_{2.5}$ & 11611000 & 11633161 & 11620801 & $1.43 \mathrm{E}-13$ & $1.38 \mathrm{E}-13$ \\
\hline 174 & $1 \mathrm{~s}^{2} 2 \mathrm{~s}^{1} 2 \mathrm{p}^{1}\left({ }^{3} \mathrm{P}\right) 4 \mathrm{~d}^{12} \mathrm{~F}_{3.5}$ & 11900000 & 11652015 & 11641117 & $9.77 \mathrm{E}-14$ & $9.30 \mathrm{E}-14$ \\
\hline 194 & $1 \mathrm{~s}^{2} 2 \mathrm{~s}^{1} 2 \mathrm{p}^{1}\left({ }^{1} \mathrm{P}\right) 4 \mathrm{~d}^{1}{ }^{2} \mathrm{~F}_{2.5}$ & 11897000 & 11903101 & 11903215 & $1.52 \mathrm{E}-13$ & $1.45 \mathrm{E}-13$ \\
\hline 195 & $1 \mathrm{~s}^{2} 2 \mathrm{~s}^{1} 2 \mathrm{p}^{1}\left({ }^{1} \mathrm{P}\right) 4 \mathrm{~d}^{12} \mathrm{~F}_{3.5}$ & 11649000 & 11902966 & 11903365 & $2.06 \mathrm{E}-13$ & $2.01 \mathrm{E}-13$ \\
\hline 196 & $1 \mathrm{~s}^{2} 2 \mathrm{~s}^{1} 2 \mathrm{p}^{1}\left({ }^{1} \mathrm{P}\right) 4 \mathrm{~d}^{1}{ }^{2} \mathrm{D}_{2.5}$ & 11906000 & 11908125 & 11908277 & $1.06 \mathrm{E}-13$ & $1.02 \mathrm{E}-13$ \\
\hline 270 & $1 \mathrm{~s}^{2} 2 \mathrm{~s}^{2} 5 \mathrm{~d}^{12} \mathrm{D}_{1.5}$ & 12359000 & 12377319 & 12351697 & $2.32 \mathrm{E}-13$ & $2.44 \mathrm{E}-13$ \\
\hline
\end{tabular}


V. Jonauskas et al.: Transition rates for Fe XXII, Online Material $p 11$

Table 4. Comparison of calculated wavelengths $\left(\lambda^{\mathrm{GRASP}}, \lambda^{\mathrm{CITRO}}\right)$ and line strengths $\left(S^{\mathrm{GRASP}}, S^{\mathrm{CITRO}}\right)$ for Fe XXII with values presented by NIST $\left(\lambda^{\text {NIST }}, S^{\text {NIST }}\right)$. Indices of levels in the first two columns are taken from Table 1.

\begin{tabular}{|c|c|c|c|c|c|c|c|}
\hline$k$ & $i$ & $\lambda^{\mathrm{NIST}}(\AA)$ & $\lambda^{\mathrm{CITRO}}(\AA)$ & $\lambda^{\mathrm{GRASP}}(\AA)$ & $S^{\text {NIST }}$ & $S^{\text {CITRO }}$ & $S^{\text {GRASP }}$ \\
\hline$\overline{1}$ & 2 & 845.52 & 864.68 & 847.80 & $1.33 \mathrm{E}+00$ & $1.33 \mathrm{E}+00$ & $1.33 \mathrm{E}+00$ \\
\hline 1 & 3 & 247.19 & 250.10 & 248.20 & $1.30 \mathrm{E}-03$ & $1.18 \mathrm{E}-03$ & $1.24 \mathrm{E}-03$ \\
\hline 1 & 6 & 135.81 & 137.18 & 134.85 & $5.40 \mathrm{E}-02$ & $5.29 \mathrm{E}-02$ & $5.36 \mathrm{E}-02$ \\
\hline 1 & 8 & 117.14 & 118.05 & 116.02 & $6.20 \mathrm{E}-02$ & $6.05 \mathrm{E}-02$ & $6.13 \mathrm{E}-02$ \\
\hline 1 & 9 & 102.22 & 103.15 & 101.39 & $2.80 \mathrm{E}-03$ & $2.28 \mathrm{E}-03$ & $2.32 \mathrm{E}-03$ \\
\hline 1 & 10 & 100.77 & 101.70 & 99.93 & $1.25 \mathrm{E}-02$ & $1.25 \mathrm{E}-02$ & $1.27 \mathrm{E}-02$ \\
\hline 1 & 20 & 11.77 & 11.75 & 11.78 & $5.10 \mathrm{E}-02$ & $5.28 \mathrm{E}-02$ & $5.27 \mathrm{E}-02$ \\
\hline 1 & 126 & 9.06 & 9.08 & 9.10 & $1.40 \mathrm{E}-04$ & $2.52 \mathrm{E}-04$ & $2.10 \mathrm{E}-004$ \\
\hline 1 & 129 & 8.98 & 8.96 & 8.98 & $6.60 \mathrm{E}-03$ & $7.61 \mathrm{E}-03$ & $7.26 \mathrm{E}-003$ \\
\hline 2 & 3 & 349.30 & 351.87 & 350.94 & $5.90 \mathrm{E}-04$ & $5.55 \mathrm{E}-04$ & $5.78 \mathrm{E}-04$ \\
\hline 2 & 4 & 292.46 & 295.18 & 294.05 & $4.20 \mathrm{E}-04$ & $3.98 \mathrm{E}-04$ & $4.16 \mathrm{E}-04$ \\
\hline 2 & 5 & 253.17 & 255.61 & 254.23 & $3.40 \mathrm{E}-03$ & $3.30 \mathrm{E}-03$ & $3.50 \mathrm{E}-03$ \\
\hline 2 & 6 & 161.74 & 163.05 & 160.36 & $3.20 \mathrm{E}-04$ & $2.64 \mathrm{E}-04$ & $2.46 \mathrm{E}-04$ \\
\hline 2 & 7 & 156.02 & 157.21 & 154.85 & $7.00 \mathrm{E}-02$ & $6.67 \mathrm{E}-02$ & $6.75 \mathrm{E}-02$ \\
\hline 2 & 8 & 136.01 & 136.71 & 134.41 & $3.00 \mathrm{E}-05$ & $1.65 \mathrm{E}-04$ & $1.97 \mathrm{E}-04$ \\
\hline 2 & 9 & 116.26 & 117.12 & 115.16 & $5.48 \mathrm{E}-02$ & $5.23 \mathrm{E}-02$ & $5.28 \mathrm{E}-02$ \\
\hline 2 & 10 & 114.41 & 115.25 & 113.28 & $1.30 \mathrm{E}-01$ & $1.30 \mathrm{E}-01$ & $1.32 \mathrm{E}-01$ \\
\hline 2 & 20 & 11.94 & 11.91 & 11.94 & $1.00 \mathrm{E}-02$ & $1.06 \mathrm{E}-02$ & $1.06 \mathrm{E}-02$ \\
\hline 2 & 21 & 11.92 & 11.91 & 11.93 & $9.00 \mathrm{E}-02$ & $9.46 \mathrm{E}-02$ & $9.48 \mathrm{E}-02$ \\
\hline 2 & 126 & 9.14 & 9.18 & 9.20 & $4.20 \mathrm{E}-04$ & $5.42 \mathrm{E}-04$ & $5.02 \mathrm{E}-04$ \\
\hline 2 & 129 & 9.07 & 9.06 & 9.08 & $1.40 \mathrm{E}-03$ & $1.54 \mathrm{E}-03$ & $1.47 \mathrm{E}-03$ \\
\hline 2 & 130 & 9.07 & 9.05 & 9.07 & $1.20 \mathrm{E}-02$ & $1.38 \mathrm{E}-02$ & $1.33 \mathrm{E}-02$ \\
\hline 3 & 11 & 117.52 & 118.25 & 116.65 & $3.30 \mathrm{E}-02$ & $3.20 \mathrm{E}-02$ & $3.24 \mathrm{E}-02$ \\
\hline 3 & 12 & 100.82 & 101.54 & 99.72 & $6.70 \mathrm{E}-05$ & $7.28 \mathrm{E}-05$ & $1.03 \mathrm{E}-04$ \\
\hline 3 & 14 & 85.83 & 86.44 & 84.78 & $1.40 \mathrm{E}-04$ & $1.30 \mathrm{E}-04$ & $1.32 \mathrm{E}-04$ \\
\hline 3 & 42 & 11.80 & 11.78 & 11.80 & $5.50 \mathrm{E}-02$ & $5.38 \mathrm{E}-02$ & $3.32 \mathrm{E}-02$ \\
\hline 3 & 43 & 11.79 & 11.78 & 11.79 & $4.20 \mathrm{E}-02$ & $4.31 \mathrm{E}-02$ & $4.11 \mathrm{E}-02$ \\
\hline 3 & 149 & 8.99 & 8.98 & 9.00 & $7.00 \mathrm{E}-03$ & $7.67 \mathrm{E}-03$ & $7.25 \mathrm{E}-03$ \\
\hline 4 & 11 & 125.71 & 126.40 & 24.67 & $5.96 \mathrm{E}-02$ & $5.76 \mathrm{E}-02$ & $5.81 \mathrm{E}-02$ \\
\hline 4 & 12 & 106.81 & 107.49 & 105.52 & $5.50 \mathrm{E}-03$ & $5.10 \mathrm{E}-03$ & $5.52 \mathrm{E}-03$ \\
\hline 4 & 13 & 103.45 & 104.19 & 102.29 & $1.60 \mathrm{E}-04$ & $1.37 \mathrm{E}-04$ & $1.32 \mathrm{E}-04$ \\
\hline 4 & 15 & 65 & 6.29 & 4.66 & $3.60 \mathrm{E}-04$ & $3.70 \mathrm{E}-04$ & $3.97 \mathrm{E}-04$ \\
\hline 4 & 41 & 1.89 & 1.87 & 11.89 & $\mathrm{E}-02$ & $6.87 \mathrm{E}-02$ & $6.92 \mathrm{E}-02$ \\
\hline 4 & 51 & .75 & 11.73 & 11.75 & $2.30 \mathrm{E}-02$ & $1.92 \mathrm{E}-02$ & $1.89 \mathrm{E}-02$ \\
\hline 4 & 52 & 1.75 & 11.73 & 11.75 & $3.80 \mathrm{E}-02$ & $3.68 \mathrm{E}-02$ & $3.68 \mathrm{E}-02$ \\
\hline 4 & 53 & 11.75 & 11.72 & 11.74 & $2.90 \mathrm{E}-02$ & $2.78 \mathrm{E}-02$ & $2.79 \mathrm{E}-02$ \\
\hline 4 & 146 & 9.07 & 9.05 & 9.06 & $7.70 \mathrm{E}-03$ & $7.39 \mathrm{E}-03$ & $7.23 \mathrm{E}-03$ \\
\hline 5 & 11 & 134.65 & 135.38 & 133.53 & $9.46 \mathrm{E}-02$ & $9.11 \mathrm{E}-02$ & $9.27 \mathrm{E}-02$ \\
\hline 5 & 12 & 113.23 & 113.91 & 111.81 & $6.30 \mathrm{E}-04$ & $5.83 \mathrm{E}-04$ & $7.01 \mathrm{E}-04$ \\
\hline 5 & 13 & 109.53 & 110.21 & 108.18 & $7.40 \mathrm{E}-03$ & $7.15 \mathrm{E}-03$ & $7.42 \mathrm{E}-03$ \\
\hline 5 & 15 & 89.73 & 90.38 & 88.66 & $2.10 \mathrm{E}-04$ & $1.94 \mathrm{E}-04$ & $2.04 \mathrm{E}-04$ \\
\hline 5 & 40 & 11.98 & 11.96 & 11.98 & $4.00 \mathrm{E}-02$ & $3.97 \mathrm{E}-02$ & $3.87 \mathrm{E}-02$ \\
\hline 5 & 50 & 1.84 & 11.82 & 11.84 & $1.50 \mathrm{E}-01$ & $1.57 \mathrm{E}-01$ & $1.59 \mathrm{E}-01$ \\
\hline 5 & 51 & 1.84 & 11.81 & 11.83 & $8.30 \mathrm{E}-02$ & $8.35 \mathrm{E}-02$ & $8.37 \mathrm{E}-02$ \\
\hline 5 & 52 & 11.82 & 11.80 & 11.82 & $2.60 \mathrm{E}-02$ & $2.64 \mathrm{E}-02$ & $2.65 \mathrm{E}-02$ \\
\hline 5 & 168 & 9.01 & 9.00 & 9.01 & $1.60 \mathrm{E}-02$ & $1.87 \mathrm{E}-02$ & $1.79 \mathrm{E}-02$ \\
\hline 5 & 169 & 9.01 & 9.00 & 9.01 & $1.10 \mathrm{E}-02$ & $1.08 \mathrm{E}-02$ & $1.01 \mathrm{E}-02$ \\
\hline 6 & 11 & 192.61 & 3.58 & 92.81 & $1.80 \mathrm{E}-03$ & $1.81 \mathrm{E}-03$ & $2.03 \mathrm{E}-03$ \\
\hline 6 & 12 & 151.54 & 152.49 & 150.57 & $5.20 \mathrm{E}-02$ & $4.99 \mathrm{E}-02$ & $5.04 \mathrm{E}-02$ \\
\hline 6 & 13 & 144.85 & 145.92 & 144.06 & $3.19 \mathrm{E}-02$ & $2.99 \mathrm{E}-02$ & $3.03 \mathrm{E}-02$ \\
\hline 6 & 14 & 120.03 & 120.80 & 118.92 & $5.05 \mathrm{E}-02$ & $4.73 \mathrm{E}-02$ & $4.77 \mathrm{E}-02$ \\
\hline 6 & 15 & 112.21 & 113.08 & 111.40 & $1.40 \mathrm{E}-02$ & $1.33 \mathrm{E}-02$ & $1.35 \mathrm{E}-02$ \\
\hline 6 & 49 & 12.19 & 12.18 & 12.21 & $5.30 \mathrm{E}-02$ & $4.18 \mathrm{E}-02$ & $4.02 \mathrm{E}-02$ \\
\hline 6 & 54 & 12.05 & 12.07 & 12.10 & $3.20 \mathrm{E}-02$ & $3.55 \mathrm{E}-02$ & $3.59 \mathrm{E}-02$ \\
\hline 6 & 67 & 11.75 & 11.73 & 11.75 & $7.70 \mathrm{E}-02$ & $9.04 \mathrm{E}-02$ & $9.12 \mathrm{E}-02$ \\
\hline 6 & 156 & 9.24 & 9.24 & 9.26 & $1.20 \mathrm{E}-02$ & $1.32 \mathrm{E}-02$ & $1.23 \mathrm{E}-02$ \\
\hline 6 & 194 & 8.96 & 8.95 & 8.96 & $8.10 \mathrm{E}-03$ & $9.98 \mathrm{E}-03$ & $9.50 \mathrm{E}-03$ \\
\hline
\end{tabular}


Table 4. continued.

\begin{tabular}{|c|c|c|c|c|c|c|c|}
\hline$k$ & $i$ & $\lambda^{\text {NIST }}(\AA)$ & $\lambda^{\text {CITRO }}(\AA)$ & $\lambda^{\text {GRASP }}(\AA)$ & $S^{\text {NIST }}$ & $S^{\text {CITRO }}$ & $S^{\text {GRASP }}$ \\
\hline 7 & 12 & 157.03 & 157.98 & 155.78 & $3.80 \mathrm{E}-02$ & $3.56 \mathrm{E}-02$ & $3.64 \mathrm{E}-02$ \\
\hline 7 & 13 & 149.87 & 150.94 & 148.82 & $1.28 \mathrm{E}-01$ & $1.22 \mathrm{E}-01$ & $1.23 \mathrm{E}-01$ \\
\hline 7 & 15 & 115.19 & 116.07 & 114.22 & $4.31 \mathrm{E}-02$ & $4.09 \mathrm{E}-02$ & $4.11 \mathrm{E}-02$ \\
\hline 7 & 54 & 12.10 & 12.10 & 12.13 & $4.10 \mathrm{E}-02$ & $3.68 \mathrm{E}-02$ & $3.64 \mathrm{E}-02$ \\
\hline 7 & 56 & 12.05 & 12.03 & 12.05 & $1.70 \mathrm{E}-01$ & $1.55 \mathrm{E}-01$ & $1.53 \mathrm{E}-01$ \\
\hline 7 & 66 & 11.79 & 11.77 & 11.78 & $7.80 \mathrm{E}-02$ & $8.88 \mathrm{E}-02$ & $9.07 \mathrm{E}-02$ \\
\hline 7 & 172 & 9.22 & 9.19 & 9.21 & $6.30 \mathrm{E}-03$ & $6.65 \mathrm{E}-03$ & $6.32 \mathrm{E}-03$ \\
\hline 7 & 174 & 8.98 & 9.17 & 9.19 & $7.10 \mathrm{E}-03$ & $2.80 \mathrm{E}-02$ & $2.62 \mathrm{E}-02$ \\
\hline 7 & 195 & 9.18 & 8.97 & 8.98 & $2.50 \mathrm{E}-02$ & $9.27 \mathrm{E}-03$ & $8.88 \mathrm{E}-03$ \\
\hline 8 & 11 & 248.62 & 250.99 & 251.09 & $3.60 \mathrm{E}-03$ & $3.56 \mathrm{E}-03$ & $3.97 \mathrm{E}-03$ \\
\hline 8 & 12 & 184.18 & 186.01 & 183.90 & $8.20 \mathrm{E}-02$ & $7.74 \mathrm{E}-02$ & $7.83 \mathrm{E}-02$ \\
\hline 8 & 14 & 139.64 & 140.92 & 138.79 & $7.00 \mathrm{E}-03$ & $6.93 \mathrm{E}-03$ & $6.93 \mathrm{E}-03$ \\
\hline 8 & 15 & 129.17 & 130.52 & 128.65 & $1.59 \mathrm{E}-02$ & $1.52 \mathrm{E}-02$ & $1.52 \mathrm{E}-02$ \\
\hline 8 & 55 & 12.01 & 12.19 & 12.22 & $2.50 \mathrm{E}-02$ & $2.54 \mathrm{E}-02$ & $2.53 \mathrm{E}-02$ \\
\hline 8 & 59 & 12.33 & 12.14 & 12.17 & $2.80 \mathrm{E}-02$ & $4.60 \mathrm{E}-03$ & $4.59 \mathrm{E}-03$ \\
\hline 8 & 69 & 12.08 & 11.88 & 11.90 & $3.50 \mathrm{E}-02$ & $2.65 \mathrm{E}-02$ & $2.63 \mathrm{E}-02$ \\
\hline 8 & 74 & 12.03 & 11.83 & 11.85 & $2.40 \mathrm{E}-02$ & $3.77 \mathrm{E}-03$ & $3.80 \mathrm{E}-03$ \\
\hline 9 & 11 & 360.39 & 362.21 & 365.09 & $1.10 \mathrm{E}-03$ & $9.46 \mathrm{E}-04$ & $1.02 \mathrm{E}-03$ \\
\hline 9 & 12 & 239.12 & 240.81 & 238.43 & $2.30 \mathrm{E}-02$ & $1.94 \mathrm{E}-02$ & $1.93 \mathrm{E}-02$ \\
\hline 9 & 14 & 169.08 & 170.27 & 167.74 & $5.70 \mathrm{E}-02$ & $5.18 \mathrm{E}-02$ & $5.25 \mathrm{E}-02$ \\
\hline 9 & 15 & 153.96 & 155.32 & 153.15 & $1.25 \mathrm{E}-02$ & $1.26 \mathrm{E}-02$ & $1.30 \mathrm{E}-02$ \\
\hline 9 & 55 & 12.19 & 12.38 & 12.41 & $2.60 \mathrm{E}-02$ & $1.68 \mathrm{E}-02$ & $1.67 \mathrm{E}-02$ \\
\hline 9 & 59 & 12.52 & 12.32 & 12.36 & $2.90 \mathrm{E}-02$ & $2.51 \mathrm{E}-02$ & $2.49 \mathrm{E}-02$ \\
\hline 9 & 69 & 12.26 & 12.06 & 12.08 & $3.60 \mathrm{E}-02$ & $3.34 \mathrm{E}-02$ & $3.28 \mathrm{E}-02$ \\
\hline 10 & 11 & 379.64 & 381.36 & 385.38 & $3.30 \mathrm{E}-03$ & $3.05 \mathrm{E}-03$ & $3.24 \mathrm{E}-03$ \\
\hline 10 & 12 & 247.45 & 249.13 & 246.92 & $1.30 \mathrm{E}-03$ & $1.53 \mathrm{E}-03$ & $1.56 \mathrm{E}-03$ \\
\hline 10 & 13 & 230.10 & 232.06 & 229.89 & $1.22 \mathrm{E}-01$ & $1.15 \mathrm{E}-01$ & $1.16 \mathrm{E}-01$ \\
\hline 10 & 14 & 173.21 & 174.39 & 171.89 & $1.10 \mathrm{E}-02$ & $1.07 \mathrm{E}-02$ & $1.07 \mathrm{E}-02$ \\
\hline 10 & 15 & 157.37 & 158.74 & 156.61 & $1.50 \mathrm{E}-01$ & $1.43 \mathrm{E}-01$ & $1.45 \mathrm{E}-01$ \\
\hline 10 & 55 & 12.21 & 12.40 & 12.43 & $3.50 \mathrm{E}-02$ & $8.48 \mathrm{E}-03$ & $8.45 \mathrm{E}-03$ \\
\hline 10 & 71 & 12.08 & 12.06 & 12.08 & $1.30 \mathrm{E}-01$ & $1.20 \mathrm{E}-01$ & $1.19 \mathrm{E}-01$ \\
\hline 10 & 196 & 9.16 & 9.15 & 9.17 & $1.60 \mathrm{E}-02$ & $1.72 \mathrm{E}-02$ & $1.64 \mathrm{E}-02$ \\
\hline
\end{tabular}


Table 5. The five major spontaneous radiative transition probabilities $A^{r}$ from each level of Fe XXII and the sum of all radiative probabilities $\sum A^{r}$ from the corresponding level. Electric dipole, quadrupole and octupole as well as magnetic dipole and quadrupole transitions are included. Arrow marks the final level to which radiative transition happens from the level.

\begin{tabular}{|c|c|c|c|c|c|c|c|c|c|c|c|}
\hline Level & $A^{r}\left(\mathrm{~s}^{-1}\right)$ & $\begin{array}{l}\text { final } \\
\text { level }\end{array}$ & $A^{r}\left(\mathrm{~s}^{-1}\right)$ & $\begin{array}{l}\text { final } \\
\text { level }\end{array}$ & $A^{r}\left(\mathrm{~s}^{-1}\right)$ & $\begin{array}{l}\text { final } \\
\text { level }\end{array}$ & $A^{r}\left(\mathrm{~s}^{-1}\right)$ & $\begin{array}{l}\text { final } \\
\text { level }\end{array}$ & $A^{r}\left(\mathrm{~s}^{-1}\right)$ & $\begin{array}{l}\text { final } \\
\text { level }\end{array}$ & $\sum A^{r}\left(\mathrm{~s}^{-1}\right)$ \\
\hline$\overline{2}$ & $1.47 \mathrm{E}+04$ & $\rightarrow 1$ & & & & & & & & & $1.47 \mathrm{E}+04$ \\
\hline 3 & $7.84 \mathrm{E}+07$ & $\rightarrow 1$ & $1.30 \mathrm{E}+07$ & $\rightarrow 2$ & & & & & & & $9.14 \mathrm{E}+07$ \\
\hline 4 & $7.93 \mathrm{E}+06$ & $\rightarrow 2$ & $1.89 \mathrm{E}+06$ & $\rightarrow 1$ & $3.60 \mathrm{E}+03$ & $\rightarrow 3$ & & & & & $9.82 \mathrm{E}+06$ \\
\hline 5 & $6.78 \mathrm{E}+07$ & $\rightarrow 2$ & $2.36 \mathrm{E}+03$ & $\rightarrow 4$ & $6.81 \mathrm{E}+00$ & $\rightarrow 1$ & $6.27 \mathrm{E}-01$ & $\rightarrow 3$ & & & $6.78 \mathrm{E}+07$ \\
\hline 6 & $1.09 \mathrm{E}+10$ & $\rightarrow 1$ & $3.25 \mathrm{E}+07$ & $\rightarrow 2$ & $7.86 \mathrm{E}+03$ & $\rightarrow 4$ & $4.95 \mathrm{E}+03$ & $\rightarrow 5$ & $3.50 \mathrm{E}+03$ & $\rightarrow 3$ & $1.10 \mathrm{E}+10$ \\
\hline 7 & $6.06 \mathrm{E}+09$ & $\rightarrow 2$ & $3.10 \mathrm{E}+04$ & $\rightarrow 5$ & $1.42 \mathrm{E}+04$ & $\rightarrow 4$ & $1.10 \mathrm{E}+02$ & $\rightarrow 6$ & $3.93 \mathrm{E}+01$ & $\rightarrow 1$ & $6.06 \mathrm{E}+09$ \\
\hline 8 & $3.92 \mathrm{E}+10$ & $\rightarrow 1$ & $6.88 \mathrm{E}+07$ & $\rightarrow 2$ & $5.52 \mathrm{E}+04$ & $\rightarrow 4$ & $5.48 \mathrm{E}+04$ & $\rightarrow 3$ & $7.23 \mathrm{E}+02$ & $\rightarrow 6$ & $3.93 \mathrm{E}+10$ \\
\hline 9 & $3.47 \mathrm{E}+10$ & $\rightarrow 2$ & $2.22 \mathrm{E}+09$ & $\rightarrow 1$ & $1.01 \mathrm{E}+05$ & $\rightarrow 4$ & $1.69 \mathrm{E}+04$ & $\rightarrow 8$ & $5.53 \mathrm{E}+03$ & $\rightarrow 6$ & $3.69 \mathrm{E}+10$ \\
\hline 10 & $4.52 \mathrm{E}+10$ & $\rightarrow 2$ & $6.36 \mathrm{E}+09$ & $\rightarrow 1$ & $2.01 \mathrm{E}+04$ & $\rightarrow 6$ & $1.67 \mathrm{E}+04$ & $\rightarrow 4$ & $1.43 \mathrm{E}+04$ & $\rightarrow 8$ & $5.16 \mathrm{E}+10$ \\
\hline 11 & $1.94 \mathrm{E}+10$ & $\rightarrow 5$ & $1.51 \mathrm{E}+10$ & $\rightarrow 4$ & $1.02 \mathrm{E}+10$ & $\rightarrow 3$ & $1.28 \mathrm{E}+08$ & $\rightarrow 6$ & $1.14 \mathrm{E}+08$ & $\rightarrow 8$ & $4.49 \mathrm{E}+10$ \\
\hline 12 & $7.41 \mathrm{E}+09$ & $\rightarrow 6$ & $6.30 \mathrm{E}+09$ & $\rightarrow 8$ & $4.77 \mathrm{E}+09$ & $\rightarrow 7$ & $2.20 \mathrm{E}+09$ & $\rightarrow 4$ & $7.26 \mathrm{E}+08$ & $\rightarrow 9$ & $2.17 \mathrm{E}+10$ \\
\hline 13 & $1.25 \mathrm{E}+10$ & $\rightarrow 7$ & $3.38 \mathrm{E}+09$ & $\rightarrow 6$ & $3.18 \mathrm{E}+09$ & $\rightarrow 10$ & $1.91 \mathrm{E}+09$ & $\rightarrow 5$ & $4.34 \mathrm{E}+07$ & $\rightarrow 4$ & $2.10 \mathrm{E}+10$ \\
\hline 14 & $2.85 \mathrm{E}+10$ & $\rightarrow 6$ & $1.11 \mathrm{E}+10$ & $\rightarrow 9$ & $2.62 \mathrm{E}+09$ & $\rightarrow 8$ & $2.13 \mathrm{E}+09$ & $\rightarrow 10$ & $2.17 \mathrm{E}+08$ & $\rightarrow 3$ & $4.46 \mathrm{E}+10$ \\
\hline 15 & $1.89 \mathrm{E}+10$ & $\rightarrow 10$ & $1.39 \mathrm{E}+10$ & $\rightarrow 7$ & $4.87 \mathrm{E}+09$ & $\rightarrow 6$ & $3.62 \mathrm{E}+09$ & $\rightarrow 8$ & $1.78 \mathrm{E}+09$ & $\rightarrow 9$ & $4.35 E+10$ \\
\hline 16 & $1.90 \mathrm{E}+12$ & $\rightarrow 2$ & $8.94 \mathrm{E}+11$ & $\rightarrow 1$ & $1.18 \mathrm{E}+08$ & $\rightarrow 15$ & $9.19 \mathrm{E}+07$ & $\rightarrow 11$ & $5.64 \mathrm{E}+07$ & $\rightarrow 12$ & $2.79 E+12$ \\
\hline 17 & $1.92 \mathrm{E}+11$ & $\rightarrow 6$ & $3.71 \mathrm{E}+10$ & $\rightarrow 9$ & $3.90 \mathrm{E}+09$ & $\rightarrow 2$ & $3.74 \mathrm{E}+09$ & $\rightarrow 4$ & $1.00 \mathrm{E}+09$ & $\rightarrow 16$ & $2.38 \mathrm{E}+11$ \\
\hline 18 & $1.56 \mathrm{E}+11$ & $\rightarrow 7$ & $2.70 \mathrm{E}+10$ & $\rightarrow 8$ & $2.56 \mathrm{E}+10$ & $\rightarrow 10$ & $2.30 \mathrm{E}+10$ & $\rightarrow 5$ & $1.06 \mathrm{E}+10$ & $\rightarrow 6$ & $2.52 \mathrm{E}+11$ \\
\hline 19 & $2.48 \mathrm{E}+12$ & $\rightarrow 4$ & $5.38 \mathrm{E}+11$ & $\rightarrow 3$ & $2.84 \mathrm{E}+10$ & $\rightarrow 6$ & $3.85 \mathrm{E}+09$ & $\rightarrow 9$ & $6.30 \mathrm{E}+08$ & $\rightarrow 8$ & $3.05 \mathrm{E}+12$ \\
\hline 20 & $1.64 \mathrm{E}+13$ & $\rightarrow 1$ & $3.15 \mathrm{E}+12$ & $\rightarrow 2$ & $1.21 \mathrm{E}+09$ & $\rightarrow 12$ & $8.63 \mathrm{E}+08$ & $\rightarrow 17$ & $1.13 \mathrm{E}+08$ & $\rightarrow 8$ & $1.95 \mathrm{E}+13$ \\
\hline 21 & $1.88 \mathrm{E}+13$ & $\rightarrow 2$ & $1.10 \mathrm{E}+09$ & $\rightarrow 13$ & $7.29 \mathrm{E}+08$ & $\rightarrow 18$ & $2.19 \mathrm{E}+08$ & $\rightarrow 15$ & $1.38 \mathrm{E}+08$ & $\rightarrow 11$ & $1.88 \mathrm{E}+13$ \\
\hline 22 & $1.45 E+12$ & $\rightarrow 5$ & $1.33 \mathrm{E}+12$ & $\rightarrow 3$ & $3.47 \mathrm{E}+11$ & $\rightarrow 4$ & $3.52 \mathrm{E}+10$ & $\rightarrow 6$ & $2.41 \mathrm{E}+10$ & $\rightarrow 7$ & $3.19 \mathrm{E}+12$ \\
\hline 23 & $1.54 \mathrm{E}+12$ & $\rightarrow 6$ & $6.16 \mathrm{E}+11$ & $\rightarrow 8$ & $5.13 \mathrm{E}+10$ & $\rightarrow 9$ & $2.54 \mathrm{E}+10$ & $\rightarrow 4$ & $4.83 \mathrm{E}+09$ & $\rightarrow 10$ & $2.24 \mathrm{E}+12$ \\
\hline 24 & $2.20 \mathrm{E}+12$ & $\rightarrow 5$ & $E+12$ & $\rightarrow 4$ & $1.06 \mathrm{E}+11$ & $\rightarrow 7$ & $3.01 \mathrm{E}+09$ & $\rightarrow 6$ & $2.66 \mathrm{E}+09$ & $\rightarrow 10$ & $3.42 \mathrm{E}+12$ \\
\hline 25 & $1.53 \mathrm{E}+12$ & $\rightarrow 1$ & $2.18 \mathrm{E}+11$ & $\rightarrow 2$ & $1.46 \mathrm{E}+10$ & $\rightarrow 12$ & $2.95 \mathrm{E}+09$ & $\rightarrow 4$ & $2.25 \mathrm{E}+09$ & $\rightarrow 14$ & $1.77 \mathrm{E}+12$ \\
\hline 26 & $7.38 \mathrm{E}+11$ & $\rightarrow 2$ & $7.17 \mathrm{E}+11$ & $\rightarrow 1$ & $1.01 \mathrm{E}+10$ & $\rightarrow 13$ & $2.06 \mathrm{E}+09$ & $\rightarrow 14$ & $1.22 \mathrm{E}+09$ & $\rightarrow 4$ & $1.47 \mathrm{E}+12$ \\
\hline 27 & $1.89 \mathrm{E}+12$ & $\rightarrow 7$ & $5.21 \mathrm{E}+11$ & $\rightarrow 9$ & +11 & $\rightarrow 8$ & $1.47 \mathrm{E}+11$ & $\rightarrow 6$ & $E+11$ & $\rightarrow 10$ & $E+12$ \\
\hline 28 & $2.35 \mathrm{E}+12$ & $\rightarrow 1$ & $9.90 \mathrm{E}+11$ & $\rightarrow 2$ & $3.09 \mathrm{E}+10$ & $\rightarrow 13$ & $3.44 \mathrm{E}+09$ & $\rightarrow 11$ & $3.26 \mathrm{E}+09$ & $\rightarrow 14$ & $3.38 \mathrm{E}+12$ \\
\hline 29 & $6.49 \mathrm{E}+12$ & $\rightarrow 1$ & $6.12 \mathrm{E}+11$ & $\rightarrow 2$ & $1.16 \mathrm{E}+11$ & $\rightarrow 12$ & $5.06 \mathrm{E}+09$ & $\rightarrow 11$ & $5.03 E+09$ & $\rightarrow 14$ & $7.23 \mathrm{E}+12$ \\
\hline 30 & $4.15 \mathrm{E}+10$ & $\rightarrow 2$ & $2.93 \mathrm{E}+09$ & $\rightarrow 11$ & $2.02 \mathrm{E}+09$ & $\rightarrow 3$ & $1.64 \mathrm{E}+09$ & $\rightarrow 5$ & $1.48 \mathrm{E}+09$ & $\rightarrow 22$ & $5.09 \mathrm{E}+10$ \\
\hline 31 & $2.52 \mathrm{E}+11$ & $\rightarrow 1$ & $1.52 \mathrm{E}+11$ & $\rightarrow 2$ & $2.53 \mathrm{E}+10$ & $\rightarrow 11$ & $3.31 \mathrm{E}+09$ & $\rightarrow 14$ & $2.91 \mathrm{E}+09$ & $\rightarrow 15$ & $4.42 \mathrm{E}+11$ \\
\hline 32 & $4.91 \mathrm{E}+12$ & $\rightarrow 1$ & $5.06 \mathrm{E}+10$ & $\rightarrow 2$ & $3.85 \mathrm{E}+10$ & $\rightarrow 13$ & $1.21 \mathrm{E}+10$ & $\rightarrow 12$ & $8.63 E+09$ & $\rightarrow 14$ & $5.03 \mathrm{E}+12$ \\
\hline 33 & $2.27 \mathrm{E}+09$ & $\rightarrow 5$ & $1.62 \mathrm{E}+09$ & $\rightarrow 4$ & $1.43 \mathrm{E}+09$ & $\rightarrow 24$ & $1.18 \mathrm{E}+08$ & $\rightarrow 7$ & $1.73 \mathrm{E}+07$ & $\rightarrow 13$ & $5.47 \mathrm{E}+09$ \\
\hline 34 & $3.26 \mathrm{E}+12$ & $\rightarrow 2$ & $1.04 \mathrm{E}+12$ & $\rightarrow 1$ & $4.01 \mathrm{E}+10$ & $\rightarrow 12$ & $7.58 \mathrm{E}+09$ & $\rightarrow 13$ & $7.20 \mathrm{E}+09$ & $\rightarrow 14$ & $4.36 \mathrm{E}+12$ \\
\hline 35 & $6.48 \mathrm{E}+11$ & $\rightarrow 4$ & $6.38 \mathrm{E}+11$ & $\rightarrow 3$ & $4.97 \mathrm{E}+11$ & $\rightarrow 6$ & $4.72 \mathrm{E}+10$ & $\rightarrow 5$ & $2.27 \mathrm{E}+10$ & $\rightarrow 7$ & $1.87 \mathrm{E}+12$ \\
\hline 36 & $2.56 \mathrm{E}+12$ & $\rightarrow 2$ & $7.38 \mathrm{E}+11$ & $\rightarrow 1$ & $2.19 E+10$ & $\rightarrow 12$ & $1.23 \mathrm{E}+10$ & $\rightarrow 11$ & $6.51 \mathrm{E}+09$ & $\rightarrow 13$ & $3.35 E+12$ \\
\hline 37 & $2.13 \mathrm{E}+12$ & $\rightarrow 2$ & $1.43 \mathrm{E}+10$ & $\rightarrow 13$ & $1.22 \mathrm{E}+10$ & $\rightarrow 11$ & $9.91 \mathrm{E}+09$ & $\rightarrow 15$ & $1.61 \mathrm{E}+09$ & $\rightarrow 24$ & $2.17 \mathrm{E}+12$ \\
\hline 38 & +12 & $\rightarrow 4$ & +11 & $\rightarrow 5$ & 11 & $\rightarrow 7$ & 11 & $\rightarrow 6$ & -09 & $\rightarrow 10$ & $3.81 \mathrm{E}+12$ \\
\hline 39 & $7.32 \mathrm{E}+12$ & $\rightarrow 2$ & $6.68 \mathrm{E}+10$ & $\rightarrow 13$ & $2.08 \mathrm{E}+10$ & $\rightarrow 15$ & $1.32 \mathrm{E}+10$ & $\rightarrow 11$ & $1.09 \mathrm{E}+09$ & $\rightarrow 18$ & $7.43 \mathrm{E}+12$ \\
\hline 40 & $5.84 \mathrm{E}+12$ & $\rightarrow 5$ & $2.49 \mathrm{E}+11$ & $\rightarrow 7$ & $4.96 \mathrm{E}+08$ & $\rightarrow 30$ & $2.91 \mathrm{E}+07$ & $\rightarrow 2$ & $7.05 \mathrm{E}+06$ & $\rightarrow 21$ & $6.09 \mathrm{E}+12$ \\
\hline 41 & $1.38 \mathrm{E}+13$ & $\rightarrow 4$ & $1.23 \mathrm{E}+12$ & $\rightarrow 7$ & $1.13 \mathrm{E}+12$ & $\rightarrow 5$ & $5.26 \mathrm{E}+11$ & $\rightarrow 6$ & $1.68 \mathrm{E}+11$ & $\rightarrow 10$ & +13 \\
\hline 42 & $1.66 \mathrm{E}+13$ & $\rightarrow 3$ & $5.23 \mathrm{E}+12$ & $\rightarrow 4$ & $1.11 \mathrm{E}+12$ & $\rightarrow 6$ & $2.93 \mathrm{E}+11$ & $\rightarrow 8$ & $2.27 \mathrm{E}+11$ & $\rightarrow 5$ & $2.36 \mathrm{E}+13$ \\
\hline 43 & $2.66 \mathrm{E}+13$ & $\rightarrow 3$ & $1.27 \mathrm{E}+12$ & $\rightarrow 4$ & $2.62 \mathrm{E}+11$ & $\rightarrow 6$ & $2.12 \mathrm{E}+11$ & $\rightarrow 9$ & $5.16 \mathrm{E}+10$ & $\rightarrow 10$ & $2.84 \mathrm{E}+13$ \\
\hline 44 & $7.66 \mathrm{E}+12$ & $\rightarrow 2$ & $1.53 \mathrm{E}+12$ & $\rightarrow 1$ & $1.82 \mathrm{E}+11$ & $\rightarrow 15$ & $9.42 \mathrm{E}+10$ & $\rightarrow 14$ & $2.07 \mathrm{E}+10$ & $\rightarrow 11$ & $9.51 \mathrm{E}+12$ \\
\hline 45 & $1.71 \mathrm{E}+12$ & $\rightarrow 9$ & $1.23 \mathrm{E}+12$ & $\rightarrow 6$ & $9.69 \mathrm{E}+11$ & $\rightarrow 10$ & $9.34 \mathrm{E}+11$ & $\rightarrow 3$ & $4.22 \mathrm{E}+10$ & $\rightarrow 8$ & $4.92 \mathrm{E}+12$ \\
\hline 46 & $2.25 E+12$ & $\rightarrow 8$ & $1.15 \mathrm{E}+12$ & $\rightarrow 10$ & $9.86 \mathrm{E}+11$ & $\rightarrow 6$ & $7.98 \mathrm{E}+11$ & $\rightarrow 7$ & $5.62 \mathrm{E}+11$ & $\rightarrow 3$ & $6.08 \mathrm{E}+12$ \\
\hline 47 & $3.74 \mathrm{E}+12$ & $\rightarrow 6$ & $3.21 \mathrm{E}+12$ & $\rightarrow 8$ & $1.28 \mathrm{E}+12$ & $\rightarrow 3$ & $5.75 \mathrm{E}+11$ & $\rightarrow 10$ & $5.64 \mathrm{E}+11$ & $\rightarrow 7$ & $1.02 \mathrm{E}+13$ \\
\hline 48 & $4.56 \mathrm{E}+08$ & $\rightarrow 33$ & $1.24 \mathrm{E}+07$ & $\rightarrow 7$ & $1.67 \mathrm{E}+06$ & $\rightarrow 5$ & $9.33 \mathrm{E}+05$ & $\rightarrow 4$ & $7.28 \mathrm{E}+03$ & $\rightarrow 24$ & $4.70 \mathrm{E}+08$ \\
\hline 49 & $7.75 \mathrm{E}+12$ & $\rightarrow 6$ & $4.71 \mathrm{E}+12$ & $\rightarrow 4$ & $1.23 \mathrm{E}+12$ & $\rightarrow 7$ & $6.54 \mathrm{E}+11$ & $\rightarrow 10$ & $2.64 \mathrm{E}+11$ & $\rightarrow 5$ & $1.46 \mathrm{E}+13$ \\
\hline 50 & $2.40 \mathrm{E}+13$ & $\rightarrow 5$ & $2.21 \mathrm{E}+11$ & $\rightarrow 7$ & $2.59 \mathrm{E}+08$ & $\rightarrow 37$ & $2.34 \mathrm{E}+08$ & $\rightarrow 33$ & $7.73 \mathrm{E}+07$ & $\rightarrow 2$ & $2.42 \mathrm{E}+13$ \\
\hline 51 & $1.70 \mathrm{E}+13$ & $\rightarrow 5$ & $4.00 \mathrm{E}+12$ & $\rightarrow 4$ & $1.17 \mathrm{E}+12$ & $\rightarrow 6$ & $3.20 \mathrm{E}+11$ & $\rightarrow 7$ & $1.44 \mathrm{E}+10$ & $\rightarrow 10$ & $2.25 \mathrm{E}+13$ \\
\hline 52 & $1.15 \mathrm{E}+13$ & $\rightarrow 4$ & $8.10 \mathrm{E}+12$ & $\rightarrow 5$ & $3.09 \mathrm{E}+11$ & $\rightarrow 7$ & $1.49 \mathrm{E}+11$ & $\rightarrow 8$ & $1.11 \mathrm{E}+11$ & $\rightarrow 3$ & $2.03 \mathrm{E}+13$ \\
\hline 53 & $1.74 \mathrm{E}+13$ & $\rightarrow 4$ & $5.08 \mathrm{E}+10$ & $\rightarrow 3$ & $3.72 \mathrm{E}+10$ & $\rightarrow 10$ & $3.19 \mathrm{E}+10$ & $\rightarrow 6$ & $1.57 \mathrm{E}+10$ & $\rightarrow 9$ & $1.75 E+13$ \\
\hline 54 & $6.96 \mathrm{E}+12$ & $\rightarrow 7$ & $6.76 \mathrm{E}+12$ & $\rightarrow 6$ & $1.02 \mathrm{E}+12$ & $\rightarrow 10$ & $4.20 \mathrm{E}+11$ & $\rightarrow 5$ & $1.32 \mathrm{E}+11$ & $\rightarrow 4$ & $1.53 \mathrm{E}+13$ \\
\hline 55 & $7.05 \mathrm{E}+12$ & $\rightarrow 8$ & $4.44 \mathrm{E}+12$ & $\rightarrow 9$ & $2.24 \mathrm{E}+12$ & $\rightarrow 10$ & $9.64 \mathrm{E}+11$ & $\rightarrow 7$ & $3.20 \mathrm{E}+11$ & $\rightarrow 6$ & $1.53 \mathrm{E}+13$ \\
\hline 56 & $2.25 \mathrm{E}+13$ & $\rightarrow 7$ & $1.23 \mathrm{E}+11$ & $\rightarrow 5$ & $1.50 \mathrm{E}+10$ & $\rightarrow 2$ & $1.10 \mathrm{E}+09$ & $\rightarrow 39$ & $4.12 \mathrm{E}+08$ & $\rightarrow 37$ & $2.26 \mathrm{E}+13$ \\
\hline 57 & $1.99 \mathrm{E}+12$ & $\rightarrow 2$ & $7.91 \mathrm{E}+11$ & $\rightarrow 1$ & $5.31 \mathrm{E}+11$ & $\rightarrow 12$ & $1.46 \mathrm{E}+11$ & $\rightarrow 14$ & $7.69 \mathrm{E}+10$ & $\rightarrow 11$ & $3.56 \mathrm{E}+12$ \\
\hline 58 & $1.74 \mathrm{E}+12$ & $\rightarrow 2$ & $1.44 \mathrm{E}+11$ & $\rightarrow 13$ & $2.13 \mathrm{E}+10$ & $\rightarrow 14$ & $1.82 \mathrm{E}+10$ & $\rightarrow 15$ & $1.78 \mathrm{E}+10$ & $\rightarrow 17$ & $1.97 \mathrm{E}+12$ \\
\hline 59 & $1.35 \mathrm{E}+13$ & $\rightarrow 9$ & $2.59 \mathrm{E}+12$ & $\rightarrow 8$ & $9.03 E+11$ & $\rightarrow 10$ & $2.79 E+11$ & $\rightarrow 6$ & $1.56 \mathrm{E}+11$ & $\rightarrow 3$ & $1.74 \mathrm{E}+13$ \\
\hline 60 & $1.08 \mathrm{E}+12$ & $\rightarrow 2$ & $1.63 \mathrm{E}+10$ & $\rightarrow 18$ & $7.82 \mathrm{E}+09$ & $\rightarrow 15$ & $6.10 \mathrm{E}+09$ & $\rightarrow 12$ & $1.91 \mathrm{E}+09$ & $\rightarrow 11$ & $1.12 \mathrm{E}+12$ \\
\hline
\end{tabular}


Table 5. continued.

\begin{tabular}{|c|c|c|c|c|c|c|c|c|c|c|c|}
\hline Level & $A^{r}\left(\mathrm{~s}^{-1}\right)$ & $\begin{array}{l}\text { final } \\
\text { level }\end{array}$ & $A^{r}\left(\mathrm{~s}^{-1}\right)$ & $\begin{array}{l}\text { final } \\
\text { level }\end{array}$ & $A^{r}\left(\mathrm{~s}^{-1}\right)$ & $\begin{array}{l}\text { final } \\
\text { level }\end{array}$ & $A^{r}\left(\mathrm{~s}^{-1}\right)$ & $\begin{array}{l}\text { final } \\
\text { level }\end{array}$ & $A^{r}\left(\mathrm{~s}^{-1}\right)$ & $\begin{array}{l}\text { final } \\
\text { level }\end{array}$ & $\sum A^{r}\left(\mathrm{~s}^{-1}\right)$ \\
\hline$\overline{61}$ & $1.53 \mathrm{E}+12$ & $\rightarrow 2$ & $55 \mathrm{E}+11$ & $\rightarrow 1$ & $3.78 \mathrm{E}+11$ & $\rightarrow 13$ & $1.20 \mathrm{E}+11$ & $\rightarrow 15$ & $3.04 \mathrm{E}+10$ & $\rightarrow 14$ & $2.57 \mathrm{E}+12$ \\
\hline 62 & $9.67 \mathrm{E}+11$ & $\rightarrow 11$ & $75 E+11$ & $\rightarrow 2$ & $.93 \mathrm{E}+10$ & $\rightarrow 12$ & $1.40 \mathrm{E}+10$ & $\rightarrow 14$ & $1.22 \mathrm{E}+10$ & $\rightarrow 1$ & $1.35 \mathrm{E}+12$ \\
\hline 63 & $6.34 \mathrm{E}+11$ & $\rightarrow 11$ & $4.71 \mathrm{E}+11$ & $\rightarrow 1$ & $1.26 \mathrm{E}+11$ & $\rightarrow 2$ & $1.97 \mathrm{E}+10$ & $\rightarrow 14$ & $1.86 \mathrm{E}+10$ & $\rightarrow 12$ & $1.30 \mathrm{E}+12$ \\
\hline 64 & $1.53 \mathrm{E}+12$ & $\rightarrow 11$ & $1.34 \mathrm{E}+11$ & $\rightarrow 12$ & $9.37 \mathrm{E}+10$ & $\rightarrow 2$ & $1.10 \mathrm{E}+10$ & $\rightarrow 13$ & $8.82 \mathrm{E}+09$ & $\rightarrow 19$ & $1.79 \mathrm{E}+12$ \\
\hline 65 & $1.74 \mathrm{E}+12$ & $\rightarrow 11$ & $3.24 \mathrm{E}+11$ & $\rightarrow 13$ & $8.22 \mathrm{E}+10$ & $\rightarrow 2$ & $9.14 \mathrm{E}+09$ & $\rightarrow 24$ & $6.66 \mathrm{E}+09$ & $\rightarrow 22$ & $2.17 \mathrm{E}+12$ \\
\hline 66 & $1.38 \mathrm{E}+13$ & $\rightarrow 7$ & $1.55 \mathrm{E}+11$ & $\rightarrow 5$ & $1.20 \mathrm{E}+10$ & $\rightarrow 21$ & $4.43 E+09$ & $\rightarrow 2$ & $5.04 \mathrm{E}+08$ & $\rightarrow 60$ & $1.39 \mathrm{E}+13$ \\
\hline 67 & $1.88 \mathrm{E}+13$ & $\rightarrow 6$ & $1.47 \mathrm{E}+12$ & $\rightarrow 7$ & $2.42 \mathrm{E}+10$ & $\rightarrow 5$ & $2.39 \mathrm{E}+10$ & $\rightarrow 10$ & $2.08 \mathrm{E}+10$ & $\rightarrow 4$ & $2.04 \mathrm{E}+13$ \\
\hline 68 & $2.41 \mathrm{E}+12$ & $\rightarrow 12$ & $7.73 \mathrm{E}+11$ & $\rightarrow 14$ & $4.86 \mathrm{E}+11$ & $\rightarrow 1$ & $2.86 \mathrm{E}+11$ & $\rightarrow 2$ & $1.01 \mathrm{E}+11$ & $\rightarrow 15$ & $4.09 \mathrm{E}+12$ \\
\hline 69 & $9.60 \mathrm{E}+12$ & $\rightarrow 9$ & $7.97 \mathrm{E}+12$ & $\rightarrow 8$ & $3.18 \mathrm{E}+12$ & $\rightarrow 6$ & $.08 \mathrm{E}+12$ & $\rightarrow 10$ & $.12 \mathrm{E}+11$ & $\rightarrow 7$ & $2.40 \mathrm{E}+13$ \\
\hline 70 & $4.32 \mathrm{E}+12$ & $\rightarrow 8$ & $2.10 \mathrm{E}+12$ & $\rightarrow 3$ & $3.33 \mathrm{E}+11$ & $\rightarrow 9$ & $5.44 \mathrm{E}+10$ & $\rightarrow 10$ & $8.75 \mathrm{E}+09$ & $\rightarrow 26$ & $6.84 \mathrm{E}+12$ \\
\hline 71 & $2.30 \mathrm{E}+13$ & $\rightarrow 10$ & $7 \mathrm{E}+11$ & $\rightarrow 7$ & +11 & $\rightarrow 6$ & $1 E+10$ & $\rightarrow 21$ & +09 & $\rightarrow 4$ & $2.42 \mathrm{E}+13$ \\
\hline 72 & $1.64 \mathrm{E}+12$ & $\rightarrow 13$ & +11 & $\rightarrow 14$ & +11 & $\rightarrow 12$ & $9 \mathrm{E}+11$ & $\rightarrow$ & -11 & $\rightarrow 1$ & $3.81 \mathrm{E}+12$ \\
\hline 73 & 1.02 & $\rightarrow 8$ & & $\rightarrow 10$ & & $\rightarrow 6$ & -11 & $\rightarrow$ & & $\rightarrow 9$ & -13 \\
\hline 74 & 8 & $\rightarrow 10$ & & $\rightarrow 9$ & & $\rightarrow 8$ & 11 & $\rightarrow$ & & $\rightarrow 7$ & 13 \\
\hline 75 & 2.4 & $\rightarrow 3$ & & $\rightarrow 9$ & & $\rightarrow 10$ & +11 & $\rightarrow 4$ & +10 & $\rightarrow$ & +12 \\
\hline 76 & +12 & $\rightarrow 4$ & 12 & $\rightarrow 10$ & 11 & $\rightarrow 8$ & -11 & $\rightarrow 9$ & 11 & $\rightarrow$ & 4.7 \\
\hline 77 & $1.59 \mathrm{E}+12$ & $\rightarrow 4$ & $E+11$ & $\rightarrow 8$ & +11 & $\rightarrow 9$ & +11 & $\rightarrow 5$ & +11 & $\rightarrow$ & -12 \\
\hline 78 & $3.15 \mathrm{E}+12$ & $\rightarrow 4$ & $2.36 \mathrm{E}+11$ & $\rightarrow 5$ & $3.43 \mathrm{E}+10$ & $\rightarrow 10$ & $4.35 \mathrm{E}+09$ & $\rightarrow 30$ & $3.63 \mathrm{E}+09$ & $\rightarrow 33$ & $3.45 E+12$ \\
\hline 79 & $2.13 E+12$ & $\rightarrow 4$ & $1.11 \mathrm{E}+12$ & $\rightarrow 8$ & $5.37 \mathrm{E}+11$ & $\rightarrow 9$ & $4.87 \mathrm{E}+11$ & $\rightarrow 3$ & $2.99 \mathrm{E}+11$ & $\rightarrow 6$ & $4.81 \mathrm{E}+12$ \\
\hline 80 & $1.92 \mathrm{E}+12$ & $\rightarrow 13$ & $7.00 \mathrm{E}+11$ & $\rightarrow 12$ & $4.97 \mathrm{E}+11$ & $\rightarrow 15$ & $1.31 \mathrm{E}+11$ & $\rightarrow 11$ & $1.24 \mathrm{E}+11$ & $\rightarrow 2$ & $3.38 \mathrm{E}+12$ \\
\hline 81 & $2.30 \mathrm{E}+12$ & $\rightarrow 5$ & $1.13 \mathrm{E}+12$ & $\rightarrow 10$ & $3.82 \mathrm{E}+11$ & $\rightarrow 7$ & $3.67 \mathrm{E}+11$ & $\rightarrow 4$ & $9.47 \mathrm{E}+10$ & $\rightarrow 6$ & $4.29 \mathrm{E}+12$ \\
\hline 82 & $1.32 \mathrm{E}+12$ & $\rightarrow 8$ & $1.01 \mathrm{E}+12$ & $\rightarrow 6$ & +11 & $\rightarrow 4$ & +11 & $\rightarrow 10$ & +11 & $\rightarrow 9$ & $5.00 \mathrm{E}+12$ \\
\hline 83 & $2.19 \mathrm{E}+12$ & $\rightarrow 15$ & +11 & $\rightarrow 12$ & +11 & $\rightarrow 13$ & +11 & $\rightarrow 2$ & & $\rightarrow 14$ & 3.731 \\
\hline 84 & 3.27 & $\rightarrow 5$ & +10 & $\rightarrow 7$ & +09 & $\rightarrow 33$ & +09 & $\rightarrow 30$ & & $\rightarrow 11$ & \\
\hline 85 & $6.02 \mathrm{E}+11$ & $\rightarrow 11$ & $E+11$ & $\rightarrow 12$ & +10 & $\rightarrow 15$ & +10 & $\rightarrow 1$ & & $\rightarrow 14$ & 1.4 \\
\hline 86 & 2.18 & $\rightarrow 5$ & & $\rightarrow 4$ & & $\rightarrow 7$ & & $\rightarrow 10$ & & $\rightarrow 6$ & 5.66 \\
\hline 87 & 2.14 & $\rightarrow 11$ & & $\rightarrow 12$ & & $\rightarrow 13$ & & $\rightarrow$ & & $\rightarrow$ & 12 \\
\hline 88 & 1 & $\rightarrow 10$ & & $\rightarrow 5$ & & $\rightarrow 6$ & & $\rightarrow 7$ & & $\rightarrow$ & 12 \\
\hline 89 & 2 & $\rightarrow 5$ & & $\rightarrow 8$ & 12 & $\rightarrow 10$ & 12 & $\rightarrow 6$ & 11 & $\rightarrow 9$ & 6.3 \\
\hline 90 & 3.97 & $\rightarrow 10$ & 11 & $\rightarrow 8$ & 11 & $\rightarrow 9$ & 10 & $\rightarrow 4$ & 10 & $\rightarrow 3$ & 5.3 \\
\hline 91 & $3.35 \mathrm{E}+11$ & $\rightarrow 13$ & +09 & $\rightarrow 4$ & +09 & $\rightarrow 48$ & +09 & $\rightarrow 40$ & 2.4 & $\rightarrow 38$ & $3.59 \mathrm{E}+11$ \\
\hline 92 & $2.16 \mathrm{E}+12$ & $\rightarrow 11$ & $8.39 \mathrm{E}+11$ & $\rightarrow 13$ & +11 & $\rightarrow 15$ & $5.22 \mathrm{E}+11$ & $\rightarrow 14$ & +11 & $\rightarrow 12$ & $4.83 \mathrm{E}+12$ \\
\hline 93 & $2.63 \mathrm{E}+12$ & $\rightarrow 11$ & $3.58 \mathrm{E}+11$ & $\rightarrow 12$ & $1.93 \mathrm{E}+10$ & $\rightarrow 13$ & $8.96 \mathrm{E}+09$ & $\rightarrow 15$ & $E+09$ & $\rightarrow 4$ & $3.03 \mathrm{E}+12$ \\
\hline 94 & $6.83 \mathrm{E}+11$ & $\rightarrow 11$ & $5.84 \mathrm{E}+11$ & $\rightarrow 14$ & $3.25 \mathrm{E}+11$ & $\rightarrow 12$ & $1.88 \mathrm{E}+11$ & $\rightarrow 15$ & $1.46 \mathrm{E}+10$ & $\rightarrow 35$ & $1.82 \mathrm{E}+12$ \\
\hline 95 & $1.61 \mathrm{E}+12$ & $\rightarrow 6$ & $1.43 \mathrm{E}+12$ & $\rightarrow 10$ & $5.52 \mathrm{E}+11$ & $\rightarrow 7$ & $1.06 \mathrm{E}+11$ & $\rightarrow 5$ & $5.27 \mathrm{E}+10$ & $\rightarrow 4$ & $3.76 \mathrm{E}+12$ \\
\hline 96 & $6.88 \mathrm{E}+09$ & $\rightarrow 5$ & $2.61 E+09$ & $\rightarrow 41$ & +09 & $\rightarrow 48$ & +09 & $\rightarrow 50$ & +09 & $\rightarrow 40$ & $2.14 \mathrm{E}+10$ \\
\hline 97 & $1.03 \mathrm{E}+10$ & $\rightarrow 5$ & +09 & $\rightarrow 48$ & +09 & $\rightarrow 40$ & +08 & $\rightarrow 50$ & +08 & $\rightarrow 84$ & 2.21 \\
\hline 98 & 4.311 & $\rightarrow 12$ & -11 & $\rightarrow 11$ & -11 & $\rightarrow 15$ & -11 & $\rightarrow 14$ & 11 & $\rightarrow 13$ & 6.46 \\
\hline 99 & & $\rightarrow 11$ & & $\rightarrow 12$ & & $\rightarrow 13$ & & $\rightarrow 15$ & & $\rightarrow 40$ & 9.47 \\
\hline 100 & & $\rightarrow 7$ & & $\rightarrow 5$ & 09 & $\rightarrow 60$ & 09 & $\rightarrow 33$ & -09 & $\rightarrow 13$ & 3.3 \\
\hline 101 & & $\rightarrow 7$ & & $\rightarrow 8$ & & $\rightarrow 6$ & & $\rightarrow 10$ & & $\rightarrow$ & 12 \\
\hline 102 & & $\rightarrow 7$ & & $\rightarrow 10$ & & $\rightarrow 6$ & 09 & $\rightarrow 37$ & 09 & $\rightarrow 58$ & 7.0 \\
\hline 103 & 13 & $\rightarrow 11$ & & $\rightarrow 13$ & 11 & $\rightarrow 15$ & 10 & $\rightarrow 12$ & 1. & $\rightarrow$ & 1.9 \\
\hline 104 & 2 & $\rightarrow 14$ & & $\rightarrow 11$ & 11 & $\rightarrow 15$ & 10 & $\rightarrow 12$ & & $\rightarrow 1$ & 8.6 \\
\hline 105 & $4.31 \mathrm{E}+12$ & $\rightarrow 6$ & +11 & $\rightarrow 10$ & +11 & $\rightarrow 8$ & 1.39 & $\rightarrow 9$ & -09 & $\rightarrow 4$ & $4.86 \mathrm{E}+12$ \\
\hline 106 & $2.42 \mathrm{E}+13$ & $\rightarrow 11$ & $9.87 \mathrm{E}+11$ & $\rightarrow 13$ & $4.01 \mathrm{E}+10$ & $\rightarrow 14$ & $3.69 \mathrm{E}+10$ & $\rightarrow 2$ & $E+09$ & $\rightarrow 5$ & $2.53 \mathrm{E}+13$ \\
\hline 107 & $3.81 \mathrm{E}+12$ & $\rightarrow 15$ & $2.27 \mathrm{E}+12$ & $\rightarrow 14$ & $1.64 \mathrm{E}+12$ & $\rightarrow 11$ & $6.20 \mathrm{E}+10$ & $\rightarrow 2$ & $3.76 \mathrm{E}+10$ & $\rightarrow 1$ & $7.85 \mathrm{E}+12$ \\
\hline 108 & $2.32 \mathrm{E}+13$ & $\rightarrow 11$ & $1.79 \mathrm{E}+12$ & $\rightarrow 14$ & $1.35 \mathrm{E}+11$ & $\rightarrow 15$ & $4.52 \mathrm{E}+10$ & $\rightarrow 2$ & $4.22 \mathrm{E}+10$ & $\rightarrow 12$ & $2.53 \mathrm{E}+13$ \\
\hline 109 & $7.20 \mathrm{E}+12$ & $\rightarrow 13$ & $3.15 \mathrm{E}+09$ & $\rightarrow 6$ & $2.48 \mathrm{E}+09$ & $\rightarrow 5$ & $2.47 \mathrm{E}+09$ & $\rightarrow 49$ & $\mathrm{E}+09$ & $\rightarrow 56$ & $7.22 \mathrm{E}+12$ \\
\hline 110 & $2.00 \mathrm{E}+13$ & $\rightarrow 12$ & $5.56 \mathrm{E}+12$ & $\rightarrow 13$ & $9.88 \mathrm{E}+11$ & $\rightarrow 11$ & $5.10 \mathrm{E}+11$ & $\rightarrow 15$ & $E+11$ & $\rightarrow 2$ & $2.73 E+13$ \\
\hline 111 & $1.32 \mathrm{E}+13$ & $\rightarrow 14$ & $1.26 \mathrm{E}+12$ & $\rightarrow 15$ & $1.09 \mathrm{E}+12$ & $\rightarrow 13$ & $3.44 \mathrm{E}+11$ & $\rightarrow 1$ & $E+10$ & $\rightarrow 11$ & $1.60 \mathrm{E}+13$ \\
\hline 112 & $2.99 \mathrm{E}+12$ & $\rightarrow 10$ & $1.25 \mathrm{E}+12$ & $\rightarrow 7$ & $9.25 \mathrm{E}+11$ & $\rightarrow 6$ & $3.01 \mathrm{E}+10$ & $\rightarrow 5$ & $2.34 \mathrm{E}+10$ & $\rightarrow 4$ & $5.24 \mathrm{E}+12$ \\
\hline 113 & $2.18 \mathrm{E}+12$ & $\rightarrow 13$ & $6.25 \mathrm{E}+09$ & $\rightarrow 6$ & $1.76 \mathrm{E}+09$ & $\rightarrow 7$ & $\mathrm{E}+09$ & $\rightarrow 10$ & $\mathrm{E}+09$ & $\rightarrow 48$ & $2.19 \mathrm{E}+12$ \\
\hline 114 & $9.69 \mathrm{E}+09$ & $\rightarrow 7$ & $2.95 \mathrm{E}+09$ & $\rightarrow 48$ & $2.02 \mathrm{E}+09$ & $\rightarrow 66$ & $5.73 \mathrm{E}+08$ & $\rightarrow 100$ & $4.20 \mathrm{E}+08$ & $\rightarrow 5$ & $1.60 \mathrm{E}+10$ \\
\hline 115 & $1.39 \mathrm{E}+13$ & $\rightarrow 12$ & $3.52 \mathrm{E}+12$ & $\rightarrow 13$ & $1.91 \mathrm{E}+12$ & $\rightarrow 14$ & $3.48 \mathrm{E}+11$ & $\rightarrow 11$ & $1.84 \mathrm{E}+11$ & $\rightarrow 15$ & $1.99 \mathrm{E}+13$ \\
\hline 116 & +13 & $\rightarrow 13$ & $E+13$ & $\rightarrow 12$ & +12 & $\rightarrow 15$ & $E+11$ & $\rightarrow 11$ & $E+10$ & $\rightarrow 2$ & $2.53 \mathrm{E}+13$ \\
\hline 117 & $3.29 \mathrm{E}+13$ & $\rightarrow 13$ & $5.22 \mathrm{E}+09$ & $\rightarrow 7$ & $4.33 \mathrm{E}+09$ & $\rightarrow 56$ & $3.62 \mathrm{E}+09$ & $\rightarrow 10$ & $1.63 \mathrm{E}+09$ & $\rightarrow 67$ & $3.29 \mathrm{E}+13$ \\
\hline 118 & $8.35 \mathrm{E}+12$ & $\rightarrow 12$ & $8.03 E+12$ & $\rightarrow 14$ & $1.57 \mathrm{E}+11$ & $\rightarrow 15$ & $1.04 \mathrm{E}+11$ & $\rightarrow 1$ & $7.73 \mathrm{E}+10$ & $\rightarrow 11$ & $1.68 \mathrm{E}+13$ \\
\hline 119 & $4.76 \mathrm{E}+12$ & $\rightarrow 9$ & $3.47 \mathrm{E}+11$ & $\rightarrow 8$ & $2.25 \mathrm{E}+11$ & $\rightarrow 10$ & $2.88 \mathrm{E}+10$ & $\rightarrow 6$ & $2.41 \mathrm{E}+10$ & $\rightarrow 58$ & $5.40 \mathrm{E}+12$ \\
\hline 120 & $1.98 \mathrm{E}+13$ & $\rightarrow 15$ & $5.67 \mathrm{E}+12$ & $\rightarrow 12$ & $7.54 \mathrm{E}+11$ & $\rightarrow 13$ & $2.12 \mathrm{E}+11$ & $\rightarrow 2$ & $1.11 \mathrm{E}+11$ & $\rightarrow 11$ & $2.66 \mathrm{E}+13$ \\
\hline
\end{tabular}


Table 5. continued.

\begin{tabular}{|c|c|c|c|c|c|c|c|c|c|c|c|}
\hline Level & $A^{r}\left(\mathrm{~s}^{-1}\right)$ & $\begin{array}{l}\text { final } \\
\text { level }\end{array}$ & $A^{r}\left(\mathrm{~s}^{-1}\right)$ & $\begin{array}{l}\text { final } \\
\text { level }\end{array}$ & $A^{r}\left(\mathrm{~s}^{-1}\right)$ & $\begin{array}{l}\text { final } \\
\text { level }\end{array}$ & $A^{r}\left(\mathrm{~s}^{-1}\right)$ & $\begin{array}{l}\text { final } \\
\text { level }\end{array}$ & $A^{r}\left(\mathrm{~s}^{-1}\right)$ & $\begin{array}{l}\text { final } \\
\text { level }\end{array}$ & $\sum A^{r}\left(\mathrm{~s}^{-1}\right)$ \\
\hline$\overline{121}$ & $9.83 \mathrm{E}+12$ & $\rightarrow 15$ & $.75 \mathrm{E}+12$ & $\rightarrow 12$ & $1.11 \mathrm{E}+12$ & $\rightarrow 14$ & $71 \mathrm{E}+11$ & $\rightarrow 11$ & $1.35 \mathrm{E}+11$ & $\rightarrow 2$ & $1.43 \mathrm{E}+13$ \\
\hline 122 & $83 \mathrm{E}+13$ & 15 & $89 \mathrm{E}+12$ & $\rightarrow 13$ & $19 \mathrm{E}+12$ & $\rightarrow 14$ & $21 E+11$ & $\rightarrow 11$ & $2.27 \mathrm{E}+11$ & $\rightarrow 2$ & $2.20 \mathrm{E}+13$ \\
\hline 23 & $92 \mathrm{E}+12$ & $\rightarrow 9$ & $.22 \mathrm{E}+12$ & $\rightarrow 8$ & $84 \mathrm{E}+11$ & $\rightarrow 10$ & $39 \mathrm{E}+10$ & $\rightarrow 60$ & $1.02 \mathrm{E}+10$ & $\rightarrow 61$ & $67 \mathrm{E}+12$ \\
\hline 24 & $34 \mathrm{E}+13$ & $\rightarrow 15$ & $4.98 \mathrm{E}+11$ & $\rightarrow 13$ & $44 \mathrm{E}+11$ & $\rightarrow 12$ & $88 \mathrm{E}+10$ & $\rightarrow 2$ & $2.93 \mathrm{E}+10$ & $\rightarrow 11$ & $41 \mathrm{E}+13$ \\
\hline 25 & $55 \mathrm{E}+13$ & $\rightarrow 14$ & $.48 \mathrm{E}+12$ & $\rightarrow 15$ & $1.15 \mathrm{E}+11$ & $\rightarrow 12$ & $6.98 \mathrm{E}+10$ & $\rightarrow 13$ & $1.96 \mathrm{E}+10$ & $\rightarrow 67$ & $.92 \mathrm{E}+13$ \\
\hline 26 & $05 E+11$ & $\rightarrow 2$ & $4.39 \mathrm{E}+11$ & $\rightarrow 18$ & $.39 E+11$ & $\rightarrow 1$ & $2.09 \mathrm{E}+11$ & $\rightarrow 17$ & $1.09 \mathrm{E}+10$ & $\rightarrow 27$ & $1.71 \mathrm{E}+12$ \\
\hline 127 & $15 \mathrm{E}+11$ & $\rightarrow 16$ & $1.32 \mathrm{E}+11$ & $\rightarrow 20$ & $4.43 \mathrm{E}+10$ & $\rightarrow 9$ & $3.89 \mathrm{E}+10$ & $\rightarrow 8$ & $1.25 \mathrm{E}+10$ & $\rightarrow 6$ & $9.58 \mathrm{E}+11$ \\
\hline 128 & $.85 \mathrm{E}+11$ & $\rightarrow 16$ & $1.13 \mathrm{E}+11$ & $\rightarrow 21$ & $4.62 \mathrm{E}+10$ & $\rightarrow 9$ & $3.85 \mathrm{E}+10$ & $\rightarrow 8$ & $1.24 \mathrm{E}+10$ & $\rightarrow 20$ & $9.22 \mathrm{E}+11$ \\
\hline 129 & $32 \mathrm{E}+12$ & $\rightarrow 1$ & $1.36 \mathrm{E}+12$ & $\rightarrow 17$ & $1.04 \mathrm{E}+12$ & $\rightarrow 2$ & $.73 E+11$ & $\rightarrow 18$ & $4.26 \mathrm{E}+10$ & $\rightarrow 23$ & $8.06 \mathrm{E}+12$ \\
\hline 130 & $25 \mathrm{E}+12$ & $\rightarrow 2$ & $63 \mathrm{E}+12$ & $\rightarrow 18$ & $.47 \mathrm{E}+10$ & $\rightarrow 27$ & $48 \mathrm{E}+10$ & $\rightarrow 22$ & $4.59 \mathrm{E}+09$ & $\rightarrow 74$ & $.95 \mathrm{E}+12$ \\
\hline 131 & $17 \mathrm{E}+12$ & $\rightarrow 20$ & $26 \mathrm{E}+11$ & $\rightarrow 21$ & $1.07 \mathrm{E}+10$ & $\rightarrow 58$ & $51 \mathrm{E}+09$ & $\rightarrow 1$ & $3.89 \mathrm{E}+09$ & $\rightarrow 61$ & $3.43 \mathrm{E}+12$ \\
\hline 132 & $3.39 \mathrm{E}+12$ & $\rightarrow 21$ & $1.44 \mathrm{E}+10$ & $\rightarrow 60$ & $3 E+09$ & $\rightarrow 2$ & $70 \mathrm{E}+09$ & $\rightarrow 7$ & $2.26 \mathrm{E}+09$ & $\rightarrow 30$ & $3.42 \mathrm{E}+12$ \\
\hline 133 & $6.96 \mathrm{E}+11$ & $\rightarrow 4$ & $3.23 \mathrm{E}+11$ & $\rightarrow 28$ & 11 & $\rightarrow 25$ & 11 & $\rightarrow 3$ & $8.33 \mathrm{E}+10$ & $\rightarrow 6$ & $1.65 \mathrm{E}+12$ \\
\hline 134 & $E+11$ & $\rightarrow 5$ & $\mathrm{E}+11$ & $\rightarrow 3$ & & 0 & 11 & $\rightarrow 31$ & $1.16 \mathrm{E}+11$ & $\rightarrow 26$ & $1.74 \mathrm{E}+12$ \\
\hline 135 & 1 & $\rightarrow 6$ & $E+11$ & $\rightarrow 8$ & & 2 & & $\rightarrow 29$ & $7.54 \mathrm{E}$ & $\rightarrow 26$ & $1.74 \mathrm{E}+12$ \\
\hline 136 & 1 & $\rightarrow 1$ & $E+11$ & $\rightarrow 19$ & 11 & 35 & $13 \mathrm{E}+10$ & $\rightarrow 23$ & $4.50 \mathrm{E}+10$ & $\rightarrow 2$ & $1.71 \mathrm{E}+12$ \\
\hline 37 & $E+11$ & $\rightarrow 19$ & $\mathrm{E}+11$ & $\rightarrow 1$ & 11 & $\rightarrow 22$ & $46 \mathrm{E}+10$ & $\rightarrow 38$ & $2.90 \mathrm{E}+10$ & $\rightarrow 2$ & $.85 E+11$ \\
\hline 38 & $52 \mathrm{E}+12$ & $\rightarrow 1$ & $4 \mathrm{E}+11$ & $\rightarrow 22$ & $3 E+11$ & $\rightarrow 2$ & $15 \mathrm{E}+11$ & $\rightarrow 23$ & $7.39 \mathrm{E}+10$ & $\rightarrow 41$ & $2.78 \mathrm{E}+12$ \\
\hline 39 & $44 \mathrm{E}+11$ & $\rightarrow 5$ & 11E+11 & $\rightarrow 4$ & $\mathrm{E}+11$ & $\rightarrow 33$ & $06 \mathrm{E}+11$ & $\rightarrow 37$ & $8.14 \mathrm{E}+10$ & $\rightarrow 36$ & $2.13 E+12$ \\
\hline 40 & $06 \mathrm{E}+11$ & $\rightarrow 22$ & $8 \mathrm{E}+11$ & $\rightarrow 1$ & $74 \mathrm{E}+11$ & $\rightarrow 2$ & $66 \mathrm{E}+10$ & $\rightarrow 23$ & $8.25 \mathrm{E}+10$ & $\rightarrow 19$ & $1.54 \mathrm{E}+12$ \\
\hline 41 & $80 \mathrm{E}+11$ & $\rightarrow 22$ & $1.83 \mathrm{E}+11$ & $\rightarrow 2$ & $.84 \mathrm{E}+10$ & $\rightarrow 40$ & $33 \mathrm{E}+10$ & $\rightarrow 41$ & $7.75 \mathrm{E}+09$ & $\rightarrow 38$ & $1.01 \mathrm{E}+12$ \\
\hline 142 & $15 \mathrm{E}+12$ & $\rightarrow 1$ & $4.88 \mathrm{E}+11$ & $\rightarrow 23$ & $1.48 \mathrm{E}+11$ & $\rightarrow 22$ & $43 \mathrm{E}+10$ & $\rightarrow 47$ & $4.66 \mathrm{E}+10$ & $\rightarrow 14$ & $4.05 \mathrm{E}+12$ \\
\hline 143 & $2.88 \mathrm{E}+12$ & $\rightarrow 1$ & $5 \mathrm{E}+11$ & $\rightarrow 23$ & $1.61 \mathrm{E}+11$ & $\rightarrow 22$ & $92 \mathrm{E}+10$ & $\rightarrow 49$ & $4.99 \mathrm{E}+10$ & $\rightarrow 14$ & $3.79 \mathrm{E}+12$ \\
\hline 144 & $30 \mathrm{E}+12$ & $\rightarrow 25$ & $7 E+11$ & $\rightarrow 3$ & $E+11$ & $\rightarrow 4$ & 11 & $\rightarrow 6$ & $1.62 \mathrm{E}+11$ & $\rightarrow 26$ & $3.26 \mathrm{E}+12$ \\
\hline 145 & $70 \mathrm{E}+11$ & $\rightarrow 7$ & $5 \mathrm{E}+11$ & $\rightarrow 39$ & $E+11$ & $\rightarrow 9$ & & $\rightarrow 10$ & $1.10 \mathrm{E}+11$ & $\rightarrow 44$ & $1.93 \mathrm{E}+12$ \\
\hline 146 & $3.35 \mathrm{E}+12$ & $\rightarrow 4$ & $E+11$ & $\rightarrow 28$ & & $\rightarrow 26$ & & $\rightarrow 6$ & $1.05 \mathrm{E}+11$ & $\rightarrow 5$ & $5.66 \mathrm{E}+12$ \\
\hline 147 & & $\rightarrow 4$ & $E+11$ & $\rightarrow 5$ & & 6 & & $\rightarrow 28$ & $3.75 \mathrm{E}+11$ & $\rightarrow 30$ & $4.85 \mathrm{E}+12$ \\
\hline 148 & 3 & $\rightarrow 5$ & & $\rightarrow 30$ & & $\rightarrow 7$ & & $\rightarrow 37$ & 09 & $\rightarrow 33$ & $65 \mathrm{E}+12$ \\
\hline 149 & & $\rightarrow 3$ & & $\rightarrow 4$ & & & & $\rightarrow 26$ & 11 & $\rightarrow 32$ & 12 \\
\hline 50 & 2 & $\rightarrow 3$ & 2 & $\rightarrow 31$ & 1 & $\rightarrow$ & & $\rightarrow 25$ & $1.69 \mathrm{E}-$ & $\rightarrow 26$ & $1.11 \mathrm{E}+13$ \\
\hline 51 & 12 & $\rightarrow 35$ & 1 & $\rightarrow 47$ & 1 & $\rightarrow 42$ & & $\rightarrow 41$ & 9.3 & $\rightarrow 38$ & $3.42 \mathrm{E}+12$ \\
\hline 52 & 12 & $\rightarrow 41$ & 2 & $\rightarrow 38$ & & $\rightarrow 54$ & & $\rightarrow 49$ & 6.5 & $\rightarrow 51$ & $3.37 \mathrm{E}+12$ \\
\hline 153 & $54 \mathrm{E}+12$ & $\rightarrow 8$ & $16 \mathrm{E}+12$ & $\rightarrow 29$ & $1.13 \mathrm{E}+12$ & $\rightarrow 6$ & $1.72 \mathrm{E}$ & $\rightarrow 3$ & $1.10 \mathrm{E}+11$ & $\rightarrow 26$ & $6.52 \mathrm{E}+12$ \\
\hline 54 & $24 \mathrm{E}+12$ & $\rightarrow 2$ & $29 \mathrm{E}+11$ & $\rightarrow 24$ & $2.58 \mathrm{E}+11$ & $\rightarrow 27$ & $3.64 \mathrm{E}$ & $\rightarrow 51$ & $2.82 \mathrm{E}+10$ & $\rightarrow 52$ & $2.14 \mathrm{E}+12$ \\
\hline 55 & $6 \mathrm{E}+11$ & $\rightarrow 24$ & $42 \mathrm{E}+10$ & $\rightarrow 48$ & $2.30 \mathrm{E}+10$ & $\rightarrow 50$ & $93 \mathrm{E}+10$ & $\rightarrow 51$ & $4.38 \mathrm{E}+09$ & $\rightarrow 41$ & $8.38 \mathrm{E}+11$ \\
\hline 156 & $9 \mathrm{E}+12$ & $\rightarrow 6$ & $4 \mathrm{E}+12$ & $\rightarrow 32$ & +12 & $\rightarrow 4$ & 10 & $\rightarrow 5$ & $8.21 \mathrm{E}+10$ & $\rightarrow 10$ & $46 \mathrm{E}+12$ \\
\hline 157 & $7 E+11$ & $\rightarrow 2$ & $9 \mathrm{E}+11$ & $\rightarrow 24$ & $E+11$ & $\rightarrow 27$ & 10 & $\rightarrow 50$ & $2.16 \mathrm{E}+10$ & $\rightarrow 51$ & $.63 E+12$ \\
\hline 58 & $5 \mathrm{E}+12$ & $\rightarrow 2$ & $3 \mathrm{E}+11$ & $\rightarrow 27$ & $2.49 \mathrm{E}+11$ & $\rightarrow 24$ & 10 & $\rightarrow 43$ & $5.81 \mathrm{E}+10$ & $\rightarrow 54$ & $2.87 \mathrm{E}+12$ \\
\hline 59 & 12 & $\rightarrow 43$ & 1 & $\rightarrow 42$ & & $\rightarrow$ & & $\rightarrow 2$ & 6.2 & $\rightarrow 45$ & $3.43 \mathrm{E}+12$ \\
\hline 160 & & $\rightarrow 38$ & 11 & $\rightarrow 41$ & & 9 & & $\rightarrow 40$ & $E+10$ & $\rightarrow 54$ & $3.41 \mathrm{E}+12$ \\
\hline 161 & & $\rightarrow 42$ & +11 & $\rightarrow 41$ & & & & $\rightarrow 47$ & 11 & $\rightarrow 49$ & $3.43 \mathrm{E}+12$ \\
\hline 162 & 2 & $\rightarrow$ & & $\rightarrow$ & & & & $\rightarrow 84$ & 09 & $\rightarrow 5$ & 12 \\
\hline 163 & & $\rightarrow$ & & - & & & & 46 & 11 & $\rightarrow 38$ & 12 \\
\hline 64 & 2 & $\rightarrow 49$ & 1 & $\rightarrow 41$ & & & & $\rightarrow 51$ & 1 & $\rightarrow 40$ & 12 \\
\hline 65 & 2 & $\rightarrow 2$ & 1 & $\rightarrow 27$ & 1 & $\rightarrow$ & & $\rightarrow 56$ & 0 & $\rightarrow 47$ & 3.90 \\
\hline 66 & 2 & $\rightarrow 2$ & 1 & $\rightarrow 27$ & 1 & $\rightarrow$ & & $\rightarrow 15$ & $7.47 \mathrm{E}+10$ & $\rightarrow 59$ & $4.40 \mathrm{E}+12$ \\
\hline 67 & $1.72 \mathrm{E}+12$ & $\rightarrow 33$ & $26 E+09$ & $\rightarrow 96$ & $1.31 \mathrm{E}+09$ & $\rightarrow 109$ & $1.00 \mathrm{E}+09$ & $\rightarrow 91$ & $5.86 \mathrm{E}+08$ & $\rightarrow 24$ & $1.73 \mathrm{E}+12$ \\
\hline 168 & $47 \mathrm{E}+12$ & $\rightarrow 5$ & $41 \mathrm{E}+11$ & $\rightarrow 37$ & $6.01 \mathrm{E}+11$ & $\rightarrow 33$ & $107 \mathrm{E}$ & $\rightarrow 39$ & $5.74 \mathrm{E}+10$ & $\rightarrow 30$ & $8.21 \mathrm{E}+12$ \\
\hline 169 & $97 E+12$ & $\rightarrow 5$ & $12 \mathrm{E}+12$ & $\rightarrow 4$ & $8.18 \mathrm{E}+11$ & $\rightarrow 37$ & $4.71 \mathrm{E}+11$ & $\rightarrow 34$ & $1.28 \mathrm{E}+11$ & $\rightarrow 33$ & $8.97 \mathrm{E}+12$ \\
\hline 170 & $23 \mathrm{E}+12$ & $\rightarrow 4$ & $62 \mathrm{E}+12$ & $\rightarrow 5$ & $5.76 \mathrm{E}+11$ & $\rightarrow 36$ & 11 & $\rightarrow 34$ & $3.77 \mathrm{E}+11$ & $\rightarrow 37$ & $8.68 \mathrm{E}+12$ \\
\hline 171 & $3 \mathrm{E}+12$ & $\rightarrow 4$ & $38 \mathrm{E}+11$ & $\rightarrow 36$ & $6.72 \mathrm{E}+11$ & $\rightarrow 34$ & $5.40 \mathrm{E}+10$ & $\rightarrow 28$ & $1.82 \mathrm{E}+10$ & $\rightarrow 32$ & $7.66 \mathrm{E}+12$ \\
\hline 172 & $3 E+12$ & $\rightarrow 7$ & $0 \mathrm{E}+12$ & $\rightarrow 10$ & 9. & $\rightarrow 6$ & 6.66 & $\rightarrow 36$ & $5.59 \mathrm{E}+11$ & $\rightarrow 5$ & $7.23 \mathrm{E}+12$ \\
\hline 173 & $8 \mathrm{E}+12$ & $\rightarrow 9$ & $\mathrm{E}+12$ & $\rightarrow 10$ & 2 & $\rightarrow 8$ & 11 & $\rightarrow 44$ & $5.45 \mathrm{E}+11$ & $\rightarrow 7$ & $7.36 \mathrm{E}+12$ \\
\hline 174 & $9.12 \mathrm{E}+12$ & $\rightarrow 7$ & $1.38 \mathrm{E}+12$ & $\rightarrow 39$ & $1.69 \mathrm{E}+11$ & $\rightarrow 37$ & $2.65 \mathrm{E}+10$ & $\rightarrow 30$ & $2.58 \mathrm{E}+10$ & $\rightarrow 60$ & $1.08 \mathrm{E}+13$ \\
\hline 175 & $1.66 \mathrm{E}+12$ & $\rightarrow 51$ & $8.69 \mathrm{E}+11$ & $\rightarrow 50$ & $3.85 \mathrm{E}+11$ & $\rightarrow 49$ & $1.75 \mathrm{E}+11$ & $\rightarrow 54$ & $1.07 \mathrm{E}+11$ & $\rightarrow 40$ & $3.42 \mathrm{E}+12$ \\
\hline 176 & +12 & $\rightarrow 50$ & +11 & $\rightarrow 48$ & +11 & $\rightarrow 40$ & 10 & $\rightarrow 56$ & $9.08 \mathrm{E}+09$ & $\rightarrow 84$ & $3.42 \mathrm{E}+12$ \\
\hline 177 & +12 & $\rightarrow 52$ & +12 & $\rightarrow 51$ & $\mathrm{E}+11$ & & & $\rightarrow 49$ & $1.46 \mathrm{E}+11$ & $\rightarrow 50$ & $3.43 \mathrm{E}+12$ \\
\hline 178 & $E+12$ & $\rightarrow 54$ & $3.83 \mathrm{E}+11$ & $\rightarrow 56$ & $3.20 \mathrm{E}+11$ & $\rightarrow 49$ & & $\rightarrow 51$ & $2.33 \mathrm{E}+11$ & $\rightarrow 50$ & $3.38 \mathrm{E}+12$ \\
\hline 179 & $2 \mathrm{E}+12$ & $\rightarrow 48$ & $9.11 \mathrm{E}+08$ & $\rightarrow 33$ & $5.85 \mathrm{E}$ & $\rightarrow 167$ & & $\rightarrow 96$ & $2.79 \mathrm{E}+05$ & $\rightarrow 109$ & $3.42 \mathrm{E}+12$ \\
\hline 180 & 5.39E+12 & $\rightarrow 9$ & $1.10 \mathrm{E}+12$ & $\rightarrow 44$ & $7.28 \mathrm{E}+11$ & $\rightarrow 8$ & $4.82 \mathrm{E}+11$ & $\rightarrow 10$ & $2.75 \mathrm{E}+11$ & $\rightarrow 3$ & $8.71 \mathrm{E}+12$ \\
\hline
\end{tabular}


Table 5. continued.

\begin{tabular}{|c|c|c|c|c|c|c|c|c|c|c|c|}
\hline Level & $A^{r}\left(\mathrm{~s}^{-1}\right)$ & $\begin{array}{l}\text { final } \\
\text { level }\end{array}$ & $A^{r}\left(\mathrm{~s}^{-1}\right)$ & $\begin{array}{l}\text { final } \\
\text { level }\end{array}$ & $A^{r}\left(\mathrm{~s}^{-1}\right)$ & $\begin{array}{l}\text { final } \\
\text { level }\end{array}$ & $A^{r}\left(\mathrm{~s}^{-1}\right)$ & $\begin{array}{l}\text { final } \\
\text { level }\end{array}$ & $A^{r}\left(\mathrm{~s}^{-1}\right)$ & $\begin{array}{l}\text { final } \\
\text { level }\end{array}$ & $\sum A^{r}\left(\mathrm{~s}^{-1}\right)$ \\
\hline$\overline{181}$ & $1.77 \mathrm{E}+12$ & $\rightarrow 52$ & $9.83 \mathrm{E}+11$ & $\rightarrow 53$ & $3.46 \mathrm{E}+11$ & $\rightarrow 51$ & $1.67 \mathrm{E}+11$ & $\rightarrow 59$ & $5.10 \mathrm{E}+10$ & $\rightarrow 47$ & $3.44 \mathrm{E}+12$ \\
\hline 182 & $2.92 \mathrm{E}+12$ & $\rightarrow 56$ & $2.26 \mathrm{E}+11$ & $\rightarrow 40$ & $3.84 \mathrm{E}+10$ & $\rightarrow 66$ & $1.00 \mathrm{E}+10$ & $\rightarrow 7$ & $8.05 E+09$ & $\rightarrow 50$ & $3.21 \mathrm{E}+12$ \\
\hline 183 & $1.66 \mathrm{E}+12$ & $\rightarrow 55$ & $6.17 \mathrm{E}+11$ & $\rightarrow 54$ & $3.15 \mathrm{E}+11$ & $\rightarrow 52$ & $2.84 \mathrm{E}+11$ & $\rightarrow 51$ & $2.48 \mathrm{E}+11$ & $\rightarrow 47$ & $3.39 \mathrm{E}+12$ \\
\hline 184 & $2.64 \mathrm{E}+12$ & $\rightarrow 53$ & $7.46 \mathrm{E}+11$ & $\rightarrow 52$ & $9.98 \mathrm{E}+09$ & $\rightarrow 19$ & $8.79 E+09$ & $\rightarrow 43$ & $7.77 \mathrm{E}+09$ & $\rightarrow 76$ & $3.45 E+12$ \\
\hline 185 & $2.02 \mathrm{E}+12$ & $\rightarrow 59$ & $7.06 \mathrm{E}+11$ & $\rightarrow 55$ & $1.63 \mathrm{E}+11$ & $\rightarrow 47$ & $1.39 \mathrm{E}+11$ & $\rightarrow 52$ & $7.18 \mathrm{E}+10$ & $\rightarrow 53$ & $3.35 \mathrm{E}+12$ \\
\hline 186 & $3.87 \mathrm{E}+11$ & $\rightarrow 6$ & $3.07 \mathrm{E}+11$ & $\rightarrow 61$ & $2.59 \mathrm{E}+11$ & $\rightarrow 10$ & $2.28 \mathrm{E}+11$ & $\rightarrow 9$ & $1.18 \mathrm{E}+11$ & $\rightarrow 58$ & $1.68 \mathrm{E}+12$ \\
\hline 187 & $3.80 \mathrm{E}+11$ & $\rightarrow 10$ & $3.14 \mathrm{E}+11$ & $\rightarrow 60$ & $2.59 \mathrm{E}+11$ & $\rightarrow 7$ & $1.59 \mathrm{E}+11$ & $\rightarrow 58$ & $9.69 \mathrm{E}+10$ & $\rightarrow 6$ & $1.56 \mathrm{E}+12$ \\
\hline 188 & $7.60 \mathrm{E}+11$ & $\rightarrow 2$ & $3.31 \mathrm{E}+11$ & $\rightarrow 45$ & $3.30 \mathrm{E}+11$ & $\rightarrow 46$ & $2.27 \mathrm{E}+11$ & $\rightarrow 1$ & $1.02 \mathrm{E}+11$ & $\rightarrow 67$ & $1.89 \mathrm{E}+12$ \\
\hline 189 & $1.18 \mathrm{E}+12$ & $\rightarrow 2$ & $4.15 E+11$ & $\rightarrow 46$ & $2.87 \mathrm{E}+11$ & $\rightarrow 1$ & $2.23 \mathrm{E}+11$ & $\rightarrow 45$ & $9.18 \mathrm{E}+10$ & $\rightarrow 69$ & $2.37 \mathrm{E}+12$ \\
\hline 190 & $1.20 \mathrm{E}+12$ & $\rightarrow 2$ & $6.04 \mathrm{E}+11$ & $\rightarrow 46$ & $1.03 \mathrm{E}+11$ & $\rightarrow 66$ & $6.68 \mathrm{E}+10$ & $\rightarrow 47$ & $1.94 \mathrm{E}+10$ & $\rightarrow 128$ & $2.09 \mathrm{E}+12$ \\
\hline 191 & $8.99 \mathrm{E}+11$ & $\rightarrow 2$ & $3.48 \mathrm{E}+11$ & $\rightarrow 1$ & $3.40 \mathrm{E}+11$ & $\rightarrow 45$ & $2.91 E+11$ & $\rightarrow 46$ & $9.40 \mathrm{E}+10$ & $\rightarrow 71$ & $2.12 \mathrm{E}+12$ \\
\hline 192 & $5.31 \mathrm{E}+11$ & $\rightarrow 1$ & $4.50 \mathrm{E}+11$ & $\rightarrow 2$ & $4.13 \mathrm{E}+11$ & $\rightarrow 45$ & $1.76 \mathrm{E}+11$ & $\rightarrow 46$ & $1.02 \mathrm{E}+11$ & $\rightarrow 74$ & $1.85 \mathrm{E}+12$ \\
\hline 193 & $2.87 \mathrm{E}+12$ & $\rightarrow 8$ & $2.76 \mathrm{E}+12$ & $\rightarrow 9$ & $1.26 \mathrm{E}+12$ & $\rightarrow 6$ & $9.63 \mathrm{E}+11$ & $\rightarrow 10$ & $8.72 E+11$ & $\rightarrow 57$ & $9.64 \mathrm{E}+12$ \\
\hline 194 & $4.69 \mathrm{E}+12$ & $\rightarrow 6$ & $1.24 \mathrm{E}+12$ & $\rightarrow 58$ & $3.99 \mathrm{E}+11$ & $\rightarrow 7$ & $2.08 \mathrm{E}+11$ & $\rightarrow 60$ & $1.85 \mathrm{E}+11$ & $\rightarrow 61$ & $6.88 \mathrm{E}+12$ \\
\hline 195 & +12 & $\rightarrow 7$ & 1.6 & $\rightarrow 60$ & $2.32 \mathrm{E}+10$ & $\rightarrow 39$ & $2.21 \mathrm{E}+10$ & $\rightarrow 80$ & $2.18 \mathrm{E}+10$ & $\rightarrow 5$ & $4.98 \mathrm{E}+12$ \\
\hline 196 & +12 & $\rightarrow 10$ & & $\rightarrow 61$ & 11 & $\rightarrow 7$ & -11 & $\rightarrow 60$ & -11 & $\rightarrow 6$ & 9.7 \\
\hline 197 & 4.6 & $\rightarrow 8$ & & $\rightarrow 10$ & & $\rightarrow 57$ & & $\rightarrow 62$ & & $\rightarrow 6$ & \\
\hline 198 & 3 & $\rightarrow 10$ & 2. & $\rightarrow 9$ & 11 & $\rightarrow 63$ & 11 & $\rightarrow 61$ & -11 & $\rightarrow 62$ & $7 .{ }^{2}$ \\
\hline 199 & 2 & $\rightarrow 69$ & 4. & $\rightarrow 67$ & $1.22 \mathrm{~F}$ & $\rightarrow 71$ & 10 & $\rightarrow 47$ & -10 & $\rightarrow 55$ & 12 \\
\hline 200 & $2.85 \mathrm{E}+12$ & $\rightarrow 71$ & 3.8 & $\rightarrow 66$ & 4.9 & $\rightarrow 54$ & -10 & $\rightarrow 67$ & -10 & $\rightarrow 49$ & -12 \\
\hline 201 & $2.91 E+12$ & $\rightarrow 67$ & $1.00 \mathrm{E}+11$ & $\rightarrow 71$ & $1.00 \mathrm{E}+11$ & $\rightarrow 66$ & $5.76 \mathrm{E}+10$ & $\rightarrow 54$ & $5.03 E+10$ & $\rightarrow 95$ & $3.35 E+12$ \\
\hline 202 & $3.17 \mathrm{E}+12$ & $\rightarrow 66$ & $7.29 \mathrm{E}+10$ & $\rightarrow 100$ & $5.78 \mathrm{E}+10$ & $\rightarrow 56$ & $4.15 \mathrm{E}+10$ & $\rightarrow 84$ & $1.32 \mathrm{E}+10$ & $\rightarrow 132$ & $3.36 \mathrm{E}+12$ \\
\hline 203 & $2.07 \mathrm{E}+12$ & $\rightarrow 73$ & $4.77 \mathrm{E}+11$ & $\rightarrow 69$ & $4.03 E+11$ & $\rightarrow 74$ & $2.56 \mathrm{E}+11$ & $\rightarrow 70$ & $6.27 E+10$ & $\rightarrow 75$ & $3.46 \mathrm{E}+12$ \\
\hline 204 & $2.29 \mathrm{E}+12$ & $\rightarrow 74$ & $4.88 \mathrm{E}+11$ & $\rightarrow 71$ & $4.65 E+11$ & $\rightarrow 75$ & $4.61 \mathrm{E}+10$ & $\rightarrow 69$ & $2.50 \mathrm{E}+10$ & $\rightarrow 77$ & $3.45 E+12$ \\
\hline 205 & $5.26 \mathrm{E}+11$ & $\rightarrow 11$ & $2.73 E+11$ & $\rightarrow 75$ & $1.81 \mathrm{E}+11$ & $\rightarrow 70$ & $1.60 \mathrm{E}+11$ & $\rightarrow 12$ & +10 & $\rightarrow 77$ & $1.44 \mathrm{E}+12$ \\
\hline 206 & +12 & $\rightarrow 3$ & $3.72 \mathrm{E}+11$ & $\rightarrow 62$ & $3.39 \mathrm{E}+11$ & $\rightarrow 8$ & +11 & $\rightarrow 63$ & 1.50 & $\rightarrow 9$ & $3.19 \mathrm{E}+12$ \\
\hline 207 & +11 & $\rightarrow 11$ & 2.7 & $\rightarrow 78$ & 10 & $\rightarrow 77$ & 7.54 & $\rightarrow 13$ & & $\rightarrow 82$ & 1.37 \\
\hline 208 & +11 & $\rightarrow 12$ & 3.6 & $\rightarrow 14$ & +11 & $\rightarrow 82$ & 8.39 & $\rightarrow 101$ & +10 & $\rightarrow 79$ & +12 \\
\hline 209 & -12 & $\rightarrow 3$ & & $\rightarrow 62$ & 3. & $\rightarrow 8$ & 11 & $\rightarrow 63$ & 11 & $\rightarrow 9$ & 12 \\
\hline 210 & & $\rightarrow 11$ & & $\rightarrow 84$ & & $\rightarrow 13$ & 11 & $\rightarrow 81$ & 11 & $\rightarrow 86$ & \\
\hline 211 & 12 & $\rightarrow 4$ & & $\rightarrow 10$ & 4 & $\rightarrow 64$ & 11 & $\rightarrow 8$ & 11 & $\rightarrow 9$ & \\
\hline 212 & 1 & $\rightarrow 13$ & & $\rightarrow 14$ & & $\rightarrow 12$ & 11 & $\rightarrow 15$ & 11 & $\rightarrow 88$ & -12 \\
\hline 213 & 12 & $\rightarrow 70$ & 4. & $\rightarrow 11$ & 2 & $\rightarrow 12$ & 1.79 & $\rightarrow 75$ & 11 & $\rightarrow 73$ & -12 \\
\hline 214 & $1.59 \mathrm{E}+12$ & $\rightarrow 4$ & 5.0 & $\rightarrow 8$ & 4.8 & $\rightarrow 64$ & +11 & $\rightarrow 9$ & -11 & $\rightarrow 68$ & +12 \\
\hline 215 & $2.05 \mathrm{E}+12$ & $\rightarrow 11$ & $1.14 \mathrm{E}+12$ & $\rightarrow 75$ & $8.97 \mathrm{E}+11$ & $\rightarrow 12$ & $2.75 E+11$ & $\rightarrow 74$ & $1.71 \mathrm{E}+11$ & $\rightarrow 77$ & $4.66 \mathrm{E}+12$ \\
\hline 216 & $2.16 \mathrm{E}+12$ & $\rightarrow 4$ & $6.68 \mathrm{E}+11$ & $\rightarrow 64$ & $1.52 \mathrm{E}+11$ & $\rightarrow 10$ & $9.15 \mathrm{E}+10$ & $\rightarrow 91$ & $1.07 \mathrm{E}+10$ & $\rightarrow 61$ & $3.17 \mathrm{E}+12$ \\
\hline 217 & $8.94 \mathrm{E}+11$ & $\rightarrow 4$ & $8.84 \mathrm{E}+11$ & $\rightarrow 8$ & $3.81 \mathrm{E}+11$ & $\rightarrow 9$ & $3.34 \mathrm{E}+11$ & $\rightarrow 3$ & $3.23 \mathrm{E}+11$ & $\rightarrow 68$ & $3.47 \mathrm{E}+12$ \\
\hline 218 & $1.11 \mathrm{E}+12$ & $\rightarrow 8$ & $4.87 \mathrm{E}+11$ & $\rightarrow 4$ & $4.43 \mathrm{E}+11$ & $\rightarrow 9$ & $3.83 \mathrm{E}+11$ & $\rightarrow 68$ & $3.53 \mathrm{E}+11$ & $\rightarrow 3$ & $3.58 \mathrm{E}+12$ \\
\hline 219 & $2.83 \mathrm{E}+12$ & $\rightarrow 85$ & $1.61 \mathrm{E}+11$ & $\rightarrow 87$ & $1.32 \mathrm{E}+11$ & $\rightarrow 92$ & $8.57 \mathrm{E}+10$ & $\rightarrow 111$ & $3.26 \mathrm{E}+10$ & $\rightarrow 110$ & $3.39 \mathrm{E}+12$ \\
\hline 220 & $2.52 \mathrm{E}+12$ & $\rightarrow 87$ & $3.72 \mathrm{E}+11$ & $\rightarrow 93$ & $2.12 \mathrm{E}+11$ & $\rightarrow 110$ & $1.22 \mathrm{E}+11$ & $\rightarrow 103$ & +10 & $\rightarrow 99$ & $3.32 \mathrm{E}+12$ \\
\hline 221 & +12 & $\rightarrow 5$ & $5.10 \mathrm{E}+11$ & $\rightarrow 65$ & $4.16 \mathrm{E}+11$ & $\rightarrow 10$ & $2.08 \mathrm{E}+11$ & $\rightarrow 6$ & +11 & $\rightarrow 7$ & $3.32 \mathrm{E}+12$ \\
\hline 222 & -12 & $\rightarrow 5$ & & $\rightarrow 65$ & 11 & $\rightarrow 7$ & 10 & $\rightarrow 97$ & 10 & $\rightarrow 96$ & +12 \\
\hline 223 & $2.31 \mathrm{E}+12$ & $\rightarrow 5$ & 11 & $\rightarrow 65$ & $6 \mathrm{E}+11$ & $\rightarrow 7$ & $1.01 \mathrm{E}+11$ & $\rightarrow 4$ & +10 & $\rightarrow 106$ & $3.45 E+12$ \\
\hline 224 & +12 & $\rightarrow 11$ & & $\rightarrow 76$ & & $\rightarrow 77$ & -11 & $\rightarrow 14$ & 11 & $\rightarrow 15$ & +12 \\
\hline 225 & 12 & $\rightarrow 78$ & & $\rightarrow 13$ & & $\rightarrow 81$ & 09 & $\rightarrow 102$ & 09 & $\rightarrow 146$ & +12 \\
\hline 226 & & $\rightarrow 76$ & & $\rightarrow 11$ & & $\rightarrow 79$ & 11 & $\rightarrow 14$ & 11 & $\rightarrow 77$ & 12 \\
\hline 227 & +12 & $\rightarrow 6$ & 12 & $\rightarrow 10$ & 1 & $\rightarrow 72$ & 1.6 & $\rightarrow 8$ & 1. & $\rightarrow 4$ & 3.6 \\
\hline 228 & $2.99 \mathrm{E}+12$ & $\rightarrow 11$ & 9.0 & $\rightarrow 77$ & $E+11$ & $\rightarrow 78$ & +11 & $\rightarrow 75$ & 1.28 & $\rightarrow 13$ & $5.01 \mathrm{E}+12$ \\
\hline 229 & $1.14 \mathrm{E}+12$ & $\rightarrow 10$ & $8.27 \mathrm{E}+11$ & $\rightarrow 6$ & $6.58 \mathrm{E}+11$ & $\rightarrow 5$ & $4.51 \mathrm{E}+11$ & $\rightarrow 72$ & $1.67 \mathrm{E}+11$ & $\rightarrow 65$ & $3.58 \mathrm{E}+12$ \\
\hline 230 & $1.56 \mathrm{E}+12$ & $\rightarrow 10$ & $4.70 \mathrm{E}+11$ & $\rightarrow 6$ & $3.97 \mathrm{E}+11$ & $\rightarrow 72$ & $2.24 \mathrm{E}+11$ & $\rightarrow 8$ & $1.34 \mathrm{E}+11$ & $\rightarrow 4$ & $3.32 \mathrm{E}+12$ \\
\hline 231 & $1.93 \mathrm{E}+12$ & $\rightarrow 12$ & $1.18 \mathrm{E}+12$ & $\rightarrow 14$ & $9.48 \mathrm{E}+11$ & $\rightarrow 79$ & $7.57 \mathrm{E}+11$ & $\rightarrow 11$ & $1.65 \mathrm{E}+11$ & $\rightarrow 82$ & $5.64 \mathrm{E}+12$ \\
\hline 232 & $4.59 \mathrm{E}+12$ & $\rightarrow 12$ & $1.27 \mathrm{E}+12$ & $\rightarrow 11$ & $1.18 \mathrm{E}+12$ & $\rightarrow 82$ & $1.03 \mathrm{E}+11$ & $\rightarrow 86$ & $1.02 \mathrm{E}+11$ & $\rightarrow 77$ & $7.66 \mathrm{E}+12$ \\
\hline 233 & $2.35 \mathrm{E}+12$ & $\rightarrow 92$ & $2.10 \mathrm{E}+11$ & $\rightarrow 93$ & $1.53 \mathrm{E}+11$ & $\rightarrow 85$ & $1.48 \mathrm{E}+11$ & $\rightarrow 106$ & $1.42 \mathrm{E}+11$ & $\rightarrow 87$ & $3.42 \mathrm{E}+12$ \\
\hline 234 & $1.43 \mathrm{E}+12$ & $\rightarrow 99$ & $7.05 \mathrm{E}+11$ & $\rightarrow 93$ & $4.36 \mathrm{E}+11$ & $\rightarrow 91$ & $3.32 \mathrm{E}+11$ & $\rightarrow 87$ & $3.01 \mathrm{E}+11$ & $\rightarrow 103$ & $3.37 \mathrm{E}+12$ \\
\hline 235 & $3.00 \mathrm{E}+12$ & $\rightarrow 91$ & $1.47 \mathrm{E}+11$ & $\rightarrow 96$ & $1.00 \mathrm{E}+11$ & $\rightarrow 117$ & $6.89 \mathrm{E}+10$ & $\rightarrow 109$ & $1.37 \mathrm{E}+10$ & $\rightarrow 113$ & $3.35 \mathrm{E}+12$ \\
\hline 236 & $1.04 \mathrm{E}+12$ & $\rightarrow 99$ & $8.11 \mathrm{E}+11$ & $\rightarrow 93$ & $8.03 E+11$ & $\rightarrow 110$ & $3.94 \mathrm{E}+11$ & $\rightarrow 87$ & $1.41 \mathrm{E}+11$ & $\rightarrow 116$ & $3.27 \mathrm{E}+12$ \\
\hline 237 & $2.23 \mathrm{E}+12$ & $\rightarrow 94$ & $5.70 \mathrm{E}+11$ & $\rightarrow 92$ & $1.46 \mathrm{E}+11$ & $\rightarrow 98$ & $1.38 \mathrm{E}+11$ & $\rightarrow 106$ & $1.20 \mathrm{E}+11$ & $\rightarrow 108$ & $3.42 \mathrm{E}+12$ \\
\hline 238 & $1.03 \mathrm{E}+12$ & $\rightarrow 81$ & $5.08 \mathrm{E}+11$ & $\rightarrow 84$ & $1.30 \mathrm{E}+11$ & $\rightarrow 78$ & $1.12 \mathrm{E}+10$ & $\rightarrow 67$ & $7.85 \mathrm{E}+09$ & $\rightarrow 66$ & $1.72 \mathrm{E}+12$ \\
\hline 239 & $1.68 \mathrm{E}+12$ & $\rightarrow 84$ & $2.08 \mathrm{E}+10$ & $\rightarrow 66$ & $6.64 \mathrm{E}+09$ & $\rightarrow 167$ & $5.29 \mathrm{E}+09$ & $\rightarrow 50$ & $4.17 \mathrm{E}+09$ & $\rightarrow 148$ & $1.72 \mathrm{E}+12$ \\
\hline 240 & $2.03 E+12$ & $\rightarrow 98$ & $5.88 \mathrm{E}+11$ & $\rightarrow 111$ & $2.91 \mathrm{E}+11$ & $\rightarrow 99$ & $1.46 \mathrm{E}+11$ & $\rightarrow 106$ & $6.08 \mathrm{E}+10$ & $\rightarrow 103$ & $3.35 E+12$ \\
\hline
\end{tabular}


Table 5. continued.

\begin{tabular}{|c|c|c|c|c|c|c|c|c|c|c|c|}
\hline Level & $A^{r}\left(\mathrm{~s}^{-1}\right)$ & $\begin{array}{l}\text { final } \\
\text { level }\end{array}$ & $A^{r}\left(\mathrm{~s}^{-1}\right)$ & $\begin{array}{l}\text { final } \\
\text { level }\end{array}$ & $A^{r}\left(\mathrm{~s}^{-1}\right)$ & $\begin{array}{l}\text { final } \\
\text { level }\end{array}$ & $A^{r}\left(\mathrm{~s}^{-1}\right)$ & $\begin{array}{l}\text { final } \\
\text { level }\end{array}$ & $A^{r}\left(\mathrm{~s}^{-1}\right)$ & $\begin{array}{l}\text { final } \\
\text { level }\end{array}$ & $\sum A^{r}$ \\
\hline 241 & $1.95 \mathrm{E}+12$ & $\rightarrow 13$ & $57 \mathrm{E}+11$ & $\rightarrow 11$ & $3.00 \mathrm{E}+11$ & $\rightarrow 15$ & $.55 \mathrm{E}+11$ & $\rightarrow 102$ & $2.12 \mathrm{E}+11$ & $\rightarrow 100$ & $3.95 \mathrm{E}+12$ \\
\hline 42 & $1 \mathrm{E}+12$ & 15 & $40 \mathrm{E}+11$ & $\rightarrow 95$ & $38 \mathrm{E}+11$ & $\rightarrow 112$ & $50 \mathrm{E}+11$ & $\rightarrow 11$ & $.47 \mathrm{E}+11$ & $\rightarrow 12$ & $15 E+12$ \\
\hline 43 & $E+12$ & 11 & $23 \mathrm{E}+11$ & $\rightarrow 12$ & $45 \mathrm{E}+11$ & $\rightarrow 81$ & $3.36 \mathrm{E}+11$ & $\rightarrow 15$ & $17 \mathrm{E}+11$ & $\rightarrow 89$ & $18 \mathrm{E}+12$ \\
\hline 44 & $13 \mathrm{E}+12$ & $\rightarrow 11$ & $.68 \mathrm{E}+11$ & $\rightarrow 86$ & $60 \mathrm{E}+11$ & $\rightarrow 13$ & $.71 \mathrm{E}+11$ & $\rightarrow 89$ & $40 \mathrm{E}+11$ & $\rightarrow 81$ & $41 \mathrm{E}+12$ \\
\hline 45 & $40 \mathrm{E}+12$ & $\rightarrow 11$ & $8.92 \mathrm{E}+11$ & $\rightarrow 86$ & $3.29 \mathrm{E}+11$ & $\rightarrow 14$ & $3.17 \mathrm{E}+11$ & $\rightarrow 89$ & $.08 \mathrm{E}+11$ & $\rightarrow 77$ & $.04 \mathrm{E}+13$ \\
\hline 46 & $31 E+12$ & $\rightarrow 14$ & $.14 E+11$ & $\rightarrow 11$ & $6.35 \mathrm{E}+11$ & $\rightarrow 90$ & $5.52 \mathrm{E}+11$ & $\rightarrow 15$ & $.16 \mathrm{E}+11$ & $\rightarrow 79$ & $7.48 \mathrm{E}+12$ \\
\hline 47 & $90 \mathrm{E}+12$ & $\rightarrow 13$ & $1.26 \mathrm{E}+12$ & $\rightarrow 88$ & $1.91 \mathrm{E}+11$ & $\rightarrow 102$ & $8.65 \mathrm{E}+10$ & $\rightarrow 84$ & $.87 \mathrm{E}+10$ & $\rightarrow 81$ & $9.56 \mathrm{E}+12$ \\
\hline 48 & $.79 \mathrm{E}+12$ & $\rightarrow 14$ & $.59 \mathrm{E}+11$ & $\rightarrow 15$ & $4.97 \mathrm{E}+11$ & $\rightarrow 90$ & $4.83 \mathrm{E}+11$ & $\rightarrow 13$ & $.75 E+11$ & $\rightarrow 82$ & $8.05 \mathrm{E}+12$ \\
\hline 49 & $70 \mathrm{E}+12$ & $\rightarrow 12$ & $.82 \mathrm{E}+12$ & $\rightarrow 13$ & $88 \mathrm{E}+11$ & $\rightarrow 15$ & $1.88 \mathrm{E}+11$ & $\rightarrow 89$ & $.54 \mathrm{E}+11$ & $\rightarrow 86$ & $7.90 \mathrm{E}+12$ \\
\hline 50 & $30 \mathrm{E}+12$ & $\rightarrow 96$ & $38 \mathrm{E}+11$ & $\rightarrow 97$ & $59 \mathrm{E}+11$ & $\rightarrow 109$ & $2.45 \mathrm{E}+11$ & $\rightarrow 91$ & $.24 \mathrm{E}+11$ & $\rightarrow 117$ & $3.34 \mathrm{E}+12$ \\
\hline 51 & $34 \mathrm{E}+12$ & $\rightarrow 97$ & $24 \mathrm{E}+10$ & $\rightarrow 114$ & $.59 \mathrm{E}+09$ & $\rightarrow 179$ & $3.76 \mathrm{E}+09$ & $\rightarrow 162$ & $.49 \mathrm{E}+08$ & $\rightarrow 84$ & $3.36 \mathrm{E}+12$ \\
\hline 252 & 12 & $\rightarrow 108$ & $6.74 \mathrm{E}+11$ & $\rightarrow 106$ & $2.33 \mathrm{E}+11$ & $\rightarrow 104$ & $1.27 \mathrm{E}+11$ & $\rightarrow 107$ & $01 \mathrm{E}+10$ & $\rightarrow 94$ & $3.41 \mathrm{E}+12$ \\
\hline 53 & 2 & 103 & $61 \mathrm{E}-$ & $\rightarrow 96$ & $7.24 \mathrm{E}+11$ & $\rightarrow 93$ & $3.10 \mathrm{E}-$ & $\rightarrow 110$ & $40 \mathrm{E}+10$ & $\rightarrow 91$ & +12 \\
\hline 54 & & 6 & 1 & $\rightarrow 108$ & $28 \mathrm{E}+11$ & $\rightarrow 103$ & $1.91 \mathrm{E}$ & 4 & 11 & $\rightarrow 92$ & $2 \mathrm{E}+12$ \\
\hline 55 & & & & $\rightarrow 103$ & 1 & $\rightarrow 93$ & 1 & 98 & 11 & 96 & \\
\hline 56 & +12 & 109 & $E+11$ & $\rightarrow 117$ & $68 \mathrm{E}+11$ & $\rightarrow 96$ & $96 \mathrm{E}+11$ & $\rightarrow 97$ & +10 & 91 & $.25 \mathrm{E}+12$ \\
\hline 57 & $E+12$ & 7 & $23 \mathrm{E}+11$ & $\rightarrow 111$ & 04E+11 & $\rightarrow 104$ & $3.88 \mathrm{E}+11$ & $\rightarrow 108$ & +11 & $\rightarrow 98$ & +12 \\
\hline 58 & $\mathrm{E}+12$ & 7110 & $31 \mathrm{E}+11$ & $\rightarrow 103$ & $29 \mathrm{E}+11$ & $\rightarrow 109$ & $2.64 \mathrm{E}+11$ & $\rightarrow 99$ & $\mathrm{OE}+11$ & $\rightarrow 93$ & 12 \\
\hline 59 & $59 \mathrm{E}+12$ & $\rightarrow 111$ & $24 \mathrm{E}+11$ & $\rightarrow 98$ & $37 \mathrm{E}+11$ & $\rightarrow 110$ & $1.85 \mathrm{E}+11$ & $\rightarrow 103$ & $8 \mathrm{E}+11$ & $\rightarrow 93$ & $32 \mathrm{E}+12$ \\
\hline 50 & $\mathrm{E}+11$ & $\rightarrow 2$ & $07 \mathrm{E}+11$ & $\rightarrow 18$ & $50 \mathrm{E}+11$ & $\rightarrow 1$ & $1.44 \mathrm{E}+11$ & $\rightarrow 128$ & $96 \mathrm{E}+10$ & $\rightarrow 17$ & +11 \\
\hline 51 & $16 \mathrm{E}+12$ & $\rightarrow 6$ & $9.44 \mathrm{E}+11$ & $\rightarrow 10$ & $04 \mathrm{E}+11$ & $\rightarrow 83$ & $1.96 \mathrm{E}+11$ & $\rightarrow 7$ & $.00 \mathrm{E}+10$ & $\rightarrow 113$ & $3.25 \mathrm{E}+12$ \\
\hline 52 & $78 \mathrm{E}+12$ & $\rightarrow 7$ & $66 \mathrm{E}+11$ & $\rightarrow 80$ & $31 \mathrm{E}+11$ & $\rightarrow 6$ & $3.63 \mathrm{E}+11$ & $\rightarrow 10$ & $.93 \mathrm{E}+11$ & $\rightarrow 83$ & $3.61 \mathrm{E}+12$ \\
\hline 63 & $10 \mathrm{E}+12$ & $\rightarrow 7$ & $.90 \mathrm{E}+11$ & $\rightarrow 80$ & $38 \mathrm{E}+11$ & $\rightarrow 5$ & $8.80 \mathrm{E}+10$ & $\rightarrow 114$ & $1.54 \mathrm{E}+10$ & $\rightarrow 117$ & $3.09 \mathrm{E}+12$ \\
\hline 64 & 12 & $\rightarrow 7$ & $8 \mathrm{E}+11$ & $\rightarrow 80$ & $37 \mathrm{E}+11$ & $\rightarrow 10$ & $1.06 \mathrm{E}+11$ & $\rightarrow 5$ & +10 & $\rightarrow 83$ & $3.53 \mathrm{E}+12$ \\
\hline 65 & & $\rightarrow 6$ & $97 \mathrm{E}+11$ & $\rightarrow 10$ & $48 \mathrm{E}+11$ & $\rightarrow 83$ & $6.46 \mathrm{E}+10$ & $\rightarrow 72$ & +10 & $\rightarrow 122$ & $3.47 \mathrm{E}+12$ \\
\hline 66 & & $\rightarrow 16$ & $56 \mathrm{E}+11$ & $\rightarrow 126$ & $32 \mathrm{E}+10$ & $\rightarrow 129$ & $E+10$ & $\rightarrow 20$ & +10 & $\rightarrow 8$ & \\
\hline 67 & & 6 & & $\rightarrow 126$ & 10 & $\rightarrow 130$ & & 21 & 10 & $\rightarrow 9$ & \\
\hline 68 & & & & $\rightarrow 6$ & 1 & $\rightarrow 7$ & & & & $\rightarrow 80$ & \\
\hline 69 & & $\rightarrow$ & & $\rightarrow 13$ & & $\rightarrow 10$ & & & & 67 & \\
\hline 70 & & $\rightarrow 1$ & & $\rightarrow 17$ & 1 & $\rightarrow 2$ & 11 & $\rightarrow$ & 11 & $\rightarrow 18$ & 12 \\
\hline 71 & & $\rightarrow 100$ & 0 & $\rightarrow 66$ & 9 & $\rightarrow 84$ & 9 & $\rightarrow 167$ & 09 & $\rightarrow 195$ & 12 \\
\hline 72 & & $\rightarrow 2$ & 1 & $\rightarrow 18$ & 1 & $\rightarrow 128$ & 0 & $\rightarrow 12$ & 10 & $\rightarrow 27$ & 12 \\
\hline 73 & -12 & $\rightarrow 13$ & $39 \mathrm{E}+12$ & $\rightarrow 12$ & $21 \mathrm{E}+11$ & $\rightarrow 102$ & $4.65 E+11$ & $\rightarrow 101$ & +11 & $\rightarrow 88$ & +12 \\
\hline 74 & $E+12$ & $\rightarrow 12$ & $41 \mathrm{E}+12$ & $\rightarrow 13$ & $77 \mathrm{E}+11$ & $\rightarrow 101$ & $3.06 \mathrm{E}+11$ & $\rightarrow 14$ & $5 E+11$ & $\rightarrow 102$ & $8.20 \mathrm{E}+12$ \\
\hline 75 & $58 \mathrm{E}+12$ & $\rightarrow 13$ & $55 \mathrm{E}+11$ & $\rightarrow 102$ & $3.43 \mathrm{E}+11$ & $\rightarrow 100$ & $2.07 \mathrm{E}+11$ & $\rightarrow 95$ & $19 \mathrm{E}+11$ & $\rightarrow 88$ & $8.25 \mathrm{E}+12$ \\
\hline 76 & +12 & $\rightarrow 12$ & +12 & $\rightarrow 14$ & $74 \mathrm{E}+11$ & $\rightarrow 105$ & $4.67 \mathrm{E}+11$ & $\rightarrow 101$ & +11 & $\rightarrow 90$ & $19 E+12$ \\
\hline 77 & +12 & $\rightarrow 20$ & $72 \mathrm{E}+11$ & $\rightarrow 129$ & $71 \mathrm{E}+10$ & $\rightarrow 21$ & $4.09 \mathrm{E}+10$ & $\rightarrow 130$ & +09 & $\rightarrow 6$ & $.80 \mathrm{E}+12$ \\
\hline 78 & -12 & $\rightarrow 21$ & 1 & $\rightarrow 130$ & $46 \mathrm{E}+09$ & $\rightarrow 7$ & $4.88 \mathrm{E}+09$ & $\rightarrow 2$ & +09 & $\rightarrow 116$ & $1.79 \mathrm{E}+12$ \\
\hline 79 & & $\rightarrow 131$ & & $\rightarrow 132$ & & $\rightarrow 67$ & & $\rightarrow 20$ & & $\rightarrow$ & $E+12$ \\
\hline 30 & & $\rightarrow 132$ & & $\rightarrow 66$ & $E+09$ & $\rightarrow 21$ & $E+08$ & & -08 & $\rightarrow 100$ & 12 \\
\hline 81 & & - & & $\rightarrow 12$ & 1 & 12 & 11 & & & $\rightarrow$ & -12 \\
\hline 82 & & & & $\rightarrow 12$ & & 2 & & & & 01 & 13 \\
\hline 83 & & & & $\rightarrow 117$ & & & & & & 96 & 12 \\
\hline 34 & & & & $\rightarrow 1$ & & $\rightarrow 1$ & 1 & & & $\rightarrow$ & \\
\hline 35 & 2 & 5 & 1 & $\rightarrow 1$ & 1 & $\rightarrow 120$ & 1 & $\rightarrow 99$ & 10 & $\rightarrow$ & 12 \\
\hline 86 & & $\rightarrow$ & 1 & $\rightarrow 112$ & 1 & $\rightarrow 13$ & 1 & $\rightarrow 14$ & 11 & $\rightarrow 105$ & 9.7 \\
\hline 37 & $9 \mathrm{E}+12$ & $\rightarrow 120$ & $21 \mathrm{E}+11$ & $\rightarrow 113$ & $18 E+11$ & $\rightarrow 116$ & $1.14 \mathrm{E}+11$ & $\rightarrow 110$ & $7.37 \mathrm{E}+10$ & $\rightarrow 109$ & $3.34 \mathrm{E}+12$ \\
\hline 38 & $0 \mathrm{E}+12$ & $\rightarrow 117$ & $41 \mathrm{E}+12$ & $\rightarrow 113$ & $2.52 \mathrm{E}+11$ & $\rightarrow 109$ & $8.75 \mathrm{E}+10$ & $\rightarrow 114$ & $9.43 \mathrm{E}+09$ & $\rightarrow 91$ & $3.27 \mathrm{E}+12$ \\
\hline 89 & $3.27 \mathrm{E}+12$ & $\rightarrow 114$ & $.82 \mathrm{E}+10$ & $\rightarrow 97$ & $3.43 \mathrm{E}+09$ & $\rightarrow 202$ & $3.00 \mathrm{E}+09$ & $\rightarrow 179$ & $8.35 \mathrm{E}+08$ & $\rightarrow 100$ & $3.29 \mathrm{E}+12$ \\
\hline 90 & $45 \mathrm{E}+12$ & $\rightarrow 115$ & $.29 \mathrm{E}+12$ & $\rightarrow 118$ & $53 \mathrm{E}+11$ & $\rightarrow 116$ & $1.39 \mathrm{E}+11$ & $\rightarrow 107$ & $7.92 \mathrm{~F}$ & $\rightarrow 122$ & $3.44 \mathrm{E}+12$ \\
\hline 91 & $3 \mathrm{E}+12$ & $\rightarrow 122$ & $5.78 \mathrm{E}+11$ & $\rightarrow 120$ & $22 \mathrm{E}+11$ & $\rightarrow 116$ & $6.78 \mathrm{E}+10$ & $\rightarrow 113$ & 10 & $\rightarrow 110$ & $3.37 \mathrm{E}+12$ \\
\hline 292 & $6 \mathrm{E}+12$ & $\rightarrow 121$ & $7.04 \mathrm{E}+11$ & $\rightarrow 118$ & $4.08 \mathrm{E}+11$ & $\rightarrow 115$ & $3.33 \mathrm{E}+11$ & $\rightarrow 122$ & $47 \mathrm{E}+10$ & $\rightarrow 104$ & $3.41 \mathrm{E}+12$ \\
\hline 293 & $9 \mathrm{E}+12$ & $\rightarrow 121$ & $24 \mathrm{E}+12$ & $\rightarrow 122$ & $75 \mathrm{E}+11$ & $\rightarrow 118$ & $1.63 \mathrm{E}+11$ & $\rightarrow 107$ & 11 & $\rightarrow 116$ & $3.40 \mathrm{E}+12$ \\
\hline 294 & -11 & $\rightarrow 123$ & $2.16 \mathrm{E}+11$ & $\rightarrow 119$ & $1.20 \mathrm{E}+11$ & $\rightarrow 14$ & $7.60 \mathrm{E}+10$ & $\rightarrow 15$ & $3.51 \mathrm{E}+10$ & $\rightarrow 112$ & $9.63 \mathrm{E}+11$ \\
\hline 295 & $2.04 \mathrm{E}+12$ & $\rightarrow 9$ & $4.61 \mathrm{E}+11$ & $\rightarrow 8$ & $4.58 \mathrm{E}+11$ & $\rightarrow 104$ & $2.23 \mathrm{E}+11$ & $\rightarrow 107$ & $1.36 \mathrm{E}+11$ & $\rightarrow 125$ & $3.41 \mathrm{E}+12$ \\
\hline 296 & +12 & $\rightarrow 9$ & +11 & $\rightarrow 8$ & $E+11$ & $\rightarrow 104$ & $2.27 \mathrm{E}+11$ & $\rightarrow 107$ & $1.15 \mathrm{E}+11$ & $\rightarrow 124$ & $3.20 \mathrm{E}+12$ \\
\hline 297 & +12 & $\rightarrow 15$ & $E+12$ & $\rightarrow 123$ & $9.80 \mathrm{E}+10$ & $\rightarrow 12$ & $3.35 \mathrm{E}+10$ & $\rightarrow 2$ & $1.76 \mathrm{E}+10$ & $\rightarrow 13$ & $5.60 \mathrm{E}+12$ \\
\hline 98 & +12 & $\rightarrow 1$ & +12 & $\rightarrow 119$ & +11 & $\rightarrow 1$ & $2.76 \mathrm{E}+11$ & $\rightarrow 123$ & $2.02 \mathrm{E}+10$ & $\rightarrow 90$ & $7.39 \mathrm{E}+12$ \\
\hline 299 & $E+12$ & $\rightarrow 125$ & $2.15 \mathrm{E}+11$ & $\rightarrow 124$ & $8.06 \mathrm{E}+10$ & $\rightarrow 111$ & $3.87 \mathrm{E}+10$ & $\rightarrow 115$ & $2.54 \mathrm{E}+10$ & $\rightarrow 122$ & $3.35 \mathrm{E}+12$ \\
\hline 300 & $3.18 \mathrm{E}+12$ & $\rightarrow 124$ & $7.59 \mathrm{E}+10$ & $\rightarrow 120$ & $3.01 \mathrm{E}+10$ & $\rightarrow 110$ & $2.08 \mathrm{E}+10$ & $\rightarrow 116$ & $1.38 \mathrm{E}+10$ & $\rightarrow 202$ & $3.36 \mathrm{E}+12$ \\
\hline
\end{tabular}


Table 5. continued.

\begin{tabular}{|c|c|c|c|c|c|c|c|c|c|c|c|}
\hline Level & $A^{r}\left(\mathrm{~s}^{-1}\right)$ & $\begin{array}{l}\text { final } \\
\text { level }\end{array}$ & $A^{r}\left(\mathrm{~s}^{-1}\right)$ & $\begin{array}{l}\text { final } \\
\text { level }\end{array}$ & $A^{r}\left(\mathrm{~s}^{-1}\right)$ & $\begin{array}{l}\text { final } \\
\text { level }\end{array}$ & $A^{r}\left(\mathrm{~s}^{-1}\right)$ & $\begin{array}{l}\text { final } \\
\text { level }\end{array}$ & $A^{r}\left(\mathrm{~s}^{-1}\right)$ & $\begin{array}{l}\text { final } \\
\text { level }\end{array}$ & $\sum A^{r}\left(\mathrm{~s}^{-1}\right)$ \\
\hline$\overline{301}$ & $2.49 \mathrm{E}+11$ & $\rightarrow 4$ & $1.70 \mathrm{E}+11$ & $\rightarrow 28$ & $1.22 \mathrm{E}+11$ & $\rightarrow 137$ & $74 \mathrm{E}+10$ & $\rightarrow 6$ & $8.72 \mathrm{E}+10$ & $\rightarrow 25$ & $9.85 \mathrm{E}+11$ \\
\hline 302 & $33 \mathrm{E}+11$ & $\rightarrow 5$ & $71 E+11$ & $\rightarrow 3$ & $1.41 \mathrm{E}+11$ & $\rightarrow 30$ & $12 \mathrm{E}+11$ & $\rightarrow 141$ & $11 \mathrm{E}+10$ & $\rightarrow 31$ & $.02 \mathrm{E}+12$ \\
\hline 03 & $E+11$ & $\rightarrow 1$ & $36 \mathrm{E}+11$ & $\rightarrow 19$ & $2 \mathrm{E}+11$ & $\rightarrow 133$ & $03 \mathrm{E}+10$ & $\rightarrow 144$ & $01 \mathrm{E}+10$ & $\rightarrow 23$ & $.21 \mathrm{E}+12$ \\
\hline 04 & $3 E+11$ & $\rightarrow 6$ & $61 \mathrm{E}+11$ & $\rightarrow 8$ & $1.38 \mathrm{E}+11$ & $\rightarrow 32$ & $10 \mathrm{E}+11$ & $\rightarrow 143$ & $9.62 \mathrm{E}+10$ & $\rightarrow 29$ & $.14 \mathrm{E}+12$ \\
\hline 05 & $04 E+11$ & $\rightarrow 1$ & $3.40 \mathrm{E}+11$ & $\rightarrow 19$ & $1.47 \mathrm{E}+11$ & $\rightarrow 133$ & $5.05 \mathrm{E}+10$ & $\rightarrow 146$ & $3.97 \mathrm{E}+10$ & $\rightarrow 23$ & $1.09 \mathrm{E}+12$ \\
\hline 06 & $33 E+11$ & $\rightarrow 1$ & $2.75 E+11$ & $\rightarrow 22$ & $1.78 \mathrm{E}+11$ & $\rightarrow 2$ & $1.16 \mathrm{E}+11$ & $\rightarrow 134$ & $9.53 \mathrm{E}+10$ & $\rightarrow 23$ & $1.44 \mathrm{E}+12$ \\
\hline 07 & $52 \mathrm{E}+11$ & $\rightarrow 25$ & $5.23 \mathrm{E}+11$ & $\rightarrow 3$ & $2.68 \mathrm{E}+11$ & $\rightarrow 136$ & $2.63 \mathrm{E}+11$ & $\rightarrow 4$ & $1.77 \mathrm{E}+11$ & $\rightarrow 8$ & $2.30 \mathrm{E}+12$ \\
\hline 08 & $70 \mathrm{E}+11$ & $\rightarrow 22$ & $1.50 \mathrm{E}+11$ & $\rightarrow 134$ & $1.44 \mathrm{E}+11$ & $\rightarrow 2$ & $3.09 \mathrm{E}+10$ & $\rightarrow 150$ & $2.85 \mathrm{E}+10$ & $\rightarrow 43$ & $8.29 \mathrm{E}+11$ \\
\hline 309 & $76 \mathrm{E}+11$ & $\rightarrow 22$ & $.52 \mathrm{E}+11$ & $\rightarrow 134$ & $1.46 \mathrm{E}+11$ & $\rightarrow 2$ & $4.81 \mathrm{E}+10$ & $\rightarrow 148$ & $3.83 \mathrm{E}+10$ & $\rightarrow 40$ & $8.18 \mathrm{E}+11$ \\
\hline 310 & $00 \mathrm{E}+12$ & $\rightarrow 4$ & $6.21 \mathrm{E}+11$ & $\rightarrow 28$ & $5.85 \mathrm{E}+11$ & $\rightarrow 6$ & $.84 \mathrm{E}+11$ & $\rightarrow 137$ & $1.52 \mathrm{E}+11$ & $\rightarrow 26$ & $3.79 \mathrm{E}+12$ \\
\hline 311 & $30 \mathrm{E}+12$ & $\rightarrow 1$ & $3.16 \mathrm{E}+11$ & $\rightarrow 23$ & $1.43 \mathrm{E}+11$ & $\rightarrow 135$ & $6.20 \mathrm{E}+10$ & $\rightarrow 153$ & $5.02 \mathrm{E}+10$ & $\rightarrow 19$ & $2.54 \mathrm{E}+12$ \\
\hline 312 & $35 E+12$ & $\rightarrow 1$ & $2.45 \mathrm{E}-$ & $\rightarrow 23$ & $1.11 \mathrm{E}+11$ & $\rightarrow 135$ & $8.98 \mathrm{E}+10$ & $\rightarrow 22$ & $5.39 \mathrm{E}+10$ & $\rightarrow 14$ & $2.12 \mathrm{E}+12$ \\
\hline 13 & $E+11$ & $\rightarrow 35$ & 5 & $\rightarrow 144$ & 1. & $\rightarrow 47$ & & $\rightarrow 42$ & +10 & $\rightarrow 153$ & $1.79 \mathrm{E}+12$ \\
\hline 14 & & $\rightarrow 41$ & 1 & $\rightarrow 146$ & & $\rightarrow 38$ & & & -10 & $\rightarrow 54$ & $1.75 \mathrm{E}+12$ \\
\hline 15 & & $\rightarrow 151$ & & $\rightarrow 152$ & & $\rightarrow 163$ & & $\rightarrow$ & 08 & $\rightarrow 35$ & 12 \\
\hline 16 & $\mathrm{E}+12$ & $\rightarrow 152$ & $68 \mathrm{E}-$ & $\rightarrow 160$ & $1.66 \mathrm{E}+09$ & $\rightarrow 164$ & 09 & $\rightarrow 91$ & 7.801 & $\rightarrow 155$ & $.01 \mathrm{E}+12$ \\
\hline 17 & $E+11$ & $\rightarrow 5$ & 4.96 & $\rightarrow 26$ & +11 & $\rightarrow 4$ & $2.06 \mathrm{E}+11$ & $\rightarrow 138$ & 1.78 & $\rightarrow 30$ & $2.28 \mathrm{E}+12$ \\
\hline 18 & $2 E+12$ & $\rightarrow 3$ & $4.68 \mathrm{E}+11$ & $\rightarrow 4$ & $3.26 \mathrm{E}+11$ & $\rightarrow 31$ & $2.34 \mathrm{E}+11$ & $\rightarrow 26$ & $1.61 \mathrm{E}+11$ & $\rightarrow 32$ & $4.22 \mathrm{E}+12$ \\
\hline 19 & $31 \mathrm{E}+12$ & $\rightarrow 5$ & $7.44 \mathrm{E}+11$ & $\rightarrow 30$ & $3.47 \mathrm{E}+11$ & $\rightarrow 7$ & $3.33 \mathrm{E}+11$ & $\rightarrow 141$ & $9.66 \mathrm{E}+10$ & $\rightarrow 37$ & $3.36 \mathrm{E}+12$ \\
\hline 20 & $38 \mathrm{E}+12$ & $\rightarrow 3$ & $6.09 \mathrm{E}+11$ & $\rightarrow 31$ & $2.08 \mathrm{E}+11$ & $\rightarrow 140$ & $1.34 \mathrm{E}+11$ & $\rightarrow 9$ & $1.10 \mathrm{E}+11$ & $\rightarrow 4$ & $5.37 \mathrm{E}+12$ \\
\hline 21 & $85 \mathrm{E}+12$ & $\rightarrow 8$ & $6.08 \mathrm{E}+11$ & $\rightarrow 29$ & $4.33 \mathrm{E}+11$ & $\rightarrow 6$ & $2.29 \mathrm{E}+11$ & $\rightarrow 142$ & $6.20 \mathrm{E}+10$ & $\rightarrow 26$ & $3.51 \mathrm{E}+12$ \\
\hline 322 & $60 \mathrm{E}+12$ & $\rightarrow 6$ & $6.78 \mathrm{E}+11$ & $\rightarrow 4$ & $6.29 \mathrm{E}+11$ & $\rightarrow 32$ & $2.81 \mathrm{E}+11$ & $\rightarrow 143$ & $1.34 \mathrm{E}+11$ & $\rightarrow 5$ & $4.58 \mathrm{E}+12$ \\
\hline 23 & $41 E+11$ & $\rightarrow 43$ & $4.24 \mathrm{E}+11$ & $\rightarrow 150$ & $2.54 \mathrm{E}+11$ & $\rightarrow 42$ & $56 \mathrm{E}+11$ & $\rightarrow 149$ & $5.86 \mathrm{E}+10$ & $\rightarrow 35$ & $1.78 \mathrm{E}+12$ \\
\hline 24 & +11 & $\rightarrow 38$ & 11 & $\rightarrow 147$ & $3.05 \mathrm{E}+11$ & $\rightarrow 41$ & & $\rightarrow 49$ & 9.00 & $\rightarrow 40$ & $E+12$ \\
\hline 325 & $1 E+12$ & $\rightarrow 40$ & $5.91 \mathrm{E}+11$ & $\rightarrow 148$ & $7.23 \mathrm{E}+10$ & $\rightarrow 50$ & & $\rightarrow 56$ & 8.25 & $\rightarrow 174$ & $1.75 \mathrm{E}+12$ \\
\hline 26 & $E+11$ & $\rightarrow 42$ & 4.42 & $\rightarrow 149$ & $1.31 \mathrm{E}+11$ & $\rightarrow 41$ & & & +10 & $\rightarrow 38$ & $1.79 \mathrm{E}+12$ \\
\hline 327 & & $\rightarrow 5$ & & $\rightarrow 33$ & & $\rightarrow 6$ & & 55 & & $\rightarrow 37$ & $8.65 E+11$ \\
\hline 328 & & $\rightarrow 47$ & & $\rightarrow 153$ & & $\rightarrow 35$ & & & & $\rightarrow 46$ & -12 \\
\hline 329 & & $\rightarrow 1$ & & $\rightarrow 164$ & & 52 & & & & $\rightarrow 38$ & \\
\hline 330 & 11 & $\rightarrow 49$ & & $\rightarrow 156$ & 2. & $\rightarrow 41$ & & $\rightarrow 54$ & 10 & $\rightarrow 51$ & 12 \\
\hline 31 & 11 & $\rightarrow 15$ & 1 & $\rightarrow 161$ & 3 & $\rightarrow 158$ & & $\rightarrow$ & 09 & $\rightarrow 160$ & -12 \\
\hline 32 & 12 & $\rightarrow 162$ & 9 & $\rightarrow 97$ & & $\rightarrow 40$ & & $\rightarrow$ & & $\rightarrow 176$ & 12 \\
\hline 33 & $49 \mathrm{E}+11$ & $\rightarrow 161$ & $8.00 \mathrm{E}$ & $\rightarrow 164$ & $6.19 \mathrm{E}+10$ & $\rightarrow 163$ & 1.671 & $\rightarrow 160$ & $2.20 \mathrm{~F}$ & $\rightarrow 165$ & $1.02 \mathrm{E}+12$ \\
\hline 34 & $36 \mathrm{E}+11$ & $\rightarrow 163$ & 0 & $\rightarrow 160$ & $4.09 \mathrm{E}+10$ & $\rightarrow 161$ & $3.78 \mathrm{E}+10$ & $\rightarrow 165$ & $3.90 \mathrm{E}$ & $\rightarrow 151$ & $1.01 \mathrm{E}+12$ \\
\hline 35 & $3 E+11$ & $\rightarrow 164$ & $5.66 \mathrm{E}+10$ & $\rightarrow 162$ & $3.98 \mathrm{E}+10$ & $\rightarrow 160$ & $1.34 \mathrm{E}+09$ & $\rightarrow 152$ & $1.12 \mathrm{E}+09$ & $\rightarrow 155$ & $1.01 \mathrm{E}+12$ \\
\hline 36 & $0 \mathrm{E}+11$ & $\rightarrow 7$ & $1.35 \mathrm{E}+11$ & $\rightarrow 39$ & $1.09 \mathrm{E}+11$ & $\rightarrow 9$ & +10 & $\rightarrow 165$ & 8.83 & $\rightarrow 10$ & $1.21 \mathrm{E}+12$ \\
\hline 37 & $7 E+11$ & $\rightarrow 2$ & $78 \mathrm{E}+11$ & $\rightarrow 24$ & $10 \mathrm{E}+11$ & $\rightarrow 27$ & +11 & $\rightarrow 139$ & -10 & $\rightarrow 145$ & $1.21 \mathrm{E}+12$ \\
\hline 38 & $8 \mathrm{E}+11$ & $\rightarrow 2$ & 11 & $\rightarrow 24$ & 11 & $\rightarrow 139$ & 10 & $\rightarrow 27$ & 10 & $\rightarrow 145$ & $1.06 \mathrm{E}+12$ \\
\hline 39 & & $\rightarrow 24$ & & $\rightarrow 139$ & & $\rightarrow 1$ & & $\rightarrow 2$ & & $\rightarrow 168$ & +11 \\
\hline 340 & & $\rightarrow 2$ & & $\rightarrow 27$ & & $\rightarrow$ & & $\rightarrow 24$ & 10 & $\rightarrow 139$ & $1.93 \mathrm{E}+12$ \\
\hline 341 & & $\rightarrow 2$ & & $\rightarrow 27$ & & $\rightarrow$ & & $\rightarrow$ & & $\rightarrow 15$ & +12 \\
\hline 342 & & $\rightarrow 2$ & & $\rightarrow 27$ & & & & $\rightarrow$ & & $\rightarrow$ & -12 \\
\hline 343 & & $\rightarrow 33$ & & $\rightarrow 155$ & & & & 76 & & $\rightarrow$ & -12 \\
\hline 44 & & $\rightarrow 5$ & & $\rightarrow 37$ & & $\rightarrow$ & & $\rightarrow$ & & $\rightarrow 155$ & 12 \\
\hline 45 & 2 & $\rightarrow 5$ & & $\rightarrow 4$ & & $\rightarrow 37$ & & $\rightarrow 34$ & 11 & $\rightarrow 157$ & 4.3 \\
\hline 46 & 2 & $\rightarrow 4$ & & $\rightarrow 5$ & 1 & $\rightarrow 34$ & & $\rightarrow 36$ & 11 & $\rightarrow 37$ & +12 \\
\hline 47 & $69 \mathrm{E}+12$ & $\rightarrow 4$ & $.74 \mathrm{E}+11$ & $\rightarrow 36$ & $3.46 \mathrm{E}+11$ & $\rightarrow 34$ & $2.05 \mathrm{E}+11$ & $\rightarrow 154$ & $1.20 \mathrm{E}+11$ & $\rightarrow 158$ & $3.91 \mathrm{E}+12$ \\
\hline 48 & $28 \mathrm{E}+12$ & $\rightarrow 7$ & $06 \mathrm{E}-$ & $\rightarrow 10$ & $3.27 \mathrm{E}+11$ & $\rightarrow 36$ & $3.17 \mathrm{E}+11$ & $\rightarrow 5$ & $2.84 \mathrm{E}+11$ & $\rightarrow 6$ & $3.79 \mathrm{E}+12$ \\
\hline 49 & $4 \mathrm{E}+12$ & $\rightarrow 9$ & $.56 \mathrm{E}+11$ & $\rightarrow 10$ & $2.97 \mathrm{E}+11$ & $\rightarrow 44$ & $2.78 \mathrm{E}+11$ & $\rightarrow 8$ & $2.50 \mathrm{E}+11$ & $\rightarrow 7$ & $3.80 \mathrm{E}+12$ \\
\hline 50 & $7 \mathrm{E}+12$ & $\rightarrow 7$ & $11 \mathrm{t}$ & $\rightarrow 39$ & $2.77 \mathrm{E}+11$ & $\rightarrow 165$ & $6.78 \mathrm{E}+10$ & $\rightarrow 37$ & $3.26 \mathrm{E}+10$ & $\rightarrow 157$ & $5.48 \mathrm{E}+12$ \\
\hline 51 & $4 \mathrm{E}+11$ & $\rightarrow 51$ & $.00 \mathrm{E}+11$ & $\rightarrow 169$ & $3.23 \mathrm{E}+11$ & $\rightarrow 50$ & $1.85 \mathrm{E}+11$ & $\rightarrow 168$ & $1.22 \mathrm{E}+11$ & $\rightarrow 49$ & $1.78 \mathrm{E}+12$ \\
\hline 352 & +11 & $\rightarrow 50$ & 1 & $\rightarrow 168$ & $2.31 \mathrm{E}+11$ & $\rightarrow 48$ & 1.14 & $\rightarrow 167$ & +10 & $\rightarrow 40$ & $1.78 \mathrm{E}+12$ \\
\hline 353 & +11 & $\rightarrow 52$ & 1 & $\rightarrow 51$ & $2.71 \mathrm{E}+11$ & $\rightarrow 170$ & 11 & $\rightarrow 169$ & +10 & $\rightarrow 55$ & $1.79 \mathrm{E}+12$ \\
\hline 354 & $2.48 \mathrm{E}+12$ & $\rightarrow 9$ & $5.70 \mathrm{E}+11$ & $\rightarrow 44$ & $3.30 \mathrm{E}+11$ & $\rightarrow 8$ & $2.58 \mathrm{E}+11$ & $\rightarrow 166$ & $2.23 \mathrm{E}+11$ & $\rightarrow 10$ & $4.51 \mathrm{E}+12$ \\
\hline 355 & $1.19 \mathrm{E}+12$ & $\rightarrow 48$ & $6.03 E+11$ & $\rightarrow 167$ & $1.33 \mathrm{E}+08$ & $\rightarrow 155$ & $3.56 \mathrm{E}+07$ & $\rightarrow 33$ & $2.09 \mathrm{E}+07$ & $\rightarrow 343$ & $1.79 \mathrm{E}+12$ \\
\hline 356 & +11 & $\rightarrow 54$ & 11 & $\rightarrow 172$ & +11 & $\rightarrow 49$ & $1.36 \mathrm{E}+11$ & $\rightarrow 56$ & $8.49 \mathrm{E}+10$ & $\rightarrow 174$ & $1.76 \mathrm{E}+12$ \\
\hline 357 & $E+11$ & $\rightarrow 52$ & $3.44 \mathrm{E}+11$ & $\rightarrow 53$ & $3.35 \mathrm{E}+11$ & $\rightarrow 170$ & $1.82 \mathrm{E}+11$ & $\rightarrow 171$ & $1.23 \mathrm{E}+11$ & $\rightarrow 51$ & $1.80 \mathrm{E}+12$ \\
\hline 58 & +11 & $\rightarrow 53$ & $4.72 \mathrm{E}+11$ & $\rightarrow 171$ & +11 & $\rightarrow 52$ & $1.33 \mathrm{E}+11$ & $\rightarrow 170$ & $4.14 \mathrm{E}$ & $\rightarrow 19$ & $1.81 \mathrm{E}+12$ \\
\hline 59 & $E+11$ & $\rightarrow 175$ & $1.01 \mathrm{E}+11$ & $\rightarrow 178$ & 1.0 & $\rightarrow 176$ & 5.03 & $\rightarrow 1$ & $3.29 \mathrm{E}+09$ & $\rightarrow 179$ & $1.01 \mathrm{E}+12$ \\
\hline 360 & $9.46 \mathrm{E}+11$ & $\rightarrow 56$ & $5.83 \mathrm{E}+11$ & $\rightarrow 174$ & $8.66 \mathrm{E}+10$ & $\rightarrow 40$ & $1.08 \mathrm{E}+10$ & $\rightarrow 148$ & $9.57 \mathrm{E}+09$ & $\rightarrow 66$ & $1.65 \mathrm{E}+12$ \\
\hline
\end{tabular}


Table 5. continued.

\begin{tabular}{|c|c|c|c|c|c|c|c|c|c|c|c|}
\hline Level & $r\left(\mathrm{~s}^{-1}\right)$ & $\begin{array}{l}\text { final } \\
\text { level }\end{array}$ & $r\left(\mathrm{~s}^{-1}\right)$ & $\begin{array}{l}\text { final } \\
\text { level }\end{array}$ & $A^{r}\left(\mathrm{~s}^{-1}\right)$ & $\begin{array}{l}\text { final } \\
\text { level }\end{array}$ & $A^{r}\left(\mathrm{~s}^{-1}\right)$ & $\begin{array}{l}\text { final } \\
\text { level }\end{array}$ & $A^{r}\left(\mathrm{~s}^{-1}\right)$ & $\begin{array}{l}\text { final } \\
\text { level }\end{array}$ & $\sum A^{r}\left(\mathrm{~s}^{-1}\right)$ \\
\hline 61 & $74 \mathrm{E}+11$ & $\rightarrow 55$ & $96 \mathrm{E}+11$ & $\rightarrow 173$ & $23 \mathrm{E}+11$ & $\rightarrow 54$ & $1.61 \mathrm{E}+11$ & $\rightarrow 172$ & $.13 \mathrm{E}+11$ & $\rightarrow 47$ & $.78 \mathrm{E}+12$ \\
\hline 52 & $E+11$ & 177 & $26 \mathrm{E}+11$ & $\rightarrow 175$ & $5 E+10$ & $\rightarrow 183$ & $1.01 \mathrm{E}+10$ & $\rightarrow 176$ & $40 \mathrm{E}+09$ & $\rightarrow 182$ & $1.02 \mathrm{E}+12$ \\
\hline 3 & $78 \mathrm{E}+11$ & $\rightarrow 176$ & $15 E+11$ & $\rightarrow 179$ & $4 E+10$ & $\rightarrow 182$ & $1.27 \mathrm{E}+09$ & $\rightarrow 162$ & $.57 \mathrm{E}+08$ & $\rightarrow 50$ & $.01 \mathrm{E}+12$ \\
\hline 54 & $95 \mathrm{E}+11$ & $\rightarrow 178$ & & $\rightarrow 176$ & $33 E+10$ & $\rightarrow 175$ & $3.67 \mathrm{E}+10$ & $\rightarrow 182$ & $.01 E+09$ & $\rightarrow 179$ & $1.02 \mathrm{E}+12$ \\
\hline 55 & $76 \mathrm{E}+11$ & $\rightarrow 182$ & $.22 \mathrm{E}+10$ & $\rightarrow 176$ & $.96 \mathrm{E}+09$ & $\rightarrow 179$ & $1.14 \mathrm{E}+09$ & $\rightarrow 56$ & $9.68 \mathrm{E}+08$ & $\rightarrow 162$ & $.94 \mathrm{E}+11$ \\
\hline 66 & $46 \mathrm{E}+11$ & $\rightarrow 181$ & $83 E+11$ & $\rightarrow 177$ & $.12 \mathrm{E}+10$ & $\rightarrow 185$ & $2.30 \mathrm{E}+10$ & $\rightarrow 175$ & $.37 E+09$ & $\rightarrow 183$ & $.02 \mathrm{E}+12$ \\
\hline 67 & $95 \mathrm{E}+11$ & $\rightarrow 179$ & $33 \mathrm{E}+09$ & $\rightarrow 48$ & $34 \mathrm{E}+08$ & $\rightarrow 167$ & $9.22 \mathrm{E}+05$ & $\rightarrow 33$ & $89 \mathrm{E}+04$ & $\rightarrow 355$ & $.97 \mathrm{E}+11$ \\
\hline 68 & $78 \mathrm{E}+11$ & $\rightarrow 183$ & $69 E+11$ & $\rightarrow 178$ & $25 E+10$ & $\rightarrow 175$ & $2.07 \mathrm{E}+10$ & $\rightarrow 177$ & $.52 E+09$ & $\rightarrow 176$ & $.02 \mathrm{E}+12$ \\
\hline 69 & 11 & $\rightarrow 184$ & & $\rightarrow 181$ & $5 \mathrm{E}+10$ & $\rightarrow 177$ & 2.59 & $\rightarrow 185$ & $6 E+09$ & $\rightarrow 183$ & $01 \mathrm{E}+12$ \\
\hline 70 & & 185 & & $\rightarrow 183$ & & $\rightarrow 181$ & 09 & $\rightarrow 178$ & +09 & $\rightarrow 177$ & \\
\hline 71 & & 59 & & $\rightarrow 180$ & & $\rightarrow 55$ & & $\rightarrow 173$ & & $\rightarrow 47$ & -12 \\
\hline 2 & & $\rightarrow 2$ & & $\rightarrow 1$ & & $\rightarrow 18$ & & $\rightarrow 128$ & & $\rightarrow 267$ & -11 \\
\hline 3 & & 16 & & $\rightarrow 126$ & & $\rightarrow 260$ & 4.2 & $\rightarrow 20$ & & $\rightarrow 129$ & -11 \\
\hline 4 & & 16 & & $\rightarrow 126$ & & $\rightarrow 260$ & & $\rightarrow 21$ & & $\rightarrow 130$ & 11 \\
\hline 15 & & 1 & & $\rightarrow 17$ & & $\rightarrow 2$ & 1.7 & $\rightarrow 127$ & & $\rightarrow 266$ & 12 \\
\hline 6 & +12 & $\rightarrow 2$ & 11 & $\rightarrow 18$ & 11 & $\rightarrow 128$ & 9.97 & $\rightarrow 267$ & +10 & $\rightarrow 27$ & $2.47 \mathrm{E}+12$ \\
\hline 7 & & 20 & 11 & $\rightarrow 129$ & -11 & $\rightarrow 270$ & 3.71 & $\rightarrow 21$ & +10 & $\rightarrow 130$ & $1.05 \mathrm{E}+12$ \\
\hline 78 & 11 & $\rightarrow 21$ & & $\rightarrow 130$ & & $\rightarrow 272$ & 4.3 & $\rightarrow 60$ & +09 & $\rightarrow 2$ & $1.05 \mathrm{E}+12$ \\
\hline 79 & $56 \mathrm{E}+11$ & $\rightarrow 279$ & & $\rightarrow 201$ & & $\rightarrow 280$ & & $\rightarrow 202$ & & $\rightarrow 131$ & -11 \\
\hline 30 & -11 & 31 & & $\rightarrow 277$ & & 32 & & $\rightarrow 278$ & & $\rightarrow 67$ & +11 \\
\hline 81 & & $\rightarrow 280$ & & $\rightarrow 202$ & & $\rightarrow 132$ & 2.6 & $\rightarrow 278$ & -07 & $\rightarrow 114$ & $0 \mathrm{E}+11$ \\
\hline 32 & & $\rightarrow 132$ & & $\rightarrow 278$ & & $\rightarrow 66$ & & $\rightarrow 195$ & & $\rightarrow 21$ & +11 \\
\hline 33 & & $\rightarrow 9$ & & $\rightarrow 61$ & & $\rightarrow 6$ & & $\rightarrow 10$ & & $\rightarrow 191$ & +12 \\
\hline 34 & & $\rightarrow 10$ & & $\rightarrow 60$ & & $\rightarrow 190$ & & $\rightarrow 7$ & & $\rightarrow 58$ & \\
\hline 35 & & $\rightarrow 2$ & & $\rightarrow 46$ & & $\rightarrow 45$ & & $\rightarrow 1$ & & $\rightarrow 187$ & \\
\hline 36 & & $\rightarrow 2$ & & $\rightarrow 46$ & & $\rightarrow 45$ & & $\rightarrow 1$ & & $\rightarrow 187$ & \\
\hline 37 & & $\rightarrow 2$ & & $\rightarrow 46$ & & $\rightarrow 187$ & & $\rightarrow 195$ & & $\rightarrow 66$ & \\
\hline 38 & & $\rightarrow 2$ & & $\rightarrow 45$ & & $\rightarrow 1$ & & $\rightarrow 46$ & & $\rightarrow 186$ & \\
\hline 39 & & $\rightarrow 2$ & & $\rightarrow 1$ & & $\rightarrow 45$ & & $\rightarrow 46$ & & $\rightarrow 186$ & 12 \\
\hline 00 & 12 & $\rightarrow 8$ & & $\rightarrow 9$ & & $\rightarrow 6$ & 4.4 & $\rightarrow 57$ & -11 & $\rightarrow 10$ & $4.77 E+12$ \\
\hline 91 & & $\rightarrow 6$ & & $\rightarrow 58$ & & $\rightarrow 188$ & 1.9 & $\rightarrow 7$ & -11 & $\rightarrow 60$ & $3.38 \mathrm{E}+12$ \\
\hline 92 & & $\rightarrow 7$ & & $\rightarrow 60$ & & $\rightarrow 190$ & & $\rightarrow 272$ & 10 & $\rightarrow 80$ & +12 \\
\hline 93 & -12 & $\rightarrow 10$ & $6.44 \mathrm{E}+11$ & $\rightarrow 61$ & $80 \mathrm{E}+11$ & $\rightarrow 191$ & $1.58 \mathrm{E}+11$ & $\rightarrow 7$ & +11 & $\rightarrow 6$ & $4.80 \mathrm{E}+12$ \\
\hline 94 & & $\rightarrow 8$ & & $\rightarrow 10$ & & $\rightarrow 57$ & & $\rightarrow 189$ & -11 & $\rightarrow 62$ & $3.98 \mathrm{E}+12$ \\
\hline 95 & -12 & $\rightarrow 10$ & & $\rightarrow 9$ & 11 & $\rightarrow 63$ & 2.0 & $\rightarrow 192$ & -11 & $\rightarrow 61$ & $3.97 \mathrm{E}+12$ \\
\hline 96 & & $\rightarrow 69$ & & $\rightarrow 193$ & & $\rightarrow 67$ & & $\rightarrow 194$ & & $\rightarrow 71$ & +12 \\
\hline 97 & & $\rightarrow 71$ & & $\rightarrow 196$ & & $\rightarrow 66$ & & $\rightarrow 195$ & & $\rightarrow 54$ & $1.79 \mathrm{E}+12$ \\
\hline 398 & & $\rightarrow 199$ & & $\rightarrow 201$ & & $\rightarrow 200$ & & $\rightarrow 279$ & & $\rightarrow 202$ & $1.04 \mathrm{E}+12$ \\
\hline 99 & & $\rightarrow 67$ & & $\rightarrow 194$ & & $\rightarrow 66$ & & $\rightarrow 277$ & & $\rightarrow 196$ & +12 \\
\hline 00 & & 200 & & $\rightarrow 202$ & & $\rightarrow 280$ & & $\rightarrow 201$ & & $\rightarrow 71$ & $04 E+12$ \\
\hline 1 & & $\rightarrow 66$ & & $\rightarrow 195$ & & $\rightarrow 278$ & & $\rightarrow 56$ & & $\rightarrow 100$ & \\
\hline 2 & & $\rightarrow 73$ & & $\rightarrow 197$ & & $\rightarrow 69$ & & $\rightarrow 74$ & & $\rightarrow 193$ & $.82 \mathrm{E}+12$ \\
\hline 3 & & $\rightarrow 74$ & & $\rightarrow 198$ & & $\rightarrow 71$ & 1.58 & $\rightarrow 75$ & & $\rightarrow 196$ & $1.81 \mathrm{E}+12$ \\
\hline & & $\rightarrow 201$ & & $\rightarrow 279$ & & $\rightarrow 202$ & & $\rightarrow 280$ & -09 & $\rightarrow 67$ & $1.02 \mathrm{E}+12$ \\
\hline & & $\rightarrow 202$ & & $\rightarrow 2$ & & $\rightarrow 66$ & 6.71 & $\rightarrow 114$ & 08 & $\rightarrow 271$ & $.02 \mathrm{E}+12$ \\
\hline 06 & & $\rightarrow 20$ & & $\rightarrow 1$ & & $\rightarrow 2 \mathrm{C}$ & & $\rightarrow 279$ & 09 & $\rightarrow 200$ & $.04 \mathrm{E}+12$ \\
\hline 407 & 11 & $\rightarrow 204$ & $8.11 \mathrm{H}$ & $\rightarrow 200$ & $2.02 \mathrm{~B}$ & $\rightarrow 280$ & $3.93 \mathrm{E}+09$ & $\rightarrow 199$ & $2.07 \mathrm{E}+09$ & $\rightarrow 124$ & $1.04 \mathrm{E}+12$ \\
\hline
\end{tabular}

